\author{
by \\ Joe C. Turnege,Allan F. Henry \\ March 1972
}

\begin{abstract}
Massachusetts Institute of Technology
Department of Nuclear Engineering

Cambridge, Massachusetts 02139
\end{abstract}

\author{
AEC Research and Development Report \\ Contract AT(11-1)-3052 \\ U.S. Atomic Energy Commission
}




\section{DISCLAIMER}

This report was prepared as an account of work sponsored by an agency of the United States Government. Neither the United States Government nor any agency Thereof, nor any of their employees, makes any warranty, express or implied, or assumes any legal liability or responsibility for the accuracy, completeness, or usefulness of any information, apparatus, product, or process disclosed, or represents that its use would not infringe privately owned rights. Reference herein to any specific commercial product, process, or service by trade name, trademark, manufacturer, or otherwise does not necessarily constitute or imply its endorsement, recommendation, or favoring by the United States Government or any agency thereof. The views and opinions of authors expressed herein do not necessarily state or reflect those of the United States Government or any agency thereof. 


\section{DISCLAIMER}

Portions of this document may be illegible in electronic image products. Images are produced from the best available original document. 


\title{
A NEW APPROACH TO SOLVING THE
}

\author{
MULTIMODE KINETICS EQUATIONS
}

by

Joe C. Turnage

Submitted to the Department of Nuclear Engineering on February 24, 1972 in partial fulfillment of the requirements for the degree of Doctor of Philosophy.

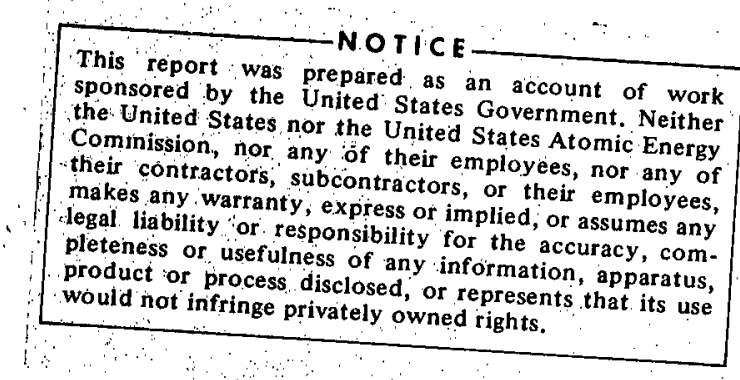

\section{ABSTRACT}

An investigation is made of a new approach to solving a set of equations (the multimode kinetics equations) which have been obtained by the application of time synthesis to the multigroup neutron diffusion equations. The multimode kinetics equations are cast into the form of the point kinetic equations and successfully solved utilizing the application of an analytic inversion of the matrix $[I-\delta A]$ to both the Padé $(2,0)$ and "modified" Crank-Nicholson approximations to exp[st[A]]. 
THIS PAGE

\section{WAS INTENTIONALLY LEFT BLANK}


TABLE OF CONTENTS

Page

Abstract 2

List of Tables $\quad 5$

List of Figures 6

$\begin{array}{ll}\text { Acknowledgments } & 7\end{array}$

$\begin{array}{ll}\text { Biographical Note . } & 8\end{array}$

Chapter I. INTRODUCTION 9 9

1.1 The Use of Space-Dependent Kinetics 9

1.2 Some Current Methods of Solving the Time-Dependent Multigroup Diffusion Equation 11

1.3 A Development of the Multimode Kinetics Equations 35

Chapter II. A NEW APPROACH TO SOLVING THE MULTIMODE KINETICS EQUUATIONS

2. 1 A Review of Techniques for Solving Multimode Kinetics Equations Which Have Been Cast in 'a Point-Kinetics Form

2. 2 An Alternate Approach to the Solution of the Multimode Kinetics Equations - The Approximation of $\exp (\Delta t[A])$

2. 3 Development of an Analytic Inversion of $[[\mathrm{I}]-\delta[\mathrm{A}]]$

2. 4 Application of the Analytic Inversion to $\left[\psi_{p+1}\right]=$ $\mathrm{E}_{2.0}(\Delta t[\mathrm{~A}])\left[\psi_{\mathrm{p}}\right]$

2. 5 Application of the Analytic Inversion to $\left[\psi_{p+1}\right]=$ $E_{1,1}^{\prime}(\Delta t[A])\left[\psi_{p}\right]$

Chapter III. NUMERICAL RESULTS

3.1 Reactor Number One - One-Group Results

3. 2 Reactor Number Two - Two-Group Results 
Chapter IV. CONCLUSIONS AND RECOMMENDATIONS 92

4. 1 Conclusions $\quad 92$

4. 2 Recommendations for Further Work _ _ . 93

REFERENCES 95

Appendix I Proof of $[e]=\left[a b^{-1} a+b\right]^{-1}$ and $[z]=\left[b^{-1} a e\right]=$ $\left[\mathrm{ba}^{-1} \mathrm{~b}+\mathrm{a}\right]^{-1} \quad 98$

$\begin{array}{ll}\text { Appendix II Proof of Identities } & 100\end{array}$

Appendix III An Example of How [REAL] May Be Structured Using the Identities $(2.34)$ and $(2.35)$

Appendix IV Description of Computer Codes 106 


\section{LIST OF TABLES}

Table III-1. Parameters for Criticality

Table III-2. Comparison of Amplitude Functions

Table III-3. Comparison with $\theta$-Method

Table III-4. Comparison of Amplitude Functions

Table III-5. Comparison with $\theta$-Method

Table III-6. Comparison of Amplitude Functions

Table III-7. Comparison with $\theta-$ Method

Table III-8. Parameters for Criticality

Table III-9. Delayed Neutron Parameters

Table III-10. Comparison of Amplitude Functions

Table III-11. Comparison with GAKIN Method 


\section{LIST OF FIGURES}

Figure III-1. Trial and Weight Functions, Case 1 72

Figure III-2. Trial and Weight Functions, Case 2 77

Figure III-3. Trial and Weight Functions, Case 3

Figure III-4. Trial and Weight Function \# 1, Two-Group Problem

Figure III-5. Trial and Weight Function \#2, Two-Group Problem 


\section{Acknowledgments}

There are several people and organizations who deserve the author's deep appreciation for their encouragement and advice over the course of this work.

The author is particularly grateful for the guidance and friendship given to him by Professors Allan F. Henry and Kent F. Hansen. Dr. J. A. W. daNóbrega also provided helpful advice on several occasions.

The author's wife, Ellen, should be acknowledged for her support and commended for her tolerance over the last four and one-half years. And his parents, Clayton and Ollion Turnage, deserve acknowledgment for having provided so many years of guidance and devotion.

Fellowship support for the author was provided by the U.S. Atomic Energy Commission. The work was performed under USAEC Contract $A T(11-1)-3052$. All computation was performed on either an IBM $360 / 65$ or $370 / 155$ at the M. I. T. Information Processing Center. 


\section{Biographical Note}

Joe Clayton Turnage was born on November 19, 1945, in Tucson, Arizona. Shortly thereafter his family moved to Jackson, Mississippi, where he later attended elementary and secondary school.

He enrolled at Mississippi State University in September, 1963, and during the next four years was active in collegiate debating and campus political activity. While an undergraduate he served as an officer in Kappa Alpha Order social fraternity, and as Attorney General of the Student Association. He was elected to Blue Key Nation Honor Society, Tau Beta Pi, and Phi Kappa Phi and was selected to appear in Who's Who in American Colleges and Universities. In June, 1967, he was graduated with Distinction with a Bachelor of Science degree in Nuclear Engineering.

In September, 1967, he entered the Massachusetts Institute of Technology as a student in the Department of Nuclear Engineering with an O. R. A. U. Special Fellowship in Nuclear Science on Engineering. He received a Master of Science degree in Nuclear Engineering in February, 1970.

Mr. Turnage is married to the former Ruth Ellen Ezelle of Forrest, Mississippi. They have one son, Clayton Jonathan. 


\section{CHAPTER I}

\section{IN TRODUC TION}

\subsection{The Use of Space-Dependent Kinetics}

Although the essential problem confronting the reactor physicist has remained the same since the early 1940's, the emphasis of the physicist's approach has shifted considerably. Stated simply, the problem is: How is one to predict the behavior of a neutron population in a material medium? It was this problem that Fermi faced in June of 1942, when he derived a value of 1.07 for the infinite medium multiplication factor for a uranium oxide-graphite lattice. And it is roughly the same problem that today's reactor physicist must tackle. The approach to the problem over the past few years, however, has been focused in large part on the desirability of knowing as much as possible about when and where heat is produced in a power reactor. Thus (as S. Kaplan pointed out in 1966) it is extremely important to be able "to predict what the spatial distribution of the fission rate will be at all times during the life of the reactor, under all static and dynamic operating conditions, and during various postulated accidental transients." ${ }^{1}$

The early experiments of Fermi at Columbia University in 1942 were designed to investigate the possibility of a neutron chain reaction. Later that year, at Chicago, confirmation of the chain reaction was obtained and the nuclear energy business was born. Today that business is concerned with the design and construction of large $(\geqslant 1,000$ $M_{w(e)}$ thermal reactors, and with the development of fast breeder reactors. 
The prediction of the spatial distribution of the fission rate for these devices has involved, over the past few years, the development of methods for solving the time-dependent, multigroup diffusion equation (and various approximations to it) in one or more spatial dimensions. This approach to the problem of predicting the behavior of a neutron population in a material medium (a substantially different approach from that of Fermi) has evolved partly because of the recognition that in the large, loosely-coupled thermal reactors being designed today the neutron population will not exhibit a spatially uniform behavior in response to a localized perturbation. In fast breeder development, this approach is viewed as part of a necessarily strong emphasis on safety.

The degree of sophistication with which one tackles the solution of the time-dependent, multigroup neutron diffusion equation is usually dictated by the kind of event being considered, the level of sophistication of the computational machinery at the reactor physicist's disposal, and the price the reactor physicist is willing to pay. For a given reactor, both the nonuniformity of the spatial redistribution of the neutron population following a perturbation and the time scale of the redistribution depend on the type of perturbation which has occurred. Consequently, the methodology that one chooses to model the neutronics of the reactor should be based in large part on one's knowledge of the event, given the constraints of computational cost and accuracy that then select the "best" method from a list of comparable methods.

This thesis is concerned with the analysis of a new approach to solving a set of equations (the multimode kinetics equations) which 
cheaply approximate the predictions of the time-dependent, multigroup neutron diffusion equation, along with the associated set of timedependent neutron precursor equations. The remainder of this chapter involves a brief review of several of the current techniques for approximating such predictions, and a development of the point kinetics form of the multimode kinetics equations using a time-synthesis approximation. Chapter II begins with an analysis of the current approach to solving these equations and goes on to develop a new approach, based on the Padé $(2,0)$ and a modified Padé $(1,1)$ approximation to the exponential. Some numerical results which test the new approach are given in Chapter III. And, finally, both the conclusions concerning the worth of the new approach and some recommendations for further work are found in Chapter IV.

\subsection{Some Current Methods of Solving the Time-Dependent,}

Multigroup Diffusion Equation

Before a development of the multimode kinetics equations is begun, it would seem wise to consider the equations they approximate. The time-dependent, external-source-free neutron diffusion equation for energy group g, along with the associated equation needed to determine the concentration of delayed neutron precursors belonging to delayed precursor group i may be written as ${ }^{2}$

$$
\begin{aligned}
\vec{\nabla} \cdot D_{g}(\vec{r}, t) & \vec{\nabla} \phi_{g}(\vec{r}, t)-\sum_{t g}(\vec{r}, t) \phi_{g}(\vec{r}, t)+\sum_{j} x_{p g}^{j}\left(1-\beta^{j}\right) \sum_{g^{\prime}} v^{j} \Sigma_{f g^{\prime}}(\vec{r}, t) \phi_{g^{\prime}}(\vec{r}, t) \\
& +\sum_{g^{\prime}} \Sigma_{s g g^{\prime}}(\vec{r}, t) \phi_{g^{\prime}}(\vec{r}, t)+\sum_{i} x_{i g^{\prime}} \lambda_{i} C_{i}(\vec{r}, t)=\frac{\partial}{\partial t}\left(\frac{\phi_{g}(\vec{r}, t)}{v_{g}}\right)
\end{aligned}
$$




$$
\sum_{j} \beta_{i}^{j} \sum_{g^{\prime}} v^{j} \sum_{f g^{\prime}}(\vec{r}, t) \phi_{g^{\prime}}(\vec{r}, t)-\lambda_{-i} C_{i}(\vec{r}, t)=\frac{\partial}{\partial t}\left(C_{i}(\vec{r}, t)\right) \quad i=1,2, \ldots I \quad(1,2)
$$

where the superscript $\mathrm{j}$ refers to the $\mathrm{j}^{\text {th }}$ fissionable isotope.

In equations $(1,1)$ and $(1.2)$ :

(a) $\vec{\nabla} \cdot D_{g}(\vec{r}, t) \vec{\nabla} \phi_{g}(\vec{r}, t)$ is the net leakage rate per unit volume of neutrons belonging to energy group $g$ at location $\vec{r}$ and time $t$.

(b) $\Sigma_{\operatorname{tg}}(\vec{r}, t) \phi_{g}(\vec{r}, t)$ is the total neutron reaction rate per unit volume for neutrons belonging to energy group $g$ at location $\vec{r}$ and time $t$.

(c) $\sum_{j} x_{p g}^{j}\left(1-\beta^{j}\right) \sum_{g^{\prime}} v^{j} \Sigma_{f g^{\prime}}(\vec{r}, t) \phi_{g^{\prime}}(\vec{r}, t)$ is the production rate per unit volume of prompt neutrons belonging to energy group $g$ at location $\vec{r}$ and time $t$.

(d) $\sum_{i} x_{i g} \lambda_{i} C_{i}(\vec{r}, t)$ is the production rate per unit volume, resulting from the decay of delayed emitters, of neutrons belonging to energy group $g$ at location $\vec{r}$ and time $t$.

(e) $\sum_{g^{\prime}} \Sigma_{s g^{\prime}}(\vec{r}, t) \phi_{g^{\prime}}(\vec{r}, t)$ is the production rate per unit volume, due to scattering, of neutrons belonging to energy group $g$ at location $\vec{r}$ and time $t$.

(f) $\frac{\partial}{\partial t}\left(\frac{\phi_{g}(\vec{r}, t)}{v_{g}}\right)$ is the rate of increase per unit volume of the number of neutrons belonging to energy group $g$ at location $\vec{r}$ and time $t$. 
(g) $\sum_{j} \beta_{i}^{j} \sum_{g^{\prime}} v^{j} \Sigma_{f g^{\prime}}^{j}(\vec{r}, t) \phi_{g}^{\prime}(\vec{r}, t)$ is the production rate per unit volume of delayed emitters of type $i$ at location $\vec{r}$ and time $t$.

(h) $\lambda_{i} C_{i}(\vec{r}, t)$ is the rate of decay per unit volume of delayed emitters of type $i$ at location $\vec{r}$ and time $t$.

(i) $\frac{\partial}{\partial t}\left(C_{i}(\vec{r}, t)\right)$ is the time rate of change per unit volume of delayed emitters of type $i$ at location $\vec{r}$ and time $t$.

For G groups and I precursors, equations (1.1) and (1.2) may be written in matrix form as follows:

$$
\begin{aligned}
& \vec{\nabla} \cdot\left[\begin{array}{ccc}
D_{1}(\vec{r}, t) & & \\
& \ddots & \\
& & D_{G}(\vec{r}, t)
\end{array}\right] \vec{\nabla}\left[\begin{array}{c}
\phi_{1}(\vec{r}, t) \\
\vdots \\
\phi_{G}(\vec{r}, t)
\end{array}\right] \\
& +\sum_{j}\left(1-\beta^{j}\right)\left[\begin{array}{c}
x_{p l}^{j} \\
\vdots \\
x_{p G}^{j}
\end{array}\right]\left[v_{\Sigma_{f l}^{j}(\vec{r}, t) \ldots v^{j} \Sigma_{f G}^{j}(\vec{r}, t)}^{j^{j}}\right]\left[\begin{array}{c}
\phi_{1}(\vec{r}, t) \\
\vdots \\
\phi_{G}(\vec{r}, t)
\end{array}\right] \\
& -\left[\begin{array}{cccc}
\Sigma_{t, 1}(\vec{r}, t)-\Sigma_{s 11}(\vec{r}, t) & -\Sigma_{s 12}(\vec{r}, t) & \cdots & -\Sigma_{s 1 G}(\vec{r}, t) \\
-\Sigma_{s 21}(\vec{r}, t) & \Sigma_{t 2}(\vec{r}, t)-\Sigma_{s 22^{(\vec{r}, t)}} & \cdots & -\Sigma_{s 2 G}(\vec{r}, t) \\
\vdots & \cdots & \vdots \\
-\Sigma_{s G 1}(\vec{r}, t) & & & \Sigma_{t G}(\vec{r}, t)-\Sigma_{s G G}(\vec{r}, t)
\end{array}\right] \\
& \cdot\left[\begin{array}{c}
\phi_{1}(\vec{r}, t) \\
\vdots \\
\phi_{G}(\vec{r}, t)
\end{array}\right]+\sum_{i}^{I} \lambda_{i} C_{i}(\vec{r}, t)\left[\begin{array}{c}
x_{i l} \\
\vdots \\
x_{i G}
\end{array}\right]=\frac{\partial}{\partial t}\left[\begin{array}{cc}
\frac{1}{v_{1}} & \\
& \ddots \\
0 & \frac{1}{v_{G}}
\end{array}\right]\left[\begin{array}{c}
\phi_{1}(\vec{r}, t) \\
\vdots \\
\phi_{G}(\vec{r}, t)
\end{array}\right]
\end{aligned}
$$




$$
\begin{aligned}
& \sum_{j} \beta_{i}^{j}\left[\nu^{j} \sum_{f l}^{j}(\vec{r}, t) \ldots v^{j} \Sigma_{f G}^{j}(\vec{r}, t)\right]\left[\begin{array}{c}
\phi_{1}(\vec{r}, t) \\
\vdots \\
\phi_{G}(\vec{r}, t)
\end{array}\right]-\lambda_{i} C_{i}(\vec{r}, t)=\frac{\partial C_{i}(\vec{r}, t)}{\partial t} \\
& i=1,2, \ldots I .
\end{aligned}
$$

Equations (1.3) and (1.4) may now be written as

$$
\begin{aligned}
\vec{\nabla} \cdot[D(\vec{r}, t)] \vec{\nabla}[\Phi(\vec{r}, t)]+\sum_{j}\left(1-\beta^{j}\right)\left[x_{\rho}^{j}\right]\left[\nu^{j} \Sigma_{f}^{j}(\vec{r}, t)\right]^{T}[\Phi(\vec{r}, t)] \\
-[A(\vec{r}, t)][\Phi(\vec{r}, t)]+\sum_{i=1}^{I} \lambda_{i} C_{i}(\vec{r}, t)\left[x_{i}\right]=\frac{\partial}{\partial t}[v]^{-1}[\Phi(\vec{r}, t)] \\
\sum_{j} \beta_{i}^{j}\left[\nu^{j} \Sigma_{f}^{j}(\vec{r}, t)\right]^{T}[\Phi(\vec{r}, t)]-\lambda_{i} C_{i}(\vec{r}, t)=\frac{\partial C_{i}(\vec{r}, t)}{\partial t} \quad i=1,2, \ldots I .
\end{aligned}
$$

For assemblies the size of current power reactors, it is generally assumed that equations (1.5) and (1.6) are sufficient to describe the spatial and energy distribution of the neutron flux in the reactor as a function of time. Having adopted this position, one must decide how to go about solving the equations.

Certain parallelisms in structure exist in the development of two general approaches to solving these equations. Since analytic solutions of equations (1.5) and (1.6) can be obtained only in the most trivial cases, direct approaches involve finite differencing the equations in both space and time. Indirect approaches include those classes of approximations generally referred to as modal methods and spacetime synthesis methods. Typically, indirect methods involve an attempt to reduce the number of independent variables at hand. They 
therefore require one to recombine trial functions and coefficients to describe the neutron flux distribution.

In general, one may divide those direct techniques which depend upon finite differencing in space and time into at least three general categories. All involve the replacement of all the time derivatives and the Laplacian term of equations $(1.5)$ and $(1.6)$ by their finite difference counterparts. This transformation results in a set of equations which are sufficient to describe the energy group fluxes and the precursor. group concentrations at each spatial mesh point as a function of time.

To recast equations $(1.5)$ and $(1.6)$ into a finite-differenced form in a direct manner, one typically begins by forming a set of semidiscrete equations. This is done by superimposing a three-dimensional spatial mesh over the reactor of interest, integrating the resulting, spatially discretized form of equations (1.5) and (1.6) over the volumes associated with each of the mesh points, and assuming that the neutron current may be approximated by a finite difference relationship. The resulting equations for the neutron flux at all mesh points for energy group $\mathrm{g}$ and for the $\mathrm{i}^{\text {th }}$ delayed neutron precursor group may be written as 3

$$
\frac{d}{d t}\left[\psi_{g}\right]=\left[D_{g}\right]\left[\psi_{g}\right]+\sum_{i=1}^{I}\left[F_{g i}\right]\left[C_{i}\right]+\sum_{g^{\prime}=1}^{G}\left[T_{g^{\prime}}\right]\left[\psi_{g^{\prime}}\right]
$$

and

$$
\frac{\mathrm{d}}{\mathrm{dt}}\left[\mathrm{C}_{i}\right]=-\left[\Lambda_{i}\right]\left[\mathrm{C}_{i}\right]+\sum_{\mathrm{g}^{\prime}=1}^{\mathrm{G}}\left[\mathrm{P}_{\mathrm{ig}}\right]\left[\psi_{\mathrm{g}^{\prime}}\right]
$$


In equations $(1.7)$ and $(1.8)$ :

(a) $\left[D_{g}\right]\left[\psi_{g}\right]$ is the finite-differenced, box-integrated counterpart of the operation $v_{g}\left\{\vec{\nabla} \cdot D_{g}(\vec{r}, t) \vec{\nabla} \phi_{g}(\vec{r}, t)\right\} \cdot\left[D_{g}\right]$ is a seven-stripe matrix representing the process of neutron leakage across the six sides of the mesh volume.

(b) $\sum_{g^{\prime}=1}^{G}\left[T_{g g^{\prime}}\right]\left[\psi_{g^{\prime}}\right]$ is the finite-differenced, box-integrated counterpart of $v_{g}\left\{\sum_{j} x_{p g^{j}}\left(1-\beta^{j}\right) \sum_{g^{\prime}} v^{j} \Sigma_{f g^{\prime}}(\vec{r}, t) \phi_{g^{\prime}}(\vec{r}, t)+\sum_{g^{\prime}} \Sigma_{s g^{\prime}}(\vec{r}, t) \phi_{g^{\prime}}(\vec{r}, t)-\right.$ $\left.\Sigma_{\operatorname{tg}}(\vec{r}, t) \phi_{g}(\vec{r}, t)\right\} \cdot\left[T_{g g^{\prime}}\right]$ contains terms representing absorption and intergroup scattering processes.

(c) $\left[\mathrm{F}_{\mathrm{gi}}\right]\left[\mathrm{C}_{\mathrm{i}}\right]$ is the finite-differenced, box-integrated counterpart of $v_{g} x_{i g} \lambda_{i} C_{i}(\vec{r}, t)$. The matrix product $\left[F_{g i}\right]\left[C_{i}\right]$ concerns the transfer of delayed neutrons into group $g$ due to decays in precursor group $i$.

(d) $\left[\Lambda_{i}\right]\left[\mathrm{C}_{\mathbf{i}}\right]$ is the finite-differenced, box-integrated counterpart to $\lambda_{i} C_{i}(\vec{r}, t)$. The matrix $\left[\Lambda_{i}\right]$ contains the precursor decay constants. (e) $\sum_{g^{\prime}=1}^{G}\left[P_{i g^{\prime}}\right]\left[\psi_{g^{\prime}}\right]$ is the finite-differenced, box-integrated counterpart

to $\sum_{j} \beta_{i}^{j} \sum_{g^{\prime}} v^{j} \Sigma_{f g^{\prime}}(\vec{r}, t) \phi_{g^{\prime}}(\vec{r}, t)$ and represents the production rate of the $i^{\text {th }}$ delayed precursor due to fissions in group $g^{\prime}$.

The semi-discrete equations (1.7) and (1.8) may be combined into the single matrix equation

$$
\frac{\mathrm{d}}{\mathrm{dt}}[\Psi]=[\mathrm{A}][\Psi]
$$


where

$$
[\Psi] \equiv\left[\begin{array}{c}
{\left[\psi_{1}\right]} \\
\vdots \\
{\left[\psi_{\mathrm{G}}\right]} \\
{\left[\mathrm{C}_{\mathrm{i}}\right]} \\
\vdots \\
{\left[\mathrm{C}_{\mathrm{I}}\right]}
\end{array}\right]
$$

and

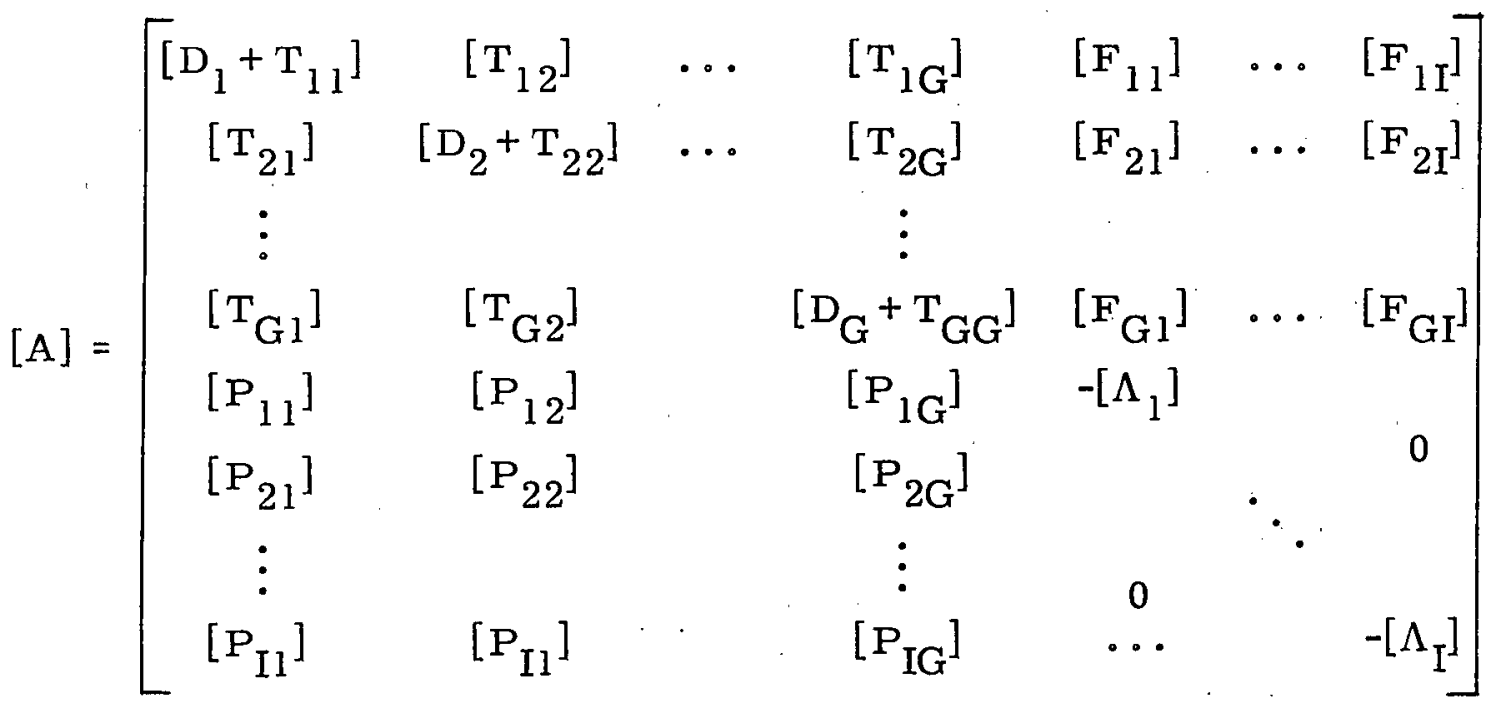

If one assumes that all the terms of the matrix [A] are constant over a time step, $\Delta t$, then the solution of the matrix equation (1.9) is given by

$$
[\Psi(\Delta t)]=\exp ([\mathrm{A}] \Delta \mathrm{t})[\Psi(0)] .
$$

Two of the three categories of direct, finite-differenced techniques involve the solution of the semi-discrete equations (1.7) and (1.8); and, consequently, are approximations to (1.10). The GAKIN METHOD (MATRIX DECOMPOSITION METHOD) ${ }^{4}$ solves the semi-discrete 
equation (1.9) by splitting the matrix [A] into four parts: [U], the upper triangular part of $[A] ;[L]$, the lower triangular part of $[A] ;[\Delta]$, that part of the sub-matrix diagonal of $[A]$ which contains the matrices $\left[D_{G}\right]$; and $[\Gamma]$, the remaining, sub-matrix diagonal part of $[A]$ which contains the matrices $\left[\mathrm{T}_{11}\right]$ through $\left[\mathrm{T}_{\mathrm{GG}}\right]$ and $-\left[\Lambda_{1}\right]$ through $-\left[\Lambda_{\mathrm{I}}\right]$.

With this splitting, equation (1.9) may be written as

$$
\frac{\mathrm{d}}{\mathrm{dt}}[\Psi]-[\Gamma][\Psi]=[\mathrm{L}+\mathrm{U}][\Psi]+[\Delta][\Psi] .^{5}
$$

Equation (1.11) then may be formally integrated over the interval $\Delta t \equiv\left(t_{p+1}-t_{p}\right)$ with the following result:

$$
\begin{aligned}
{\left[\Psi\left(t_{p+1}\right)\right]=} & \exp ([\Gamma] \Delta t)\left[\Psi\left(t_{p}\right)\right]+\int_{0}^{\Delta t} d t^{\prime} \exp \left(\left(\Delta t-t^{\prime}\right)[\Gamma]\right)[L+U]\left[\Psi\left(t_{p}+t^{\prime}\right)\right] \\
& +\int_{0}^{\Delta t} d t^{\prime} \exp \left(\left(\Delta t-t^{\prime}\right)[\Gamma]\right)[\Delta]\left[\Psi\left(t_{p}+t^{\prime}\right)\right]
\end{aligned}
$$

The GAKIN METHOD then assumes that in the first integral of equation (1.12)

$$
\left[\Psi\left(t_{p}+t^{\prime}\right)\right]=\exp \left([\omega] t^{\prime}\right)\left[\Psi\left(t_{p}\right)\right]
$$

where the terms of $[\omega]$ are typically found by utilizing one's knowledge of the change in $[\Psi]$ over the preceding time step. In the second integral, it is assumed that

$$
\left[\Psi\left(t_{p}+t^{\prime}\right)\right]=\exp \left(-[\omega]\left(\Delta t-t^{\prime}\right)\right)\left[\Psi\left(t_{p+1}\right)\right]
$$

Applying these assumptions to the integral equation (1.12) yields 


$$
\begin{aligned}
\{[I] & \left.-[\omega-\Gamma]^{-1}([I]-\exp ([\Gamma-\omega] \Delta t))[\Delta]\right\}\left[\Psi\left(t_{p+l}\right)\right] \\
& =\left\{\exp ([\Gamma] \Delta t)+[\omega-I]^{-1}(\exp ([\omega] \Delta t)-\exp ([\Gamma] \Delta t))[L+U]\right\}\left[\Psi\left(t_{p}\right)\right] .
\end{aligned}
$$

It is essentially equation (1.15) that the GAKIN METHOD tackles as an approximation to the semi-discrete equation (1.9).

Another class of approximations to equation (1.10) involves that category of direct, finite-differenced techniques known as AL TERNATINGDIRECTION SEMI-TMPLICIT TECHNIQUES. These techniques replace the time derivative in equation (1.9) by two successive forward differences over a time step, $\Delta t$ (where $\Delta t \equiv 2 h$ ). Typically, a change of variables is introduced to reduce the truncation error difficulties which plague alternating-direction splitting methods. This change of variables is in fact an exponential transformation of the form $[\Psi(t)]=\exp ([\Omega] t)[\Phi(t)]$, where $[\Omega]$ is a diagonal matrix whose terms are again chosen by utilizing one's knowledge of the past behavior of $[\psi]$. If, for each half of the time step, the matrix [A] is split into two parts and the exponentials which result from the exponential transformation are evaluated at the midpoint of the step, a matrix equation may be developed which represents a general, two-step, alternating-åirection, semi-implicit method. ${ }^{6}$ With [A] split arbitrarily into $\left[\mathrm{A}_{1}\right]+\left[\mathrm{A}_{2}\right]$ for the first half of the time step, and into $\left[\mathrm{A}_{3}\right]+\left[\mathrm{A}_{4}\right]$ for the second half, this matrix equation may be written as

$$
\left[\Psi\left(t_{p+1}\right)\right]=[B(\Omega, h)]\left[\Psi\left(t_{p}\right)\right]
$$


In the preceding equation:

$$
\begin{aligned}
{[\mathrm{B}(\Omega, \mathrm{h})]=} & \exp ([\Omega] \mathrm{h})\left\{[\mathrm{I}]-\mathrm{h}\left(\left[\mathrm{A}_{4}\right]-a[\Omega]\right)\right\}^{-1}\left\{[\mathrm{I}]+\mathrm{h}\left(\left[\mathrm{A}_{3}-\gamma[\Omega]\right)\right\}\right. \\
& \cdot\left\{[\mathrm{I}]-\mathrm{h}\left(\left[\mathrm{A}_{2}-a[\Omega]\right)\right\}^{-1}\left\{[\mathrm{I}]+\mathrm{h}\left(\left[\mathrm{A}_{1}\right]-\gamma[\Omega]\right)\right\} \exp ([\Omega] \mathrm{h})\right.
\end{aligned}
$$

where $a+\gamma=1.0$.

The specific alternating-direction, semi-implicit technique used depends on the choices one makes for $\mathrm{A}_{1}$ through $\mathrm{A}_{4}$, along with $a$ and $\gamma$. To see more clearly the various choices one may make, the matrix [A] is again split into four parts: [U], the upper triangular part of $[A] ;[L]$, the lower triangular part of $[A] ;[T]$, that part of the submatrix diagonal of matrix $[\mathrm{A}]$ which contains the matrices $\left[\mathrm{T}_{11}\right]$ through $\left[\mathrm{T}_{\mathrm{GG}}\right]$; and $[\mathrm{D}]$, the remaining sub-matrix diagonal part of $[\mathrm{A}]$ which contains the matrices $\left[D_{1}\right]$ through $\left[D_{G}\right]$ and $-\left[\Lambda_{1}\right]$ through $-\left[\Lambda_{G}\right]$.

For the SYMMETRIC, ALTERNA TING-DIRECTION IMPLICIT METHOD (SADI) the following choices are made:

$$
\begin{aligned}
& a=\gamma=0.5 \\
& {\left[\mathrm{~A}_{1}\right]=\frac{1}{2}[\mathrm{~T}]+[\mathrm{U}]+\left[\mathrm{D}_{1}\right]=\left[\mathrm{A}_{4}\right]} \\
& {\left[\mathrm{A}_{2}\right]=\frac{1}{2}[\mathrm{~T}]+[\mathrm{L}]+\left[\mathrm{D}_{2}\right]=\left[\mathrm{A}_{3}\right]}
\end{aligned}
$$

where $\left[D_{1}\right]$ contains those terms of $[D]$ associated with diffusion in one direction and one half of each term in the submatrices $\left[\Lambda_{1}\right]$ through $\left[\Lambda_{\mathrm{I}}\right] . \quad\left[\mathrm{D}_{2}\right]$ is then defined by $[\mathrm{D}]=\left[\mathrm{D}_{1}\right]+\left[\mathrm{D}_{2}\right]$.

Using these same values of $\left[D_{1}\right]$ and $\left[D_{2}\right]$, the splitting choices of the NONSYMMETRIC, ALTERNA TING DIRECTION IMPLICIT (NSADI) METHOD are 


$$
\begin{aligned}
& {\left[\mathrm{A}_{1}\right]=[\mathrm{U}]+\left[\mathrm{D}_{1}\right]} \\
& {\left[\mathrm{A}_{2}\right]=[\mathrm{T}]+[\mathrm{L}]+\left[\mathrm{D}_{2}\right]} \\
& {\left[\mathrm{A}_{3}\right]=[\mathrm{U}]+\left[\mathrm{D}_{2}\right]} \\
& {\left[\mathrm{A}_{4}\right]=[\mathrm{T}]+[\mathrm{L}]+\left[\mathrm{D}_{1}\right]}
\end{aligned}
$$

with $a=1.0$ and $\gamma=0$.

The SYMMETRIC, ALTERNATING DIRECTION EXPLICIT METHOD (SADE) involves the following choices:

$$
\begin{aligned}
& a=y=0.5 \\
& {\left[\mathrm{~A}_{1}\right]=\frac{1}{2}[\mathrm{~T}]+[\mathrm{U}]+\left[\mathrm{D}_{1}\right]=\left[\mathrm{A}_{4}\right]} \\
& {\left[\mathrm{A}_{2}\right]=\frac{1}{2}[\mathrm{~T}]+[\mathrm{L}]+\left[\mathrm{D}_{2}\right]=\left[\mathrm{A}_{3}\right]}
\end{aligned}
$$

where $\left[D_{1}\right]$ contains those stripes of $[D]$ which lie above the diagonal plus one half of each term on the diagonal and where $\left[D_{2}\right]$ contains the remaining terms of $[D]$.

The NONSYMMETRIC, ALTERNATING DIRECTION EXPLICIT METHOD (NSADE) is obtained by letting

$$
\begin{aligned}
& a=1.0 \quad \gamma=0 \\
& {\left[\mathrm{~A}_{1}\right]=[\mathrm{U}]+\left[\mathrm{D}_{1}\right]} \\
& {\left[\mathrm{A}_{2}\right]=[\mathrm{T}]+[\mathrm{L}]+\left[\mathrm{D}_{2}\right]} \\
& {\left[\mathrm{A}_{3}\right]=[\mathrm{U}]+\left[\mathrm{D}_{2}\right]} \\
& {\left[\mathrm{A}_{4}\right]=[\mathrm{T}]+[\mathrm{L}]+\left[\mathrm{D}_{1}\right]}
\end{aligned}
$$


where $\left[D_{1}\right]$ and $\left[D_{2}\right]$ are the same as for the SADE method.

All of these methods have been investigated by Donald Ferguson ${ }^{7}$ in two dimensions, and the NSADE method, which was found to be the best overall strategy, has been extended successfully to three dimensions.

The third category of direct, finite-differenced techniques to be considered here stems not from a straightforward attack on equation (1.9) but rather from a "point-kinetics" approach to equations (1.5) and (1.6). This approach, known as the $\theta-M E T H O D$, is based in part on the fact that equations (1.5) and (1.6) may be formally cast into the following "point-kinetics" form ${ }^{8,9}$ :

$$
\begin{aligned}
& \frac{\mathrm{dT}}{\mathrm{dt}}=\frac{\rho-\beta}{\Lambda} \mathrm{T}+\sum_{i=1}^{\mathrm{I}} \lambda_{i} \mathrm{C}_{\mathrm{i}} \\
& \frac{\mathrm{dC}}{\mathrm{dt}}=\frac{\beta_{\mathrm{i}}}{\Lambda} \mathrm{T}-\lambda_{i} \mathrm{C}_{\mathrm{i}} \quad \mathrm{i}=1,2, \ldots \mathrm{I} .
\end{aligned}
$$

This reduction is carried out by multiplying the terms of equations (1.5) and (1.6) by an arbitrary weighting function and integrating over space and energy. In this approach, the flux vector $[\Phi(\vec{r}, t)]$ is expressed as the product of a shape function and a scalar amplitude function (i. e., $[\Phi(\vec{r}, t)] \equiv[S(\vec{r}, t)] T(t)$ ), and the resulting values of $\Lambda(t)$, $\beta_{i}(t)$ and $\rho(t)$ are typically assumed to take on constant, average values during the time step $\Delta t$.

In the $\theta-M E T H O D$, the values of $\left[\Phi_{p+1}\right]$ are found in terms of $\left[\Phi_{p}\right]$ by casting equation (1.15) into the following, differenced ${ }^{10}$ form: 


$$
\begin{aligned}
& \frac{T_{p+l}-T_{p}}{\Delta t_{p}}=\frac{\rho_{p}-\beta}{\Lambda}\left\{{ }_{\theta}^{p}{ }_{O O} T_{p+1}+\left(1-\theta_{O O}^{p}\right) T_{p}\right\} \\
& +\sum_{i} \lambda_{i}\left\{\theta_{0 i}^{p} \mathrm{C}_{i, p+1}+\left(1-\theta_{o i}^{\mathrm{p}}\right) \mathrm{c}_{i, p}\right\} \\
& \frac{\mathrm{C}_{i, p+1}-\mathrm{C}_{\mathrm{i}, \mathrm{p}}}{\Delta \mathrm{t}_{\mathrm{p}}}=\frac{\beta_{i}}{\Lambda}\left\{\theta_{i o}^{\mathrm{p}} \mathrm{T}_{\mathrm{p}+1}+\left(1-\theta_{i o}^{\mathrm{p}}\right) \mathrm{T}_{\mathrm{p}}\right\} \\
& -\lambda_{i}\left\{\theta_{i i}^{n} C_{i, p+1}+\left(1-\theta_{i i}^{n}\right) C_{i, p}\right\} \quad i=1,2, \ldots I
\end{aligned}
$$

The $\theta^{\prime} s$ appearing in equations $(1.16)$ are parameters which are selected at each time step to improve the accuracy of the approximation. The idea behind their use may be presented by considering the following, somewhat trivial initial value problem which involves only one independent variable. The problem begins by supposing that a finite difference solution is required for

$$
\frac{d \phi(t)}{d t}=R_{p} \phi(t)
$$

where $\phi(t)$ is a scalar and $R_{p}$ is a constant.

Obviously, for this initial value problem, one may express the solution $\phi\left(t_{p+1}\right)$ at time $t_{p+1}$ exactly as

$$
\phi\left(t_{p+1}\right)=\exp \left(R_{p} \Delta t_{p}\right) \phi\left(t_{p}\right)
$$

where $\Delta t_{p} \equiv t_{p+1}-t_{p}$. Alternatively, however, one may find $\phi\left(t_{p+1}\right)$ by using a difference technique which employs a weighting parameter $\theta$. In this case, the problem becomes 


$$
\frac{\phi\left(t_{p+1}\right)-\phi\left(t_{p}\right)}{\Delta t_{p}}=R_{p}\left\{\theta^{\left.p_{\phi}\left(t_{p+1}\right)+\left(1-\theta^{p}\right) \phi\left(t_{p}\right)\right\}}\right.
$$

so that

$$
\phi\left(t_{p+1}\right)=\frac{1+R_{p} \Delta t_{p}\left(1-\theta^{p}\right)}{1-R_{p} \Delta t_{p} \theta^{p}} \phi\left(t_{p}\right)
$$

By comparing the differenced solution to the exact, it becomes clear that the difference technique will produce the exact result if

$$
{ }_{\theta}^{p}=\frac{1}{R_{p} \Delta t_{p}}+\frac{1}{1-\exp \left(R_{p} \Delta t_{p}\right)} .
$$

Clearly, then, for this simple problem a "proper" selection of the $\theta$ parameters can enable one to reduce the error of the finite difference approach.

Of course, in more complex problems the exact solution is not known a priori. Fortunately, however, the use of the $\theta^{\prime} s$ often yields fairly accurate results even if they are only approximated. And, in fact, if one makes "good estimates" of approximate $\theta^{\prime} s$ for equations (1.16), it is quite possible to significantly reduce the error associated with finite differencing equations (1.15).

At this point it is noted that in order to obtain equations (1.i6) from equations (1.5) and (1.6) one must " $\theta$-difference" $(1.5)$ and (1.6) as $^{11}$ 


$$
\begin{aligned}
& \left\{\vec{\nabla} \cdot\left[D_{p}\right] \vec{\nabla}\left[\Phi_{p+1}\right]-[A]\left[\Phi_{p+1}\right]+\sum_{j}\left(1-\beta^{j}\right)\left[x_{p}^{j}\right]\left[v_{\Sigma_{f p}^{j}}^{j}\right]^{T}\left[\Phi_{p+1}\right]\right\} \theta_{o o}^{p} \\
& +\left\{\vec{\nabla} \cdot\left[D_{p}\right] \vec{\nabla}\left[\Phi_{p}\right]-[A]\left[\Phi_{p}\right]+\sum_{j}\left(1-\beta^{j}\right)\left[x_{p}\right]\left[v_{f_{p}^{j}}^{j}\right]^{T}\left[\Phi_{p}\right]\right\}\left(1-\theta_{o o}^{p}\right) \\
& \quad+\sum_{i=1}^{I} \lambda_{i}\left\{C_{i, p+1} \theta_{o i}^{p}+C_{i, p}\left(1-\theta_{o i}^{p}\right)\right\}\left[x_{i}\right]=\frac{1}{\Delta t_{p}}[v]^{-1}\left\{\left[\Phi_{p+1}\right]-\left[\Phi_{p}\right]\right\}
\end{aligned}
$$

and

$$
\begin{gathered}
\sum_{j} \beta_{i}^{j}\left[v^{j}{ }_{f p}^{j}\right]^{T}\left\{\left[\Phi_{p+1}\right] \theta_{i o}^{p}+\left[\Phi_{p}\right]\left(1-\theta_{i o}^{p}\right)\right\}-\lambda_{i}\left\{C_{i, p+1} \theta_{i i}^{p}+C_{i, p}\left(1-\theta_{i i}^{p}\right)\right\} \\
\quad=\frac{1}{\Delta t}\left\{C_{i, p+1}-C_{i, p}\right\} \quad i=1,2, \ldots I .
\end{gathered}
$$

If one solves equation $(1.18)$ for $\mathrm{C}_{\mathrm{i}, \mathrm{p}+1}$ and eliminates this term from (1.17), the resulting $\theta$-equation takes the same form as one describing a subcritical assembly with an extended source. This equation is used in the $\theta-M E T H O D$ in the following way: Given that the values of $\left[\phi_{p}\right]$ and $C_{i, p}$ are known, the terms $P_{p}, \Lambda_{p}$, and $\beta_{i p}$ which appear in equation (1.16) may be computed. A value of $\Delta t_{p}$ is then selected and the "point-kinetics" terms previously derived are used to select the $\theta^{\prime} \mathrm{s}$ which appear in the $\theta$-equation mentioned above. This equation is then used to solve for $\left[\Phi_{p+1}\right]$ which is, in turn, used in equation (1.18) to find the $\mathrm{C}_{i, \mathrm{p}+1}$. In this manner one may step out in time, repeating the procedure for each time step.

The preceding discussion of finite-difference approaches to solving 
equations (1.5) and (1.6) is not meant to be exhaustive. Rather, the attempt here has been to categorize broadly a few of the direct approaches to solving $(1.5)$ and $(1.6)$. When this is done, it becomes apparent that the direct approaches themselves involve at least two classes of methods - those which attempt to solve the semi-discrete diffusion equation in the form of equation (1.9) and those which go about solving the finite- . differenced diffusion equations using the fact that they may be recast into a "point-kinetics" form.

Although the use of a direct methodology enables one to attach error bounds to the solution of the discretized equations over a time step, it may necessitate purchasing a considerable amount of computer time. Even moderately sized problems handling a few energy groups and a few thousand mesh points are quite expensive when more than one dimension is analyzed.

To circumvent this difficulty, modal and space-time synthesis methods have been developed to reduce the number of independent variables that must be computed and thereby reduce the length and consequently the cost of the computation.

Modal methods basically begin with the assumption that the multigroup flux vector $[\Phi(\vec{r}, t)]$ can be adequately represented during a transient as a sum of predetermined fixed shapes multiplied by coefficients which are time-dependent. Thus, for most modal expansions, the flux vector takes the form

$$
[\Phi(\vec{r}, t)] \approx \sum_{k=1}^{K}\left[\psi_{k}(\vec{r})\right]\left[T_{k}(t)\right] .
$$


If the modal method being considered "fully collapses" the energy groups, $\left[\psi_{k}(\vec{r})\right]$ is a column vector and $\left[T_{k}(t)\right]$ becomes a scalar. Alternatively, a "non-collapsed" method treats $\left[\psi_{k}(\vec{r})\right]$ as a diagonal matrix and $\left[\mathrm{T}_{\mathrm{k}}(\mathrm{t})\right]$ as a column vector. "Fully collapsing" implies that the group-to-group flux ratios are no longer completely free to vary independently. "Non collapsing," on the other hand, purchases this freedom in part by increasing the number of time-dependent equations to be solved.

In general, the kind of approximation defined by equation (1.19) is not valid at all $\vec{r}$ and for all $t$. By the very nature of the approximation, a real, linear vector space is generated by one's choice of the trial function $\left[\psi_{k}(\vec{r})\right]$. And, the solution of the approximate equations which result from the application of equation (1.19) must come from this vector space. Unfortunately, the space cannot usually be expected to contain the exact solution to the time-dependent, multigroup diffusion equation. To circumvent this difficulty, one may apply the method of weighted residuals (or, alternatively, variational techniques) in order to select from the approximate solution space that solution which lies "closest" to the exact solution.

The application of the method of weighted residuals is accomplished by substituting the modal approximation, equation (1.19), into the timedependent, multigroup diffusion equations. The terms of the resulting set of equations are then premultiplied by a series of weighting functions and integrated over all space. The weighting matrices, $\left[\mathrm{w}_{\mathrm{p}}(\overrightarrow{\mathrm{r}})\right]$ must, of course, be of a nature and number so as to generate the exact number of equations necessary to determine the unknown coefficients in 
the right-hand side of equation (1.19).

The type of modal method employed is determined primarily by the selection of the trial functions $\psi_{k}(\vec{r})$.

The $\psi_{\mathrm{k}}(\overrightarrow{\mathrm{r}})$ which âre:defined by

$$
\nabla^{2} \psi_{\mathrm{k}}(\overrightarrow{\mathrm{r}})+\mathrm{B}_{\mathrm{k}}^{2} \psi_{\mathrm{k}}(\overrightarrow{\mathrm{r}})=0
$$

are known as HELMHOLTZ MODES. ${ }^{12}$ They have the advantage of being complete, orthogonal functions which are easily tabulated. Unfortunately, however, a very large number of modes must generally be used to describe the reactor adequately.

Before investigating other choices of trial functions, a recasting of equations $(1.5)$ and $(1.6)$ is in order. To accomplish this, the following matrices are defined:

$$
\begin{aligned}
& {[L] \equiv-\{\vec{\nabla} \cdot[D(\vec{r}, t)] \vec{\nabla}-[A(\vec{r}, t)]\}} \\
& {[M] \equiv \sum_{j}\left\{\left(1-\beta^{j}\right)\left[x_{\rho}^{j}\right]+\sum_{j} \beta_{i}^{j}\left[x_{i}\right]\right\}\left[v^{j_{\Sigma}} j_{f}^{j}\right] T} \\
& {\left[M_{i}^{d}\right] \equiv \sum_{j} \beta_{i}^{j}\left[x_{i}\right]\left[v^{j_{\Sigma}}{ }_{f}^{j}(\vec{r}, t)\right]^{T}} \\
& {\left[M^{d}\right] \equiv \sum_{i}\left[M_{i}^{d}\right]}
\end{aligned}
$$

Applying the definitions to equations (1.5) and (1.6) yields

$$
\left[-L+M-M^{d}\right][\Phi]+\sum_{i=1}^{I}\left[x_{i}\right] \lambda_{i} C_{i}=\frac{\partial}{\partial t}[v]^{-1}[\Phi]
$$


and

$$
\left[M_{i}^{d}\right][\Phi]-\left[x_{i}\right] \lambda_{i} C_{i}=\left[x_{i}\right] \frac{\partial C_{i}}{\partial t} \quad i=1,2, \ldots I
$$

If one denotes steady-state conditions by a "o" subscript, then LAMBDA MODES ${ }^{13}$ are defined by

$$
\left[L_{0}\right]\left[\Phi_{\lambda n}\right]=\frac{1}{\lambda_{n}}\left[M_{0}\right]\left[\Phi_{n}\right]
$$

and OMEGA MODES ${ }^{14}$ are defined by

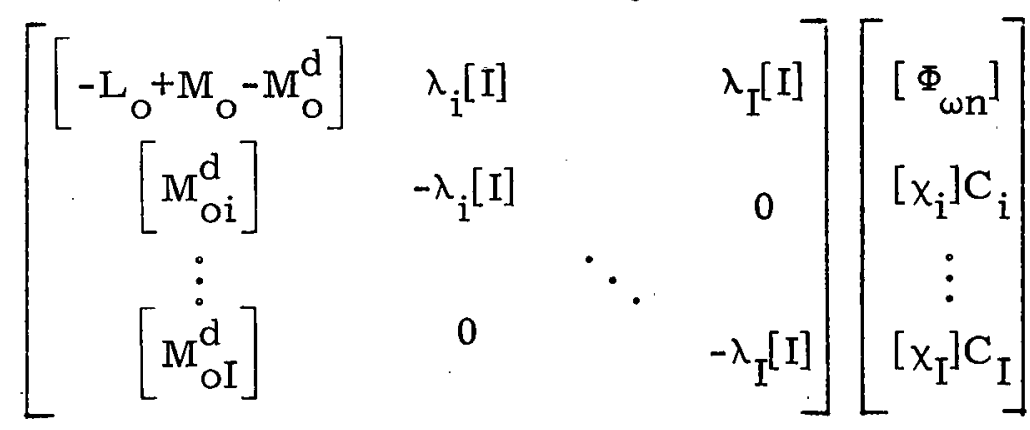

$$
\begin{aligned}
& =\omega_{n}\left[\begin{array}{cccc}
{\left[v_{0}\right]^{-1}} & & & \\
& {[I]} & 0 & \\
0 & & \ddots & \\
& & & {[I]}
\end{array}\right]\left[\begin{array}{c}
{\left[\Phi_{\omega n}\right]} \\
{\left[x_{i}\right] C_{i}} \\
\vdots \\
{\left[x_{I}\right] C_{I}}
\end{array}\right]
\end{aligned}
$$

LAMBDA and OMEGA MODES have the advantage that they can be tailored to a particular problem by using $\left[\mathrm{L}_{\mathrm{O}}\right]$ and $\left[\mathrm{M}_{\mathrm{O}}\right]$ appropriate for that problem. Consequently the number of such modes required for an adequate approximation is much smaller than the corresponding number of HELMHOLTZ MODES. Unfortunately, these modes are difficult to calculate; and, since they correspond to only one of the physical states which the reactor experiences during a transient, they will be poor 
choices if the reactor deviates substantially from that state.

All of the choices of trial functions considered so far involve a set of modes which correspond to only one operator. TIME SYNTHESIS ${ }^{15}$ is a modal approximation that uses modes which are associated with a set of operators. Typically, the elements of the $K,\left[\psi_{k}(\vec{r})\right]$ matrices are fundamental modes belonging to a set of operators chosen to represent the reactor during the course of the transient. The emphasis is then to use as much of one's knowledge and intuition about the dynamic behavior of the reactor as possible in the selection of the set of operators.

The great advantage of synthesis procedures is that the trial functions can be found by standard static methods and can be tailored very directly to the problem at hand. In many cases, therefore, few of them are required. As an important result, it becomes possible to solve an adequate approximation to the space-time diffusion equation with great detail, accurately, and at a reasonable cost.

There are, however, some important objections to the timesynthesis method. The modes do not form a complete set. There is no orthogonality relationship among the modes. The selection of the reactor conditions yielding the trial functions requires some intuitive judgment about the dynamic characteristics of the reactor. In threedimensions, the cost to find three-dimensional trial and weight functions may be substantial. A great deficiency is the lack of satisfactory error bounds. Practically, care must be exercised to avoid using linearly dependent trial functions.

The trade-off in advantages and disadvantages of the TIME 
SYNTHESIS METHOD depends on the type of problem one is attempting to solve. For modeling the slow removal or insertion of a control rod, the separability of the spatial and temporal behavior of the flux is a poor approximation. For a small, uniform change in the boron content of boron-poisoned coolant, the approximation would be too sophisticated. However, for a range of interesting problems, the approximation is quite attractive. For example, the method is well suited to describe the flux behavior following a cold water injection, a loss of flow, or the rapid ejection or insertion of a control rod.

The second category of indirect techniques for tackling equations $(1.5)$ and $(1.6)$ springs from an attempt to circumvent the difficulties imposed by time synthesis of finding three-dimensional trial and weight functions for the entire reactor and of knowing a priori something about the three-dimensional temperature profile of the reactor during the transient. Procedures belonging to this category are usually referred to as SPACE-TIME SYNTHESIS methods.

The idea behind space-time synthesis methods is to extend the notion of time-synthesis so that one may select trial functions which apply only to a region of the reactor. A consequence of doing this is that the coefficients of expansion become functions of position as well as time.

There are many types of space-time synthesis. In NODAL ANALYSIS, ${ }^{16}$ one partitions the reactor into several subregions, $R_{n}$. For the reactor, then, the flux is approximated by

$$
\left[\Phi(\vec{r}, t]=\sum_{n=1}^{N} \Gamma_{n}(\vec{r})[\psi(\vec{r})]\left[T^{(n)}(t)\right]\right.
$$


where

$$
\Gamma_{n}(\vec{r})= \begin{cases}1 & n \subset R_{n} \\ 0 & n \not R_{n}\end{cases}
$$

SINGLE-CHANNEL SYNTHESIS ${ }^{17}$ may use either continuous or discontinuous trial functions. In either case, the motivation is to make use of the axially homogeneous nature of many reactors. The idea is to extend the synthesis methodology by representing $[\Phi(\vec{r}, t)]$ as a linear combination of two-dimensional flux shapes appropriate to radial slices of the core taken at different elevations and unknown functions of height and time. For continuous trial functions, the assumption is

$$
[\Phi(\vec{r}, t)]=\sum_{k=1}^{K}\left[\psi_{k}(x, y)\right]\left[T_{k}(z, t)\right]
$$

The use of discontinuous trial functions permits one to use different sets of the expansion functions $\left[\psi_{k}(x, y)\right]$ at different elevations. Thus the flux vector is approximated as

$$
[\Phi(\vec{r}, t)]=\sum_{k=1}^{K}\left[\psi_{k n}(x, y)\right]\left[T_{k n}(z, t)\right] \quad z_{n} \leqslant z \leqslant z_{n+1} \quad n=1,2, \ldots N
$$

MULTICHANNEL SYNTHESIS ${ }^{18}$ is an extension of the idea of singlechannel synthesis whereby the $x-y$ plane is itself partitioned into $M$ regions $R_{m}$. In the discontinuous trial function expansion given above $\left[\psi_{\mathrm{k}, \mathrm{n}, \mathrm{m}}(\mathrm{x}, \mathrm{y})\right]$ is defined to be $\left[\psi_{\mathrm{k}, \mathrm{n}}(\mathrm{x}, \mathrm{y})\right]$ in $\mathrm{R}_{\mathrm{m}}$ and zero elsewhere. Thus the expansion becomes

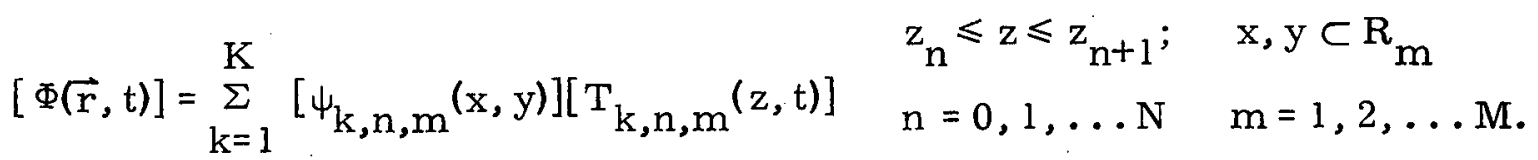


If a decision is made to use an indirect approach to solving equations $(1.5)$ and $(1.6)$, another decision must then be made as to how one should go about solving the resulting, approximate equations. Recalling the structure that was observed with the development of direct approaches, one can see that two choices are open. One may apply an indirect approach in a straightforward way to equations (1.5) and (1.6) and solve the resulting equations, or one may cast such equations into a "point kinetics" form and go about the task of solving these reformulated equations. In this latter approach, the development of multimode kinetics equations of a point kinetics form has been analyzed by Fuller, Hetrick and Meneley ${ }^{19}$ and shown to be the result of the application of a weighted residual methodology to the spatial domain. If the reactor is divided into $M$ channels, and for each channel it is assumed that

$$
\left[\Phi_{m}(\vec{r}, t)\right]=\sum_{k=1}^{K}\left[\psi_{m, k}(\vec{r}, t)\right]\left[T_{m, k}(t)\right]
$$

then the following equations may be derived by applying this expansion to the time-dependent, multigroup, multiregion diffusion equations (along with boundary and interface conditions), premultiplying the resulting terms by an appropriate set of weighting functions, and performing spatial integrations over each channel:

$$
\frac{d}{d t}\left[\begin{array}{c}
{[T(t)]} \\
{\left[C_{1}(t)\right]} \\
\vdots \\
{\left[C_{I}(t)\right]}
\end{array}\right]=\left[\begin{array}{cccc}
{[\Lambda]^{-1}[\rho-\beta]} & \lambda_{1}[I] & \cdots & \lambda_{I}[I] \\
{[\Lambda]^{-1}\left[\beta_{1}\right]} & -\lambda_{1}[I] & & 0 \\
\vdots & 0 & \ddots & \\
{[\Lambda]^{-1}\left[\beta_{I}\right]} & & & -\lambda_{I}[I]
\end{array}\right]\left[\begin{array}{c}
{[T(t)]} \\
{\left[C_{1}(t)\right]} \\
\vdots \\
{\left[C_{I}(t)\right]}
\end{array}\right]
$$


where the size of the sub-matrices depends on the kind of group collapsing scheme chosen for the expansion (1.22).

The selection of the kind of trial functions which appear in (1.22) determines the type of indirect method to be employed. If the entire reactor is treated as one channel containing a single time-independent shape function, then the sub-matrices of (1.23) become scalars and the equation becomes merely the familiar point-kinetics equation. If only one channel is considered and a single shape function is defined by any time step, p, by

$$
[\mathrm{L}][\Phi(\overrightarrow{\mathrm{r}}, \mathrm{p})]=\frac{1}{\lambda_{\mathrm{p}}}[\mathrm{M}][\Phi(\overrightarrow{\mathrm{r}}, \mathrm{p})]
$$

(where [L] and [M] are operators for the perturbed reactor in the $\mathrm{p}^{\text {th }}$ time step), then the ADIABATIC METHOD ${ }^{20}$ is being employed. Or, if one attempts to improve on the ADIABATIC METHOD by using

$$
\begin{aligned}
& {[-L+M][\Phi(\vec{r}, p)]-\sum_{i} \frac{\omega_{i}(\vec{r}, p-1)}{\lambda_{i}+\omega_{i}(\vec{r}, p-1)}[\Phi(\vec{r}, p-1)]} \\
& \quad=[v]^{-1} \frac{1}{\Delta t_{p}}[\Phi(\vec{r}, p)-\Phi(\vec{r}, p-1)]
\end{aligned}
$$

to define the shape function, then the QUASI-STATIC METHOD ${ }^{21}$ has been adopted.

Nodal methods improve on the point reactor model by considering one spatial trial function in each channel. Thus equation (1.21) becomes

$$
\left[\Phi_{m}(\vec{r}, t)\right]=\left[\psi_{m, i}(\vec{r}, t)\right]\left[T_{m, i}(t)\right] \quad m=1,2, \ldots M
$$

Alternatively, the reactor may be treated as one channel and omega 
modes on Helmholtz modes employed. Or, finally, one may choose to adopt single-channel synthesis, multichannel synthesis, or time synthesis in developing the multimode kinetics equations of the point-kinetics form.

This section has been concerned with a review of several of the current techniques of approximating equations (1.5) and (1.6). The remainder of the thesis deals with one of these - the solution of the multimode kinetics equations of the point-kinetics form.

\subsection{A Development of the Multimode Kinetics Equations}

In this section, the multimode kinetics equations will be developed in a point-kinetics form by the application of time synthesis to equations (1.5) and $(1,6)$. There are two reasons for doing this at this time. One is to clarify the preceding discussion of using the method of weighted residuals to develop the multimode kinetics equations in the form of equations (1.23). The other is to help set the stage for the next chapter which will discuss how these equations are to be solved.

As discussed earlier, the application of time synthesis is made by approximating the flux vector with the expansion given by equation (1.19). This approximation is then introduced into equations (1.20) and (1.21). The resulting equations are then premultiplied by a set of arbitrary, time-independent weight functions $\left[\mathrm{w}_{\mathrm{p}}(\overrightarrow{\mathrm{r}})\right]$ and integrated over space. If the weighting functions are the neutron importance functions, a reduction is made in the errors arising from the approximate nature of the spatial trial functions employed.

The result is 


$$
\begin{aligned}
\sum_{k=1}^{K}\left\{\int_{v}\left[W_{p}\right]\left[-L+M-M^{d}\right]\left[\psi_{k}\right] d v\right\}\left[T_{k}\right]+\sum_{i=1}^{I} \int_{v}\left[W_{p}\right]\left[x_{i}\right] \lambda_{i} C_{i} d v \\
\quad=\sum_{k=1}^{K}\left\{\int_{v}\left[W_{p}\right][v]^{-1}\left[\psi_{k}\right] d v\right\} \frac{d}{d t}\left[T_{k}(t)\right] \quad p=1,2, \ldots K
\end{aligned}
$$

and

$$
\begin{aligned}
& \sum_{k=1}^{K}\left\{\int_{v}\left[w_{p}\right]\left[M_{i}^{d}\right]\left[\psi_{k}\right] d v\right\}\left[T_{k}\right]-\lambda_{i} \int_{v}\left[w_{p}\right]\left[x_{i}\right] C_{i} d v \\
& \quad=\frac{1}{d t} \int_{v}\left[w_{p}\right]\left[x_{i}\right] C_{i} d v \quad p=1,2, \ldots K ; \quad i=1,2, \ldots I
\end{aligned}
$$

If equation (1.19) is applied in a non-collapsed manner, then equations (1.24) and (1.25) represent $G \times K+G \times K \times I$ equations which can be used to find the $G \times K$ unknown $T_{g k}(t)$ 's and the $G \times K \times I$ unknown $\int d v W_{p g}(\vec{r}) x_{i g} C_{i}(\vec{r}, t)^{\prime} s$.

Equations (1.24) and (1.25) may be transformed into the pointkinetics form in several ways. This flexibility stems from the fact that in the point-kinetics formulation only ratios like $\frac{\rho}{\Lambda}$ and $\frac{\beta}{\Lambda}$ must be specified.

Here, the following definitions are made:

$$
\begin{aligned}
& {[\rho]_{\mathrm{pk}} \equiv \int_{\mathrm{v}}\left[\mathrm{W}_{\mathrm{p}}\right][-\mathrm{L}+\mathrm{M}]\left[\psi_{\mathrm{k}}\right] \mathrm{dv}} \\
& {\left[\beta_{\mathrm{i}}\right]_{\mathrm{pk}} \equiv \int_{\mathrm{v}}\left[\mathrm{w}_{\mathrm{p}}\right]\left[\mathrm{M}_{\mathrm{i}}^{\mathrm{d}}\right]\left[\psi_{\mathrm{k}}\right] \mathrm{dv}} \\
& {[\Lambda]_{\mathrm{pk}} \equiv \int_{\mathrm{v}}\left[\mathrm{W}_{\mathrm{p}}\right][\mathrm{v}]^{-1}\left[\psi_{\mathrm{k}}\right] \mathrm{dv}} \\
& \left\{[\Lambda]\left[\mathrm{C}_{\mathrm{i}}\right]\right\} \equiv \operatorname{col}:\left\{\int_{\mathrm{v}}\left[\mathrm{w}_{\mathrm{i}}\right]\left[\mathrm{x}_{\mathrm{i}}\right] \mathrm{C}_{\mathrm{i}} \mathrm{dv} \ldots \int_{\mathrm{v}}\left[\mathrm{w}_{\mathrm{k}}\right]\left[\mathrm{x}_{\mathrm{i}}\right]\left[\mathrm{C}_{\mathrm{i}}\right] \mathrm{dv}\right\}
\end{aligned}
$$


Applying these definitions to equations $(1.29)$ and (1.25) yields

$$
\begin{aligned}
& \frac{d}{d t}[T]=[\Lambda]^{-1}[\rho-\beta][T]+\sum_{i} \lambda_{i}\left[C_{i}\right] \\
& \frac{d}{d t}\left[C_{i}\right]=[\Lambda]^{-1}\left[\beta_{i}\right][T]-\lambda_{i}\left[C_{i}\right] \quad i=1,2, \ldots I
\end{aligned}
$$

or, equivalently, equation (1.23).

Noticing that equation (1.23) may be written as

$$
\frac{\mathrm{d}}{\mathrm{dt}}[\Psi]=[\mathrm{A}][\Psi]
$$

leads one to conclude that if the terms of the sub-matrices of equation (1.23) are constant over a time step, then the solution to the multimode kinetics equations in a point-kinetics form over a time step $\Delta t\left(=t_{p+1}-t_{p}\right)$ is

$$
\left[\psi_{p+1}\right]=\exp (\Delta t[\bar{A}])\left[\psi_{p}\right]
$$

The next chapter discusses how one might approach solving equation (1.26) and the kinds of approaches that one may choose to approximate equation (1.29). 
CHAPTER II

\section{A NEW APPROACH TO SOLVING THE MULTIMODE KINETICS EQUATIONS}

The preceding chapter was concerned in part with the development of the multimode kinetics equations and the casting of these equations into a point-kinetics form. It is the purpose of this chapter to present a brief review of the current techniques used in solving such equations and then to develop a new approach to their solution, based on approximating the exponential of equation (1.29).

\subsection{A Review of Techniques for Solving Multimode Kinetics}

Equations Which Have Been Cast in a Point-Kinetics

\section{Form}

The following discussion of the current methods for solving the multimode kinetics equations in the form of equation (1.29) begins with a consideration of methods of solving the space-independent, pointkinetics equations themselves. There are at least two good reasons for taking the time now to do this. First, as was pointed out in Chapter I, the point-kinetics equations in fact represent a specialization of the multimode kinetics equations produced by treating the entire reactor as one channel containing a single, time-independent mode. Second, since it has been demonstrated that the multimode kinetics equations can indeed be cast as matrix generalizations of the point-kinetics equations, there is some cause to hope that one might look to the methodology of solving the space-independent equations in order to obtain a few clues as to how to go about solving the multimode kinetics 
equations. That this is the case will be demonstrated as the chapter unfolds。

Because of the difficulty of analytic approaches 22,23 a considerable number of approximate methods have been devised over the years to solve the space-independent, point-kinetics equations. Most of these methods fall into the following six categories:

(1) methods based essentially on Taylor series expansions ${ }^{24,25}$

(2) methods based on convolution integrals using numerical integration 26,27

(3) methods based on integral equation formulations and approximation of the integrand $28-31$

(4) methods based on some approximation of matrix exponentials $32-34$

(5) methods based on extrapolation of low-order approxima tions 35

(6) methods based on finite differencing 36,37

Of these methods, only one appears to have been extended to cases where the parameters of the point-kinetics equations are themselves square matrices. Generalizing the earlier work of Brittan ${ }^{38}$ and Kaganove, ${ }^{28}$ Fuller, Meneley, and Hetrick ${ }^{39}$ have successfully employed the method of undetermined parameters in the temporal integration of the multimode kinetics equations. This approach stems from the fact that the matrix equations (1.26) and (1.27) may be reformed into a single, integral matrix equation by performing the following operations. First, it is noted that the I equations (1.27) may be substituted into $(1.26)$ to eliminate the $\sum_{i=1}^{I} \lambda_{i}\left[C_{i}(t)\right]$ term and thereby yield: 


$$
\frac{d}{d t}[T(t)]=[\Lambda]^{-1}[\rho][T(t)]-\sum_{i=1}^{I} \frac{d}{d t}\left[C_{i}(t)\right]
$$

Secondly, in order to deal with the $\frac{d}{d t}\left[C_{i}(t)\right]$ terms in $(2.1)$, equation (1.27) is formally integrated over the interval $t_{p} \leqslant t \leqslant t_{p+l}$ to yield:

$$
\begin{aligned}
{\left[C_{i}(t)\right]=} & {\left[C_{i}\left(t_{p}\right)\right] \exp \left[-\lambda_{i}\left(t-t_{p}\right)\right] } \\
& +\int_{t_{p}}^{t}[\Lambda]^{-1}\left[\beta_{i}\left(t^{\prime}\right)\right]\left[T\left(t^{\prime}\right)\right] \exp \left[-\lambda_{i}\left(t-t^{\prime}\right)\right] d t^{\prime}
\end{aligned}
$$

If one then differentiates (2.2), the resulting expression,

$$
\begin{aligned}
\frac{d}{d t}\left[C_{i}(t)\right]= & -\lambda_{i}\left[C_{i}\left(t_{p}\right)\right] \exp \left[-\lambda_{i}\left(t-t_{p}\right)\right]+[\Lambda]^{-1}\left[\beta_{i}(t)\right][T(t)] \\
& -\lambda_{i} \int_{t_{p}}^{t}[\Lambda]^{-1}\left[\beta_{i}\left(t^{\prime}\right)\right]\left[T\left(t^{\prime}\right)\right] \exp \left[-\lambda_{i}\left(t-t^{\prime}\right)\right] d t^{\prime},
\end{aligned}
$$

may be substituted into equation (2.1) to obtain the following matrix, integral equation:

$$
\begin{aligned}
\frac{d}{d t}[T(t)]= & {[\Lambda]^{-1}[\rho-\beta][T(t)]+\sum_{i=1}^{I} \lambda_{i}\left\{\left[C_{i}\left(t_{p}\right)\right] \exp \left[-\lambda_{i}\left(t-t_{p}\right)\right]\right.} \\
& \left.+\int_{t_{p}}^{t}[\Lambda]^{-1}\left[\beta_{i}\left(t^{\prime}\right)\right]\left[T\left(t^{\prime}\right)\right] \exp \left[-\lambda_{i}\left(t-t^{\prime}\right)\right] d t^{\prime}\right\}
\end{aligned}
$$

The method of undetermined parameters is then applied to the solution of equation (2.4) by assuming that, in the time interval $t_{p} \leqslant t \leqslant t_{p+l}$, the matrices $[T(t)]$ may be expressed as 


$$
[T(t)]=\sum_{k=0}^{k}\left[A_{k}\right](t-t)^{k}
$$

The unknown elements of the vectors $\left[A_{k}\right]$ are then found by substituting equation (2.5) into (2.4) and applying the method of weighted residual to the result. That is to say, the terms of the resulting equation are multiplied by a set of weighting functions and integrated over the time interval.

Typically, the application of the weighting functions forces one to require that the residual vanish at $K$ points $\left(t_{1}, \ldots t_{K}\right)$, all lying within the time interval. Thus, in stepping out in time from $t_{p}$ to $t_{p+l}, K$ integrals must be evaluated. In this way $\mathrm{K}$ simultaneous, matrix equations are generated, from which the $\left[A_{k}\right]$ may be found.

The accuracy of this procedure depends both on the degree of the piecewise matrix polynomial used in equation (2.5) and on the kind of weighting functions selected fro the temporal integration. Fuller, Meneley, and Hetrick ${ }^{39}$ chose second degree polynomials in an attempt to balance consistency requirements and calculational effort. In considering the sensitivity of the result on the choice of weighting functions, Fuller ${ }^{40}$ has discussed three possibilities. Collocation weighting uses the Dirac delta functions, $\delta\left(t-t_{k}\right) ; k=1, \ldots k$. Subdomain weighting, which was used by Brittan ${ }^{38}$ and Kaganove ${ }^{28}$ for point kinetics, uses the unit step functions $u(t)-u\left(t-t_{k}\right) ; k_{1}=1, \ldots K$. (Here it is suggested that a good choice for the subdomains is $\left.t_{k}=t_{p}+\frac{\left(t_{p+1}-t_{p}\right)}{2^{k-1}} ; k=1, \ldots K.\right)$ Thirdly, Galerkin weighting uses the trial functions $\left(t-t_{p}\right)^{k} ; k=$ $1, \ldots \mathrm{K}$ as weighting functions. Of these three choices, Galerkin 
weighting appears to be the most capable of giving accurate solutions.

2.2 An Alternate Approach to the Solution of the Multimode

Kinetics Equations - The Approximation of $\exp (\Delta t[A])$

Recently a new method of solving the point-kinetics equations was developed by da Nóbrega ${ }^{34}$ which proved to be fast and accurate and which has the ability to reproduce all the features of space-independent transients, including the prompt jump. Crucial to the success of this new method was the development of an analytic technique to inver polynomials of the point-kinetics matrix. This inversion has direct applicability to the Padé approximations for the exponential; and, because of its success in solving the point-kinetics equations, it forms the basis for motivating one to consider the extension of the method to solving the multimode kinetics equations.

The remainder of this chapter deals with this extension. Specifically, it deals with the application of an analytic inversion to both the Padé $(2,0)$ and a modified Padé $(1,1)$ approximation to the exponential of equation (1.29). It is left to the rest of this section to provide some insight into these two approximations and to develop more clearly the motivation behind their selection and use.

Late in the nineteenth century Padé ${ }^{41}$ realized that any analytic function

$$
f(x)=a_{0}+a_{1} x+a_{2} x^{2}+\ldots
$$

in the neighborhood of the origin might be approximated utilizing the now classical analysis tool of rational approximation. Essentially, the 
idea is to express $f(x)$ approximately by

$$
f(x) \approx \frac{n_{p, q^{(x)}}}{d_{p, q^{(x)}}} \equiv r_{p, q^{(x)}}
$$

where $n_{p, q}(x)$ and $d_{p, q}(x)$ are, respectively, polynomials of degree $q$ and $p$ in $x$ and where it is assumed that $d_{p, q}(0) \neq 0$. One then selects for each pair of positive integers $p$ and $q$ those polynomials $n p, q(x)$ and $d_{p, q}(x)$ such that a Taylor series expansion of $r_{p, q}(x)$ agrees with as many terms of (2.6) as possible. The coefficients of the polynomials $n_{p, q}(x)$ and $d_{p, q}(x)$ are determined from the fact that

$$
\mathrm{d}_{\mathrm{p}, \mathrm{q}}(\mathrm{x}) \mathrm{f}(\mathrm{x})-\mathrm{n}_{\mathrm{p}, \mathrm{q}}(\mathrm{x})=\mathscr{O}\left(|\mathrm{x}|^{\mathrm{p}+\mathrm{q}+1}\right) \quad \text { as }|\mathrm{x}| \rightarrow 0
$$

This equation gives rise to $p+q+1$ linear equations from which the $p+q+1$ essential unknown coefficients of $r_{p, q}(x)$ may be found.

For the function $f(x) \equiv \exp (x)$, Hummel and Seebeck ${ }^{43}$ have found that a Pade $(p, q)$ approximation is composed of

$$
n_{p, q}(x)=\sum_{k=0}^{q} \frac{(p+q-k) ! q !}{(p+q) ! k !(q-k) !}(+x)^{k}
$$

and

$$
d_{p, q}(x)=\sum_{k=0}^{p} \frac{(p+q-k) ! p !}{(p+q) ! k !(p-k) !}(-x)^{k}
$$

A Padé $(2,0)$ approximation for $\exp (\mathrm{x})$ is thus given by

$$
\exp (x) \approx \frac{1}{1-x+\frac{x^{2}}{2}}
$$


$\operatorname{Varga}^{42}$ notes that the Padé rational approximations for $\exp (\mathrm{x})$ can can directly lead one to consider matrix approximations to $\exp (\Delta \mathrm{t}[\mathrm{A}])$. To see this one merely has to replace formally the $\mathrm{x}$ variable by $\Delta t[\mathrm{~A}]$ and let

$$
\exp (\Delta t[A]) \approx\left[d_{p, q}([A] \Delta t)\right]^{-1}\left[n_{p, q}([A] \Delta t] \equiv E_{p, q}(\Delta t[A])\right.
$$

Thus one is led to the Padé $(2,0)$ and Pade $(1 ; 1)$ approximations of $\exp (\Delta t[A])$. These are, respectively:

$$
\mathrm{E}_{2,0}(\Delta \mathrm{t}[\mathrm{A}]) \equiv\left[[\mathrm{I}]-\Delta \mathrm{t}[\mathrm{A}]+\frac{\Delta \mathrm{t}}{2}[\mathrm{~A}]^{2}\right]^{-1}
$$

and

$$
\mathrm{E}_{1,1}(\Delta \mathrm{t}[\mathrm{A}]) \equiv\left[[\mathrm{I}]-\frac{\Delta \mathrm{t}}{2}[\mathrm{~A}]\right]^{-1}\left[[\mathrm{I}]+\frac{\Delta \mathrm{t}}{2}[\mathrm{~A}]\right]
$$

As a final point in the development of these two matrix approximations, it is noted that over the time interval $\Delta t$, the parameters of the multimode kinetics matrix [A] may change as the physical properties of the reactor modeled by $(1.28)$ change. If one denotes as $\left[A\left(t_{p}\right)\right]$ the matrix associated with the reactor at the beginning of the time step when $t=t_{p}$ and refers to the matrix $\Delta t$ seconds later as $\left[A\left(t_{p}+\Delta t\right)\right]$, then the requirement imposed when the solution of $(1.28)$ is expressed as (1.29) (i. e., the requirement that [A] be constant over a time step) may be maintained by assuming that in equation (1.29) [ $\bar{A}]$ over $\Delta t$ is equal to ${ }_{2}^{1}\left[\left[A\left(t_{p}\right)\right]+\left[A\left(t_{p}+\Delta t\right)\right]\right]$. In the present work, this assumption is made in the case of the $\mathrm{E}_{2,0}(\Delta \mathrm{t}[\mathrm{A}])$ approximation.

In the case of the $\mathrm{E}_{1,1}(\Delta \mathrm{t}[\mathrm{A}])$ approximation, however, an alternative approach is possible. To account for the change in [A] over $\Delta t$, 
one may modify the $E_{1,1}(\Delta t[A])$ approximation in the following way:

$$
E_{1,1}^{\prime}(\Delta t[A]) \equiv\left[[I]-\frac{\Delta t}{2}\left[A\left(t_{p}+\Delta t\right]\right]^{-1}\left[I+\frac{\Delta t}{2}\left[A\left(t_{p}\right)\right]\right]\right.
$$

Here, $E_{1,1}^{\prime}(\Delta t[A])$ clearly becomes $E_{1,1}(\Delta t[A])$ for constant $[A]$.

Equations (2.9) and (2.11) form the basis in the present work for approximating the exponential of equation (1.29). The motivation behind their selection will unfold in a logical manner as the following discussion of the properties of $E_{2,0}(\Delta t[A])$ and $E_{1,1}^{\prime}(\Delta t[A])$ develops.

The discussion begins with a mathematically precise statement of the problem at hand (see Richtmyer and Morton ${ }^{45}$ ). For the purposes of the present work, it is required that one find a one-parameter family $[\psi(t)]$ of elements of the Banach space $\mathscr{B}$ such that the equation

$$
\frac{d}{d t}[\psi(t)]=[A(t)][\psi(t)] \quad 0 \leqslant t \leqslant T
$$

is satisfied. In this initial value problem, $t$ is a real parameter, $[A(t)]$ is a linear operator whose domain is restricted to the set of all real numbers, and $[\psi(0)] \equiv \psi_{0}$, where $\left[\psi_{0}\right]$ is a given element of the $\mathscr{B}$ space which may be used to describe the initial spatial distribution of the neutron population of the reactor modeled by $(1.28)$.

A genuine solution to equation $(1.28)$ is the family of $[\psi(t)]$ which lie in the domain of $[A(t)]$ for each $t$ in the interval $0 \leqslant t \leqslant T$ and which satisfy

$$
\left\|\frac{[\psi(t+\Delta t)]-[\psi(t)]}{\Delta t}-[A(t)][\psi(t)]\right\| \rightarrow 0 \quad \text { as } \Delta t \rightarrow 0 \quad 0 \leqslant t \leqslant T
$$


The following approximation for $(1.28)$ is now constricted:

$$
\left[\psi\left(t_{p+1}\right)\right]=\left[E(\Delta t]\left[\psi\left(t_{p}\right)\right]\right.
$$

where, in the present work, $[\mathrm{E}(\Delta \mathrm{t})]$ may be either $\mathrm{E}_{2,0}(\Delta \mathrm{t}[\mathrm{A}])$ or $E_{1,1}^{\prime}(\Delta t[A])$ and $\Delta t \equiv t_{p+1}-t_{p} \cdot$ Clearly, as $\Delta t \rightarrow 0$, one would like the expression $\frac{\left[\psi\left(t_{p+1}\right)\right]-\left[\psi\left(t_{p}\right)\right]}{\Delta t}$ to be an approximation to the time derivative $\frac{d}{d t}[\psi(t)]$. The implication of this requirment is that, as $\Delta t \rightarrow 0$, the ratio

$$
\frac{[E(\Delta t)][\psi(t)]-[\psi(t)]}{\Delta t}
$$

be an approximation, in some sense, to $[A(t)][\psi(t)]$. What is being considered here in rather loose terms is the topic of consistency (see Lax and Richtmyer ${ }^{44}$ ). More formally, one may define this concept in the following way ${ }^{45}$ :

DEFINITION 2.1. The family $[\mathrm{E}(\Delta \mathrm{t})]$ of operators provides a consistent approximation for the initial value problem $\frac{d}{d t}[\psi(t)]=[A(t)][\psi(t)]$ if, for every $[\psi(t)]$ in some class $\phi$ of genuine solutions whose initial elements $[\psi(0)]$ are dense in the $\mathscr{B}$ space,

$$
\left\|\left\{\frac{[\mathrm{E}(\Delta \mathrm{t})]-[\mathrm{I}]}{\Delta \mathrm{t}}-[\mathrm{A}(\mathrm{t})]\right\}[\psi(\mathrm{t})]\right\| \rightarrow 0 \quad \text { as } \Delta t \rightarrow 0 \quad 0 \leqslant \mathrm{t} \leqslant \mathrm{T}
$$

Since $[\psi(t)]$ has been defined in equation (2.13) as a genuine solution, the condition for consistency may be modified by combining (2.12) with (2.13) to obtain 


$$
\left\|\frac{[\psi(t+\Delta t)]-[E(\Delta t)][\psi(t)]}{\Delta t}\right\|-0 \quad \text { as } \Delta t-0 \quad 0 \leqslant t \leqslant T
$$

where the quantity under the norm is sometimes called the TRUNCATION ERROR.

Equation (2.19) poses the condition for consistency in a form which allows one to examine the consistency of $[\mathrm{E}(\Delta \mathrm{t})]$ merely by taking a matrix power series of $[\mathrm{E}(\Delta \mathrm{t})]$ near $\mathrm{t}$ for small $\Delta \mathrm{t}$, and then comparing the expansion to a Taylor series expansion of $[\psi(t+\Delta t)]$.

To do this, one has first to write the expansion of $[\psi(t+\Delta t)]$ as

$$
[\psi(t+\Delta t)]=[\psi(t)]+\Delta t[\dot{\psi(t)}]+\frac{\Delta t^{2}}{2 !}[\ddot{\psi}(\dot{t})]+\frac{\Delta t^{3}}{3 !}[\ddot{\psi}(\dot{t})]+\ldots
$$

However, since $[\dot{\psi}(\mathrm{t})]=[\mathrm{A}(\mathrm{t})][\psi(\mathrm{t})]$, equation (2.15) may be expressed exactly as

$$
[\psi(t+\Delta t)]=\left\{[I]+\Delta t[A(t)]+\frac{\Delta t^{2}}{2 !}\left([A(t)]^{2}+[A(t)]\right)+\ldots\right\}[\psi(t)]
$$

providing $[A(t)]$ is analytic. If one substitutes (2.16) into equation (2.14), it should become apparent that the requirement for consistency here is that matrix power series expansion of $[\mathrm{E}(\Delta \mathrm{t})]$ agree at least through linear terms with the expansion on the right-hand side of equation (2.16).

Clearly, all Padé operators of the form $E_{p, q}(\Delta t[A])$ for which $p+q>0$ are by definition consistent approximations for the initial value problem posed by equation (1.28). In particular, for small $\Delta t$, $\mathrm{E}_{2,0}(\Delta \mathrm{t}[\mathrm{A}])$ may be expanded as 


$$
\mathrm{E}_{2,0}(\Delta \mathrm{t}[\mathrm{A}])=[\mathrm{I}]+\Delta \mathrm{t}[\mathrm{A}]+\frac{\Delta \mathrm{t}^{2}}{2}[\mathrm{~A}]^{2}+\mathrm{h}_{\mathrm{o}} \mathrm{O} . \mathrm{t}
$$

For the general case, where [A] is itself a function of time, and the constant $-[A]$ approximation $[\bar{A}]=\frac{1}{2}[[A(t)]+[A(t+\Delta t)]]$ is adopted, one may express the series as

$$
E_{2,0}(\Delta t[A])=[I]+\Delta t[A(t)]+\frac{\Delta t^{2}}{2}\left[\left[A(t)^{2}\right]+[A(t)]\right]+\text { h. o. t. }
$$

Comparing (2.18) with (2.16), one finds that the approximation $\mathrm{E}_{2,0}(\Delta \mathrm{t}[\overline{\mathrm{A}}])$ is indeed a consistent approximation for the initial value problem $(1.28)$.

For the $E_{1,1}^{\prime}(\Delta t[A])$ approximation, it is observed that for small enough $\Delta t$ :

$$
\begin{aligned}
E_{1,1}^{\prime}(\Delta t[A])= & {[I]+\frac{\Delta t}{2}[A(t)]+\frac{\Delta t}{2}[A(t+\Delta t)] } \\
& +\frac{\Delta t^{2}}{4}[A(t+\Delta t)][A(t)] \\
& +\frac{\Delta t^{2}}{4}[A(t+\Delta t)]^{2}+\text { h. o.. t. }
\end{aligned}
$$

Then, since $[A(t+\Delta t)]=[A(t)]+\Delta t[A \dot{(t)}]+\frac{\Delta t^{2}}{2}[\ddot{A}(\dot{t})]+\ldots$ one may write

$$
E_{1,1}^{\prime}(\Delta t[A])=[I]+\Delta t[A(t)]+\frac{\Delta t^{2}}{2}\left[[A(t)]^{2}+[\dot{A}(t)]\right]+\text { h. o.t. }
$$

which agrees through quadratic terms with the expansion given by (2.16). 
This establishes $E_{1,1}^{\prime}(\Delta t[A])$ as a consistent approximation to the initial value problem of $(1.28)$.

As a final point in this discussion of consistency, it is recalled that a necessary condition for the existence of the expansion given by (2.16) is that the matrix $[A(t)]$ must be analytic. Clearly, this requirement also holds for the expansions (2.18) and (2.19). Thus, in situations where the elements of the matrix [A(t)] undergo a step change, one must formally halt the consideration of the problem at that instant and begin to consider a new initial value problem defined in terms of the properties of the matrix $[A(t)]$ after the step change.

Given, then, that the two approximations of interest are consistent approximations, it would be valuable if one could next assume that after $N$ operations on the initial value function $\left[\psi_{O}\right]$ the discrete solution $[E(\Delta t)]^{N}\left[\psi_{0}\right]$ will approximate the exact solution $[\psi(N \Delta t)]$ to an even closer degree as $\Delta t$ shrinks in size and larger numbers of operations are required to "step out" in time to a fixed t. If this occurs, one may then say that the operator $[E(\Delta t)]$ provides a convergent approximation to the initial value problem.

Unfortunately, it cannot be assumed that $\mathrm{E}_{2,0}(\Delta \mathrm{t}[\mathrm{A}])$ and $\mathrm{E}_{1,1}^{\prime}(\Delta \mathrm{t}[\mathrm{A}])$ are convergent approximations simply because they are consistent. However, by using a theorem due to Lax, ${ }^{45}$ one may establish the conditions under which the approximations are convergent. Lax's theorem states that if the initial value problem is properly posed and a finite-difference approximation to it is made which satisfies the consistency condition, then stability is the necessary and sufficient condition for convergence.

To show that the initial value problem $(1.28)$ is properly posed, 
one must establish that (1) even though a genuine solution may not exist for some choice of initial element $\left[\psi_{0}\right]$ in the $\mathscr{B}$ space, it is possible to approximate this $\left[\psi_{0}\right]$ as closely as one wishes by another $\left[\psi_{0}^{i}\right]$ for which a genuine solution does exist, and that (2) the solution of (1.28) depends continuously on the initial data. It will now be assumed that the first of these two conditions does exist. The second condition will be met if the operator $[A(t)]$ is bounded, i. e., if $\|A(t)\|<K$ for $0 \leqslant t \leqslant T$.

The criteria for the boundedness of $[A(t)]$ stems from the definition, given in Chapter $I$, of the matrix $[\Lambda]$ which appears in $[A(t)]$. Since $[\Lambda]$ is inverted, care must be taken to avoid using linearly dependent trial functions in the modal expansion of $[\Phi(\vec{r}, t)]$, thereby making $[\Lambda]$ singular.

If this criteria is met, equation (1.28) may be said to be properly posed and the issue of convergence hangs on stability. The concern here is that there should be some limit on the extent to which any component of an initial function can be amplified as one steps out in time from $t=0$ to $t=T$. Since this numerical procedure involves a sequence of operations which approach infinity as $\Delta t$ approaches zero, the requirement for stability may be expressed as

DEFINITION 2.2. An approximation $[\mathrm{E}(\Delta \mathrm{t})]$ is said to be stable if, for some $\tau>0$, the $\infty$ set of operators

$$
\begin{array}{ll}
{[\mathrm{E}(\Delta \mathrm{t})]^{\mathrm{n}}} & 0<\Delta \mathrm{t}<\tau \\
& 0 \leqslant \mathrm{n} \Delta \mathrm{t} \leqslant \mathrm{T}
\end{array}
$$

is uniformly bounded. 
$\operatorname{Varga}^{42}$ points out that this condition is clearly met if the spectral radius of $[E(\Delta t)]$ is $<1$ for all $t>0$. In some problems, however, it is possible for a component of the exact solution to grow exponentially. In such cases (for example, a supercritical reactor) taking the spectral radius of $[\mathrm{E}(\Delta \mathrm{t})]<.1$ as a requirement is too stringent; and, in fact, its use would violate consistency.

Fortunately, a less stringent requirement for stability exists. Richtmyer and Morton ${ }^{46}$ point out that if, for some $G$ and some $T>0$

$$
\|\mathrm{E}(\Delta \mathrm{t})\| \leqslant 1+\mathrm{G} \Delta \mathrm{t} \quad \text { for } 0<\Delta \mathrm{t}<\tau
$$

then stability is guaranteed, for then $\|\mathrm{E}(\Delta t)\|^{\mathrm{n}} \leqslant \exp (G T)$, for $0 \leqslant$ $n \Delta t \leqslant T$.

For small enough $\Delta t$, it is now clear that both the $E_{2,0}(\Delta t[A])$ and $E_{1,1}^{\prime}(\Delta t[A])$ approximations are stable. To see this, one has only to recall that either approximation may be written as

$$
[E(\Delta t)]=[I]+\Delta t[A]+\text { h.o.t. }
$$

Obviously, if the elements of [A] are bounded, a sufficiently small $\Delta t$ exists such that

$$
\|\mathrm{E}(\Delta \mathrm{t})\|=\|[\mathrm{I}]+\Delta \mathrm{t}[\mathrm{A}]+\text { h. o.t. } \| \leqslant \mathrm{G} \Delta \mathrm{t}+\mathrm{l}
$$

The stability of the $\mathrm{E}_{2,0}(\Delta \mathrm{t}[\mathrm{A}])$ and $\mathrm{E}_{1,1}^{\prime}(\Delta \mathrm{t}[\mathrm{A}])$ approximations is thus assured for small enough $\Delta t$. There remains, however, one final topic to be considered in this discussion of the properties of these two approximations. The idea is somewhat akin to that of asymptotic stability. Stated roughly, it is that in obtaining an approximate solution, one would like to minimize the influence of those eigenvalues of 
$\mathrm{E}_{2,0}(\Delta \mathrm{t}[\mathrm{A}])$ and $\mathrm{E}_{1,1}^{\prime}(\Delta \mathrm{t}[\mathrm{A}])$ which correspond to eigenfunctions that do not approximate closely a genuine solution of the initial value problem. The question raised now is not so much what ahppens as $\Delta t \rightarrow 0$ but, rather, how accurate are the approximations for economically viable time step sizes.

To deal with this issue in a more precise way, the following theorem (established by Frobenius in 1878) is introduced: If $\lambda_{1}, \lambda_{2}, \ldots \lambda_{n}$ are the characteristic roots, distinct or not, of an $n \times n$ matrix [A], and if $G([A])$ is any polynomial function of $[A]$, then the characteristic roots of $G([A])$ are $G\left(\lambda_{1}\right), G\left(\lambda_{2}\right), \ldots G\left(\lambda_{n}\right)$.

The application of this theorem to the $\mathrm{E}_{2,0}(\Delta \mathrm{t}[\mathrm{A}])$ approximation is straightforward. By inspection, it is clear that the eigenvalues of $\mathrm{E}_{2,0}(\Delta \mathrm{t}[\overline{\mathrm{A}}])$ are given by $\frac{1}{1-\Delta \mathrm{t} \lambda_{i}+\frac{\Delta t}{2} \lambda_{i}^{2}}$, where $\lambda_{i}$ is an eigenvalue of $[\bar{A}]$.

In the case of the $E^{\prime}, 1,1$ (At]) approximation, however, one is confronted with the fact that the eigenvalues and eigenvectors of $\left[A\left(t_{p}+\Delta t\right)\right]$ are not the same as those of $\left[A\left(t_{p}\right)\right]$. Consequently, one may not, by inspection, claim that the eigenvalues of $E_{1,1}^{\prime}(\Delta t[A])$ are given by

$$
\frac{1+\frac{\Delta t}{2} \lambda_{i, o}}{1-\frac{\Delta t}{2} \lambda_{i, 1}}
$$

where $\lambda_{i, 0}$ is an eigenvalue of $\left[A\left(t_{p}\right)\right]$ and $\lambda_{i, l}$ is an eigenvalue of $\left[A\left(t_{p}+\Delta t\right)\right]$. Yet, in a qualitative sense, it can be maintained that since the parameters of $[A(t)]$ do not change very much over a time step for computationally realistic choices of $\Delta t$, the eigenvectors associated 
with $\left[A\left(t_{p}+\Delta t\right)\right]$ are not very different from those associated with $\left[A\left(t_{p}\right)\right]$. Given that this perturbation is slight, it then seems reasonable for one to conclude that within reasonably close bounds the eigenvalues of $E_{1,1}(\Delta t[A])$ may be approximated by (2.22).

These observations enable one to glean at least some qualitative information about the two approximations, $E_{2,0}(\Delta t[A])$ and $E_{1,1}^{\prime}(\Delta t[A])$. First, it seems clear that $\Delta t$ must be chosen so that the denominators $\left(1+\Delta t_{i}+\frac{\Delta t}{2} \lambda_{i}^{2}\right)$ and $\left(1-\frac{\Delta t}{2} \lambda_{i, 1}\right)$ do not equal 0 . Furthermore, one may now directly approach the question posed earlier of how to minimize the influence of those eigenvalues of $E_{2,0}(\Delta t[A])$ and $E_{1,1}^{\prime}(\Delta t[A])$ which corrupt the approximate solution. To do this, however, one must obtain some knowledge about the eigenvalue spectrum of the matrix $[A(t)]$.

For the quite stringent case of a symmetric reactivity matrix, $[\rho(t)]$, and constant, positive definite matrices $[\Lambda]$ and $\left[\beta_{i}\right]$, Porshing 47 has analyzed the eigenvalue spectrum of $[A(t)]$ in detail. Unfortunately, this kind of analysis has not been accomplished as yet for more general situations.

In lieu of this, one might rely on Porsching's results to gain some intuitive feeling for the spectrum of $[\mathrm{A}]$. For example, it can be maintained that at least one of the eigenvalues of [A] will be large and negative and that (even though the associated eigenfunction is dying away rapidly) this eigenvalue will influence the approximation in a detrimental way. It is then appropriate to turn to the $\mathrm{E}_{2,0}(\Delta \mathrm{t}[\mathrm{A}])$ approximation to ameliorate this situation. If $\lambda_{i}$ and $\lambda_{0}$ are two different eigenvalues of $[\bar{A}]$, and if $\left|\lambda_{i}\right| \gg\left|\lambda_{0}\right| i>0$, then for the $E_{2,0}(\Delta t[A])$ approximation 
$\left|\mathrm{E}_{2,0}\left(\Delta \mathrm{t} \lambda_{\mathrm{o}}\right)\right| \gg\left|\mathrm{E}_{2,0}\left(\Delta \mathrm{t} \lambda_{\mathrm{i}}\right)\right|$. It can consequently be argued that the influence of that large root on the solution is diminshed, thereby establishing further the motivation for one's use of $E_{2,0}(\Delta t[A])$ as an approximation to the exponential.

The whole series of $E_{p, q}(\Delta t[A])$ approximations, however, suffer to some extent from the necessity of assuming [A] constant over a time step. Da Nobrega ${ }^{48}$ has shown that if one uses $[\bar{A}]=\frac{1}{2}\left[\left[A\left(t_{p}\right)\right]+\right.$ $\left.\left[A\left(t_{p}+\Delta t\right)\right]\right]$, the approximation is automatically limited to a global error of $\mathscr{O}\left(\Delta \mathrm{t}^{2}\right)$. Moreover, this assumption, when used in any of the $E_{p, q}(\Delta t[A])$ approximations to the exponential, results in the reactor model responding to a ramp reactivity insertion by considering it to be a series of steps, each having its own small prompt jump. This produces a scalloped effect in the solution; and, in turn, motivates one to turn to the $E_{1,1}^{1}(\Delta t[A])$ approximation in an effort to handle ramp insertions better. This is really just a modification of the CrankNicholson ${ }^{49}$ method which may be thought of as imposing the requirement that the forward derivative of the solution at $t_{p}$ meet the backward derivative of the solution at $t_{p}+\Delta t$ at a point halfway through the time step (at $\left.t=\frac{\left(t_{p}+\Delta t\right)-t_{p}}{2}\right)$.

Finally, it should be pointed out that, although Porsching ${ }^{29}$ has directly applied the use of rational approximations of the Pade type successfully to the point-kinetics equations, the direct application of $\mathrm{E}_{2,0}(\Delta \mathrm{t}[\mathrm{A}])$ and $\mathrm{E}_{1,1}^{\prime}(\Delta \mathrm{t}[\mathrm{A}])$ to the multimode kinetics equations appears quite unattractive since it would require, at each time step, the inversion of $\left[\mathrm{I}-\Delta \mathrm{t}[\overline{\mathrm{A}}]+\frac{\Delta t}{2}[\overline{\mathrm{A}}]^{2}\right]$ or $\left[\mathrm{I}-\frac{\Delta \mathrm{t}}{2}\left[\mathrm{~A}\left(\mathrm{t}_{\mathrm{p}}+\Delta \mathrm{t}\right)\right]\right]$. Here 
[A] may range from order $(\mathrm{K} \times(1+\mathrm{I}))^{2}$ to order $(\mathrm{K} \times \mathrm{G} \times(1+\mathrm{I}))^{2}$ depending on whether or not the groups are collapsed. (Again, $\mathrm{K}=$ number of trial functions; $G=$ number of groups; $I=$ number of delayed groups.)

To get around this requirement, one would like to find (generalizing the work of da Nobrega ${ }^{34}$ ) an analytic inversion for these two matrices. The remainder of this chapter will describe such a generalization and apply the result to the $E_{2,0}(\Delta t[A])$ and $E_{1,1}^{\prime}(\Delta t[A])$ approximations.

\subsection{Development of an Analytic Inversion of $[[\mathrm{I}]-\delta[\mathrm{A}]]$}

Before attempting to find an analytic inversion for $\left[[\mathrm{I}]-\Delta \mathrm{t}[\overline{\mathrm{A}}]+\frac{\Delta \mathrm{t}}{2}[\overline{\mathrm{A}}]^{2}\right]$ and $\left[[I]-\frac{\Delta t}{2}\left[A\left(t_{p}+\Delta t\right)\right]\right]$, it should be noted that both matrices really involve inversions of a matrix of the form $[[I]-\delta[A]]$, where $\delta$ is some number (possibly complex). This is obvious in the case of $\left[[\mathrm{I}]-\frac{\Delta t}{2}\left[\mathrm{~A}\left(\mathrm{t}_{\mathrm{p}}+\Delta \mathrm{t}\right)\right]\right]$; but it is also true for $\left[[\mathrm{I}]-\Delta \mathrm{t}[\mathrm{A}]+\frac{\Delta \mathrm{t}}{2}[\mathrm{~A}]^{2}\right]$ since this matrix may be factored into $\{[\mathrm{I}]-\bar{\delta}[\mathrm{A}]][[\mathrm{I}]-\delta[\mathrm{A}]]\}^{-1}=[[\mathrm{I}]-\delta[\mathrm{A}]]^{-1}[[\mathrm{I}]-\bar{\delta}[\mathrm{A}]]^{-1}$, where $\delta \equiv$ $\frac{\Delta t}{2}(1-i)$ and $\bar{\delta} \equiv \frac{\Delta t}{2}(1+i)$. This section, then, is concerned with inverting $[[I]-\delta[A]]$ where $[A]$ is a matrix of the form given by equation $(1.28)$ and $\delta$ is a constant, either real or imaginary.

This inversion may be obtained in a straightforward manner by considering the matrix [A] with I delayed neutron groups. In this case, $[[\mathrm{I}]-\delta[\mathrm{A}]]$ may be written as 
$[[\mathrm{I}]-\delta[\mathrm{A}]]=\left[\begin{array}{cccc}{[\mathrm{I}]-\delta[\Lambda]^{-1}[\rho-\beta]} & -\lambda_{i}[\mathrm{I}] & \cdots & -\delta \lambda_{\mathrm{I}}[\mathrm{I}] \\ -\delta\left[\mu_{i}\right] & \left(1+\delta \lambda_{i}\right)[\mathrm{I}] & \\ \vdots & 0 & \ddots & 0 \\ -\delta\left[\mu_{I}\right] & & & \left(1+\delta \lambda_{I}\right)[\mathrm{I}]\end{array}\right]$

(2. 23)

where

$$
\left[\mu_{i}\right] \equiv[\Lambda]^{-1}\left[\beta_{i}\right]
$$

The $(I+I) \times(I+1)$ unknown matrix elements of $[[I]-\delta[A]]^{-1}$ may be found by using the general rule of partitioned matrices to solve

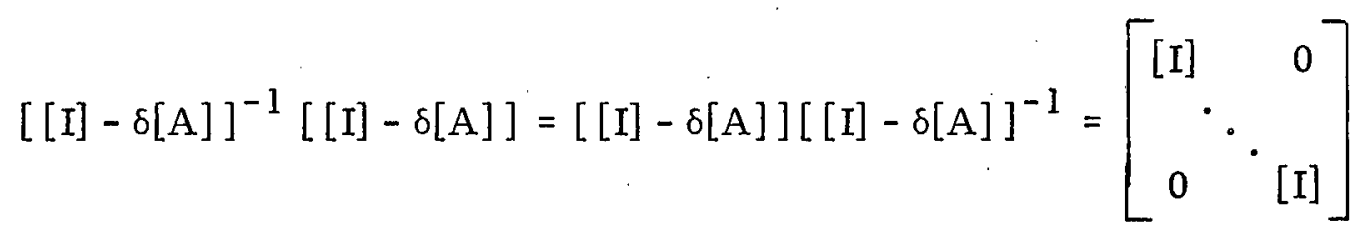

Equation (2.24) represents $(I+1) \times(I+1)$ matrix equations in $(I+1) \times$ (I+1) matrix unknowns. The unknown matrices may then be determined.

The result, which can be verified by substitution, can be written as

$$
[\mathrm{I}-\delta \mathrm{A}]^{-1}=\left\{\left[\begin{array}{c}
{[\mathrm{I}]} \\
\frac{1}{\epsilon+\lambda_{1}}\left[\mu_{1}\right] \\
\vdots \\
\frac{1}{\epsilon+\lambda_{\mathrm{I}}}\left[\mu_{\mathrm{T}}\right]
\end{array}\right]\left[\begin{array}{c}
{[\mathrm{I}] \frac{\lambda_{1}}{\epsilon+\lambda_{1}}[\mathrm{I}] \ldots \frac{\lambda_{\mathrm{I}}}{\epsilon+\lambda_{\mathrm{I}}}[\mathrm{I}]} \\
\end{array}\right\}\left[\delta^{-1}\right]+\right.
$$


$+\left[\begin{array}{cccc}0 & & \epsilon & 0 \\ & \frac{\epsilon+\lambda_{1}}{\epsilon+\mathrm{I}]} & \ddots & 0 \\ 0 & & & \frac{\epsilon}{\epsilon+\lambda_{I}}[I]\end{array}\right]$

where

$$
\left[\gamma^{-1}\right] \equiv\left[\begin{array}{ccc}
{[\gamma]^{-1}} & & 0 \\
& \ddots & \\
0 & & {[\gamma]^{-1}}
\end{array}\right] ; \quad \epsilon=\frac{1}{\delta}
$$

and

$$
[\gamma]^{-1}=\left[[I]-\delta[\Lambda]^{-1}[\rho]+\sum_{i}^{I} \frac{1}{\epsilon+\lambda_{i}}\left[\mu_{i}\right]\right]^{-1}
$$

If one then makes the following definitions:

$$
\begin{aligned}
& {[\mathrm{U}] \equiv \operatorname{col} .\left\{[\mathrm{I}] \frac{1}{\epsilon+\lambda_{1}}\left[\mu_{1}\right] \ldots \frac{1}{\epsilon+\lambda_{I}}\left[\mu_{\mathrm{I}}\right]\right\}} \\
& {[\mathrm{V}]^{\mathrm{T}} \equiv\left\{[\mathrm{I}] \frac{\lambda_{1}}{\epsilon+\lambda_{1}}[\mathrm{I}] \ldots \frac{\lambda_{\mathrm{I}}}{\epsilon+\lambda_{\mathrm{I}}}[\mathrm{I}]\right\}} \\
& {[\mathrm{D}] \equiv\left[\begin{array}{ccc}
0 & \frac{\epsilon}{\epsilon+\lambda_{1}}[\mathrm{I}] & 0 \\
0 & \ddots & \frac{\epsilon}{\epsilon+\lambda_{I}}[\mathrm{I}]
\end{array}\right]}
\end{aligned}
$$


equation (2.25) may be written as

$$
[[\mathrm{I}]-\delta[\mathrm{A}]]^{-1}=[\mathrm{U}][\mathrm{V}]^{\mathrm{T}}\left[\gamma^{-1}\right]+[\mathrm{D}]
$$

Thus, providing $[\gamma]^{-1}$ exists, an analytic inversion of the matrix $[[\mathrm{I}]-\delta] \mathrm{A}]]^{-1}$ does exist and is given by (2.27). What must now be done is to apply this inversion to the problem at hand, namely the solution of

$$
\left[\psi_{\mathrm{p}+1}\right]=\mathrm{E}_{2,0}(\Delta \mathrm{t}[\mathrm{A}])\left[\psi_{\mathrm{p}}\right] \text { and }\left[\psi_{\mathrm{p}+1}\right]=\mathrm{E}_{1,1}^{\mathrm{j}}(\Delta \mathrm{t}[\mathrm{A}])\left[\psi_{\mathrm{p}}\right]
$$

It is the purpose of the next two sections to accomplish this task.

$$
\text { 2.4 Application of the Analytic Inversion to }
$$

This section begins with the substitution of equation (2.27) into

$$
\mathrm{E}_{2,0}(\Delta \mathrm{t}[\mathrm{A}])=[[\mathrm{I}]-\delta[\mathrm{A}]]^{-1}[[\mathrm{I}]-\bar{\delta}[\mathrm{A}]]^{-1}
$$

In this way, it is discovered that

$$
\begin{aligned}
\mathrm{E}_{2,0}(\Delta \mathrm{t}[\mathrm{A}])= & {[\mathrm{U}][\mathrm{V}]^{\mathrm{T}}\left[\gamma^{-1}\right][\overline{\mathrm{U}}][\overline{\mathrm{V}}]^{\mathrm{T}}\left[\gamma^{-1}\right]+[\mathrm{D}][\overline{\mathrm{D}}] } \\
& +[\mathrm{U}][\mathrm{V}]^{\mathrm{T}}\left[\gamma^{-1}\right][\overline{\mathrm{D}}]+[\mathrm{D}][\overline{\mathrm{U}}][\overline{\mathrm{V}}]^{\mathrm{T}}\left[\bar{\gamma}^{-1}\right]
\end{aligned}
$$

where the bars denote complex conjugates of the matrices defined by (2. 26).

In spite of the emergence of complex constants in (2.28) it should be clear that $E_{2,0}(\Delta t[A])$ is real, since $E_{2,0}(\Delta t[A])=[[I]-\Delta t[A]+$ $\left.\frac{\Delta t^{2}}{2}[A]^{2}\right]^{-1}$ and $[A]$ is real. This leads one to note that if the right- 
hand side of $(2.28)$ may be factored into its real and imaginary parts, yielding

$$
E_{2,0}(\Delta t[A])=[R E A L]+[\text { IMAG }]
$$

it then follows that the imaginary part, [IMAG], must equal the null matrix [0]. One is thus motivated to reform (2.28) into (2.29) and thereby eliminate the imaginary part of the matrix.

At this point, the following definitions are introduced:

$$
\begin{aligned}
& {[\mathrm{D}] \equiv\left[\mathrm{D}_{1}\right]+i\left[\mathrm{D}_{2}\right] \quad[\mathrm{V}]^{\mathrm{T}} \equiv\left[\mathrm{V}_{1}\right]^{\mathrm{T}}-\mathrm{i}\left[\mathrm{V}_{2}\right]} \\
& {[\mathrm{U}] \equiv\left[\mathrm{U}_{1}\right]-\mathrm{i}\left[\mathrm{U}_{2}\right] \quad\left[\gamma^{-\mathrm{l}}\right] \equiv[[\mathrm{a}]+[\mathrm{b}] \mathrm{i}]}
\end{aligned}
$$

where

$$
\begin{aligned}
& {[\mathrm{a}] \equiv\left[\mathrm{I}-\frac{\Delta \mathrm{t}}{2}[\Lambda]^{-1}[\rho]+\underset{i}{\sum_{i}} \Delta \mathrm{tP}_{i}\left(1+\Delta t \lambda_{i}\right)\left[\mu_{i}\right]\right]} \\
& {[\mathrm{b}] \equiv\left[\frac{\Delta \mathrm{t}}{2}[\Lambda]^{-1}[\rho]-\sum_{i} \Delta t P_{i}\left[\mu_{i}\right]\right]}
\end{aligned}
$$

and

$$
P_{i} \equiv \frac{1}{\left(1+\Delta t \lambda_{i}\right)^{2}+1}
$$

Using these definitions, equation (2.28) may now be expanded to yield the following: 


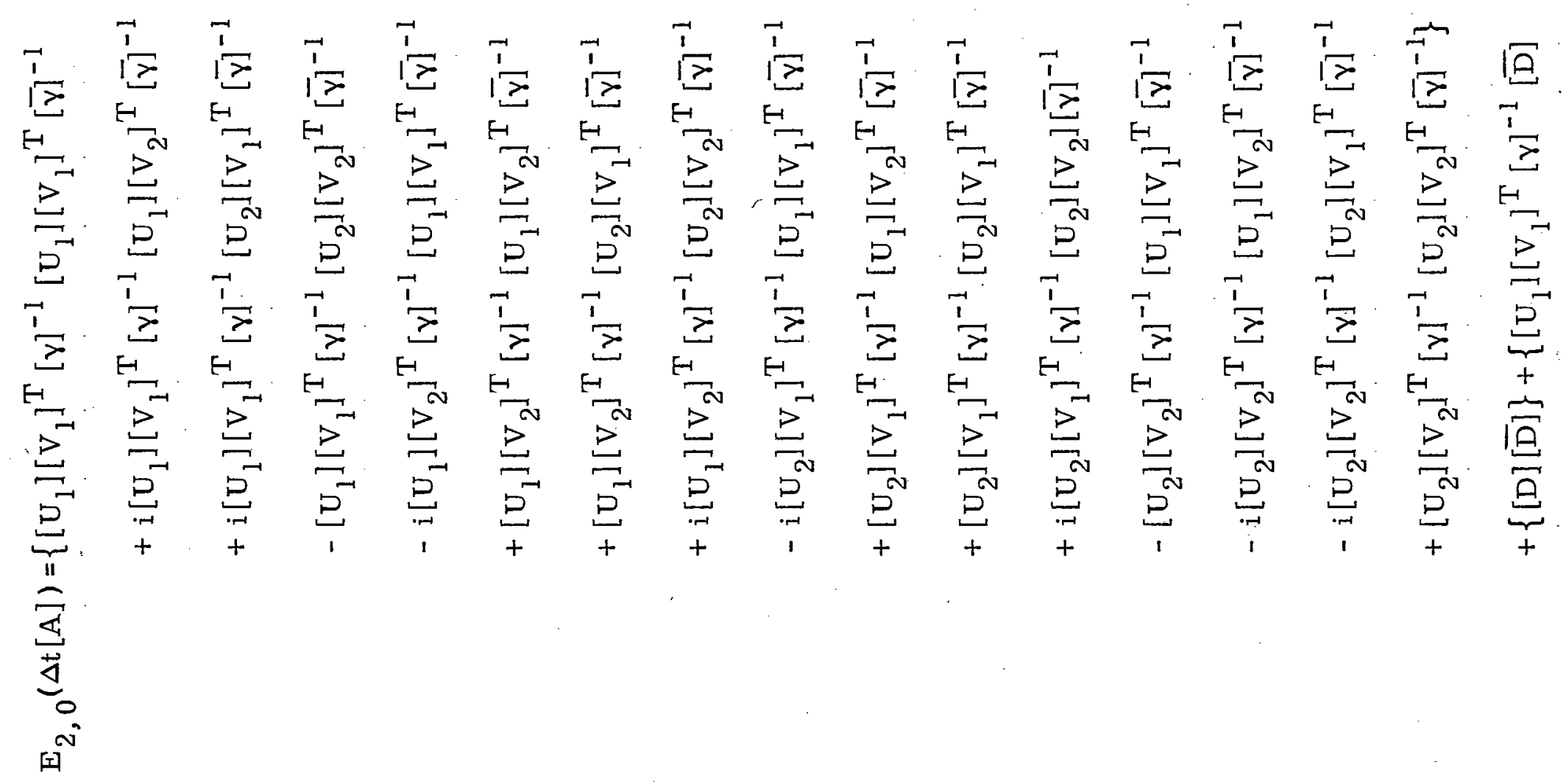




$$
\begin{aligned}
& -i\left[U_{1}\right]\left[v_{2}\right]^{T}[\gamma]^{-1}[\bar{D}]-i\left[U_{2}\right]\left[v_{1}\right]^{T}[\gamma]^{-1}[\bar{D}] \\
& \left.-\left[U_{2}\right]\left[v_{2}\right]^{T}[\gamma]^{-1}[\bar{D}]\right\}+\left\{[D]\left[U_{1}\right]\left[v_{1}\right]^{T}[\bar{\gamma}]^{-1}\right. \\
& +i[D]\left[U_{1}\right]\left[v_{2}\right]^{T}[\bar{\gamma}]^{-1}+i[D]\left[U_{2}\right]\left[v_{1}\right]^{T}[\bar{\gamma}]^{-1} \\
& \left.-[D]\left[U_{2}\right]\left[V_{2}\right]^{T}[\bar{\gamma}]^{-1}\right\}
\end{aligned}
$$

The motivation for eliminating the imaginary parts of $E_{2,0}(\Delta t[A])$ is now stronger than ever. To do this, however, requires that the imaginary and real parts of equation (2.20) be segregated. Accordingly, the matrices $[Z]$ and $[E]$ are defined in the following way: If one writes

$$
[\gamma]^{-1}=[[a]+i[b]]^{-1}=[z]+i[e]
$$

it can be shown that $[e]=\left[a b^{-1} a+b\right]^{-1}$ and $[z]=\left[b^{-1} a e\right]=\left[b a^{-1} b+a\right]^{-1}$. (The proof is given in Appendix I.) [Z] is then defined as

$$
\left[\begin{array}{ccc}
{[z]} & & 0 \\
& \ddots & \\
0 & & {[z]}
\end{array}\right]
$$

and $[\mathrm{E}]$ is defined as

$$
\left[\begin{array}{ccc}
{[\mathrm{e}]} & & 0 \\
& \ddots & \\
0 & & {[\mathrm{e}]}
\end{array}\right]
$$

Finally, if $\left[\mathrm{M}_{\mathrm{ij}}\right] \equiv\left[\mathrm{U}_{\mathrm{i}}\right]\left[\mathrm{V}_{\mathrm{j}}\right]^{\mathrm{T}}$ and $[\mathrm{L}] \equiv[\mathrm{D}][\overline{\mathrm{D}}]$, then equation (2.31) may be written in the form of (2.29), where 


$$
\begin{aligned}
& {[\text { IMAG }]=\left\{\left[\mathrm{M}_{11}\right][\mathrm{E}]+\left[\mathrm{M}_{12}\right][\mathrm{Z}]+\left[\mathrm{M}_{21}\right][\mathrm{Z}]-\left[\mathrm{M}_{22}\right][\mathrm{E}]\right\} } \\
& \cdot\left\{-\left[\mathrm{M}_{11}\right][\mathrm{Z}]+\left[\mathrm{M}_{12}\right][\mathrm{E}]+\left[\mathrm{M}_{21}\right][\mathrm{E}]+\left[\mathrm{M}_{22}\right][\mathrm{Z}]\right\} \\
&-\left\{-\left[\mathrm{M}_{11}\right][\mathrm{Z}]+\left[\mathrm{M}_{12}\right][\mathrm{E}]+\left[\mathrm{M}_{21}\right][\mathrm{E}]+\left[\mathrm{M}_{22}\right][\mathrm{Z}]\right\} \\
& \cdot\left\{\left[\mathrm{M}_{11}\right][\mathrm{E}]+\left[\mathrm{M}_{12}\right][\mathrm{Z}]+\left[\mathrm{M}_{21}\right][\mathrm{Z}]-\left[\mathrm{M}_{22}\right][\mathrm{E}]\right\} \\
&-\left\{\left[\mathrm{M}_{11}\right][\mathrm{E}]+\left[\mathrm{M}_{12}\right][\mathrm{Z}]+\left[\mathrm{M}_{21}\right][\mathrm{Z}]-\left[\mathrm{M}_{22}\right][\mathrm{E}]\right\}\left[\mathrm{D}_{1}\right] \\
&+\left[\mathrm{D}_{1}\right]\left\{\left[\mathrm{M}_{11}\right][\mathrm{E}]+\left[\mathrm{M}_{12}\right][\mathrm{Z}]+\left[\mathrm{M}_{21}\right][\mathrm{Z}]-\left[\mathrm{M}_{22}\right][\mathrm{E}]\right\} \\
&+\left\{-\left[\mathrm{M}_{11}\right][\mathrm{Z}]+\left[\mathrm{M}_{12}\right][\mathrm{E}]+\left[\mathrm{M}_{21}\right][\mathrm{E}]+\left[\mathrm{M}_{22}\right][\mathrm{Z}]\right\}\left[\mathrm{D}_{2}\right] \\
&-\left[\mathrm{D}_{2}\right]\left\{-\left[\mathrm{M}_{11}\right][\mathrm{Z}]+\left[\mathrm{M}_{12}\right][\mathrm{E}]+\left[\mathrm{M}_{21}\right][\mathrm{E}]+\left[\mathrm{M}_{22}\right][\mathrm{Z}]\right\}
\end{aligned}
$$

and

$$
\begin{aligned}
{[\text { REAL }]=} & \left\{-\left[\mathrm{M}_{11}\right][\mathrm{Z}]+\left[\mathrm{M}_{12}\right][\mathrm{E}]+\left[\mathrm{M}_{21}\right][\mathrm{E}]+\left[\mathrm{M}_{22}\right][\mathrm{Z}]\right\}^{2} \\
& +\left\{\left[\mathrm{M}_{11}\right][\mathrm{E}]+\left[\mathrm{M}_{12}\right][\mathrm{Z}]+\left[\mathrm{M}_{21}\right][\mathrm{Z}]-\left[\mathrm{M}_{22}\right][\mathrm{E}]\right\}^{2} \\
& -\left\{-\left[\mathrm{M}_{11}\right][\mathrm{Z}]+\left[\mathrm{M}_{12}\right][\mathrm{E}]+\left[\mathrm{M}_{21}\right][\mathrm{E}]+\left[\mathrm{M}_{22}\right][\mathrm{Z}]\right\}\left[\mathrm{D}_{1}\right] \\
& -\left\{\left[\mathrm{M}_{11}\right][\mathrm{E}]+\left[\mathrm{M}_{12}\right][\mathrm{Z}]+\left[\mathrm{M}_{21}\right][\mathrm{Z}]-\left[\mathrm{M}_{22}\right][\mathrm{E}]\right\}\left[\mathrm{D}_{2}\right] \\
& -\left[\mathrm{D}_{1}\right]\left\{-\left[\mathrm{M}_{11}\right][\mathrm{Z}]+\left[\mathrm{M}_{12}\right][\mathrm{E}]+\left[\mathrm{M}_{21}\right][\mathrm{E}]+\left[\mathrm{M}_{22}\right][\mathrm{Z}]\right\} \\
& +\left[\mathrm{D}_{2}\right]\left\{\left[\mathrm{M}_{11}\right][\mathrm{E}]+\left[\mathrm{M}_{12}\right][\mathrm{Z}]+\left[\mathrm{M}_{21}\right][\mathrm{Z}]-\left[\mathrm{M}_{22}\right][\mathrm{E}]\right\}+[\mathrm{L}]
\end{aligned}
$$

$$
[\mathrm{L}]=\left[\begin{array}{cccc}
{[0]} & & & 0 \\
& 2 \mathrm{P}_{1}[\mathrm{I}] & & \\
& & \ddots & \\
0 & & & 2 \mathrm{P}_{\Upsilon}[\mathrm{I}]
\end{array}\right]
$$


In verifying that [IMAG] $=0$, two identities arise. (The proof of this is given in Appendix II.) Their existence stems from the way in which $[z]$ and $[e]$ were defined, and the fact that $[z]=[-e]+[b]^{-1}[e]-$ $\sum_{i=1}^{I} \Delta t^{2} P_{i} \lambda_{i}[b]^{-1}\left[\mu_{i}\right][e]$. The identities are

$$
\begin{gathered}
{[z][e]-[e][z]-\sum_{i=1}^{I} \Delta t^{2} P_{i} \lambda_{i}\left[[e]\left[\mu_{i}\right][z]\right.} \\
\left.-[z]\left[\mu_{i}\right][e]\right]=0
\end{gathered}
$$

and

$$
\begin{aligned}
& {[e]^{2}+[z]^{2}+\sum_{i=1}^{I} \Delta t^{2} P_{i} \lambda_{i}\left[[e]\left[\mu_{i}\right][e]\right.} \\
& \left.\quad+[z]\left[\mu_{i}\right][z]\right]=[e]+[z]
\end{aligned}
$$

Although the identities (2.34) and (2.35) may indeed be used to verify that [IMAG] $=[0]$, their real importance here lies in their use in the restructuring of [REAL] into a more tractable form. The result of doing this (see Appendix III) is that the matrix [REAL] may be written as

$$
\begin{aligned}
{[\mathrm{REAL}]=} & {\left.[\Lambda]+\left[\left[\mathrm{U}_{1}\right]\left[\mathrm{V}_{1}\right]^{\mathrm{T}}-\left[\mathrm{U}_{2}\right]\left[\mathrm{V}_{2}\right]^{\mathrm{T}}\right][\mathrm{Z}]+[\mathrm{E}]\right] } \\
& +\left[\left[\mathrm{U}_{1}\right]\left[\mathrm{V}_{2}\right]^{\mathrm{T}}+\left[\mathrm{U}_{2}\right]\left[\mathrm{V}_{1}\right]^{\mathrm{T}}\right][[\mathrm{Z}-\mathrm{E}]]
\end{aligned}
$$

And, since $[$ IMAG $]=[0]$, one finds that 


$$
\begin{aligned}
{\left[\psi_{p+1}\right]=} & \left\{[\Lambda]+\left[\left[\mathrm{U}_{1}\right]\left[\mathrm{V}_{1}\right]^{\mathrm{T}}-\left[\mathrm{U}_{2}\right]\left[\mathrm{V}_{2}\right]^{\mathrm{T}}\right][[\mathrm{Z}]+\lfloor\mathrm{E}]]\right. \\
& \left.+\left[\left[\mathrm{U}_{1}\right]\left[\mathrm{V}_{2}\right]^{\mathrm{T}}+\left[\mathrm{U}_{2}\right]\left[\mathrm{V}_{1}\right]^{\mathrm{T}}\right][[\mathrm{Z}]-[\mathrm{E}]]\right\}\left[\psi_{\mathrm{p}}\right]
\end{aligned}
$$

For the point-kinetics case, equation (2.37) is identical with the result given by Nóbrega. 34

Two final comments should be made concerning the result, given by (2.37), of applying the analyt1c inversion developed in the previous section to the equation $\left[\psi_{p+1}\right]=E_{2,0}(\Delta t[A])\left[\psi_{p}\right]$. First, for changes in $\Sigma_{a}$ (the poison capture cross section) only the block diagonal matrices $[Z]$ and $[E]$ change over $\Delta t$ since only these matrices contain the reactivity matrix $[\rho]$. Therefore, for transients for which the sole change is in $\Sigma_{a}$, only these matrices must be recomputed at each time step. The result is a substantial savings in computation time over the amount of work which would have been required in using [REAL] as expressed in equation (2.33). Second, although at each time step two matrices must be inverted, the matrices are quite small. The inversions occur when $[Z]$ and $[E]$ are generated and involve the inversion of $[b]$ and $\left[a b^{-1} \mathrm{a}+\mathrm{b}\right]$. If the number of trial functions in the modal approximation is $\mathrm{K}$ and $\mathrm{G}$ energy groups are considered, then the maximum size of these matrices is $(G \times K) \times(G \times K)$. For a fully collapsed approach, their size is $(K) \times(K)$.

2.5 Application of the Analytic Inversion to $\left[\psi_{\mathrm{p}+1}\right]=$

$$
\mathrm{E}_{\mathrm{p}+\mathrm{I}}^{\prime}(\Delta \mathrm{t}[\mathrm{A}])\left[\psi_{\mathrm{p}}\right]
$$

The application of the analytic inversion of $[[I]-\delta[A]]$ applies in a straightforward way to the $E_{1,1}^{\prime}(\Delta t[A])$ approximation. Since 
$E_{1,1}^{\prime}(\Delta t[A])=\left[[I]-\frac{\Delta t}{2}\left[A\left(t_{p}+\Delta t\right)\right]\right]^{-1}\left[[I]-\frac{\Delta t}{2}\left[A\left(t_{p}\right)\right]\right]$, one may substitute equation (2.25), with $\delta=\frac{\Delta t}{2}$, directly into $E_{1,1}^{\prime}(\Delta t[A])$ and obtain

$$
\begin{aligned}
E_{1,1}^{\prime}(\Delta t[A]) & =\left[\left\{[U][V]^{T}\right\}\left[\gamma^{-1}\right]+[D]\right]\left[[I]+\frac{\Delta t}{2}\left[A\left(t_{p}\right)\right]\right] \\
& \equiv[M 1]\left[[I]+\frac{\Delta t}{2}\left[A\left(t_{p}\right)\right]\right]
\end{aligned}
$$

where the elements of [M1] are defined by equation (2.26) and contain those properties associated with the matrix [A] at the end of the time step.

Applying equation $(2.38)$ to the matrix equation $\left[\psi_{p+1}\right]=$ $E_{1,1}^{\prime}(\Delta t[A])\left[\psi_{p}\right]$, yields

$$
\left[\psi_{p+1}\right]=\left[\left\{[\mathrm{U}][\mathrm{V}]^{\mathrm{T}}\right\}\left[\gamma^{-1}\right]+[\mathrm{D}]\right]\left[\left[\psi_{\mathrm{p}}\right]+\frac{\Delta \mathrm{t}}{2}\left[\mathrm{~A}\left(\mathrm{t}_{\mathrm{p}}\right)\right]\left[\psi_{\mathrm{p}}\right]\right]
$$

Again, it should be noted that for transients for which the sole change is in $\Sigma_{a}$, only the block diagonal matrix $\left[\gamma^{-1}\right]$ changes over a time step. Here, the situation is even simpler than for the $E_{2,0}(\Delta t[A]$ ) approximation. At each time step only one inversion must be made of $[\gamma]^{-1}$, a matrix whose size is either $(\mathrm{G} \times \mathrm{K}) \times(\mathrm{G} \times \mathrm{K})$ for an uncollapsed treatment or $(K) \times(K)$ for a collapsed treatment $(G \equiv$ number of groups; $\mathrm{K} \equiv$ number of trial functions).

In the past three sections, a generalization has been made of an analytic inversion technique, developed by da Nobrega ${ }^{34}$ for the pointkinetics matrix. It should now be clear that this generalization may be applied to the multimode kinetics equations when these equations are placed in a point-kinetics form. Specifically, this technique has been 
applied successfully to two consistent and stable approximations to the exponential, $\exp (\Delta t[A])$. Finally, the results of this application have been used to generate the $\left[\psi_{p+1}\right]$ vector of equations (1.29) from $\left[\psi_{p}\right]$, thus "stepping out in time" from $t_{p}$ to $t_{p+1}$. In the next chapter, several numerical studies will be presented which are intended to demonstrate the efficiency of this new approach (exemplified by equations (2.37 and (2.39)) when used to solve the multimode kinetics equations. 


\section{CHAPTER III \\ NUMERICAL RESULTS}

In Chapter I, a set of multimode kinetics equations were developed in a point-kinetics form by the application of time synthesis to the timedependent, multigroup diffusion equations. In Chapter II, it was then observed that one may solve these equations by using a temporal integration technique which utilizes the method of undetermined parameters. Alternatively, however, it was also found that one may approach the solution of these equations by generalizing the space-independent, pointkinetics work of da Nóbrega. ${ }^{39}$ This new approach, which involves the application of nonanalytic inversion of the matrix $[[\mathrm{I}]-\delta[A]]$ to "ratios" of matrix polynomials, was applied to the $\mathrm{E}_{2,0}(\Delta \mathrm{t}[\mathrm{A}])$ and $E_{1,1}^{\mathrm{R}}(\Delta \mathrm{t}[\mathrm{A}])$ approximations to the exponential, $\exp (\Delta \mathrm{t}[\mathrm{A}])$, of equation (1.29), and the results of that application were presented in equations (2.37) and (2.39). It is the purpose of the present chapter to investigate the efficiency of these results by considering several numerical examples.

To perform this investigation, two one-dimensional slab reactor models were studied. Both reactors were described by multimodal kinetics equations which had been derived by the application of time synthesis in a fully collapsed manner, using two trial functions. Thus, in these studies, the neutron flux was approximated by

$$
[\Phi(x, t)]=\left[\psi_{1}(x)\right] T_{1}(t)+\left[\psi_{2}(x)\right] T_{2}(t)
$$

where the vectors $\left[\psi_{1}(x)\right]$ and $\left[\psi_{2}(x)\right]$ were predetermined trial functions 
and the scalars $T_{1}(t)$ and $T_{2}(t)$ were unknown amplitude functions. For both slab reactors, the two trial functions were selected so as to "bracket" the transient being considered. To do this, the first trial function was picked to correspond to the initial value of the problem at hand (i。e., the steady-state neutron flux distribution of the unperturbed reactor). The second trial function then corresponds to the steady-state flux distribution of a pseudo-critical, perturbed reactor. This trial function was found for each transient by adjusting the number of neutrons produced per fission in order to make critical the material composition associated with the reactor at the end of the transient.

Also, for each slab, the weighting functions used were the adjoint functions calculated from the transpose of the operators associated with the two trial functions. Both the trial functions and the adjoints were calculated using a computer program, DIFFUSE, which was written principally by William Reed. 50

The first reactor considered has the same dimension and critical composition as the reactor considered by Fuller, Meneley, and Hetrick ${ }^{39}$ Furthermore, the analysis of this reactor paralleled the numerical work of these authors in that the neutronics of the reactor were described with one neutron energy group and one precursor group.

Three different reactıvity insertions were analyzed for this slab reactor: a large negative step insertion, a positive ramp insertion that became prompt critical, and a sub-prompt critical, positive step insertion. The spatial neutron distribution following each of these insertions was predicted as a function of time by both the temporal integration method presented by Fuller, Meneley, and Hetrick ${ }^{39}$ and 
by the new method that is the thrust of the present work.

Four computer programs were used to perform and double-check this analysis. The first, $\operatorname{MITIM}-\mathrm{E}(2,0)$, is a computer code based on the algorithm of equation (2.37). The second, $\operatorname{MITIM}-E^{\prime}(1,1)$, is a code based on the algorithm of equation (2.39). (Both MITIM-E $(2,0)$ and MITIM-E' $(1,1)$ are described in Appendix IV.) The third program, MOVER, was adopted directly from an advancement subroutine written by E. L. Fuller ${ }^{51}$ which utilizes the method of undetermined parameters in a temporal integration. Second degree, piecewise polynomials and subdomain weighting were used in the time integration. Finally, the fourth computer program, SPATKIN, acted as an independent check of the solutions for the multimode kinetics equations obtained by MITIME(2,0), MITIM-E' $(1,1)$, and by MOVER. This program was developed by da Nóbrega ${ }^{52}$ and utillzes the $\theta$-method to predict accurately the spatial distribution of the neutron population as a function of time.

The second slab reactor considered was described by a model composed of two neutron energy groups and six precursor groups. A positive ramp insertion was analyzed using MITIM-E $(2,0)$ and MITIM$E^{\prime}(1,1)$ and the results were compared to those obtained from GAKIN, a direct, one-dimensional, multigroup kinetics code developed by K. F : Hansen and S. R. Johnson. 53

The remainder of this chapter is a presentation of the results of these numerical studies. In section 3.1, the analysis of the first reactor is presented; the analysis of the second follows in section 3.2. 


\subsection{Reactor Number One - One-Group Results}

The first reactor considered was a $240-\mathrm{cm}$ slab with the critical parameters given in Table III-1. The mesh spacing; $\Delta \mathrm{x}$, is $4.0 \mathrm{~cm}$.

It was noted previously that the reactor was modeled with one precursor group. The delayed neutron fraction, $\beta$, was taken as 0.0064 and the precursor decay constant, $\lambda$, as $0.08 \mathrm{sec}^{-1}$.

\section{Case 1: Large Negative Reactivity Insertion}

The first of the three transients analyzed in this section was initiated by the sudden insertion of a neutron absorber into Region III of the slab reactor. This insertion amounts to a step change in the macro-

scopic absorption cross section, $\Sigma_{a}$, in Region III from $\Sigma_{a}=0.194962$ $\mathrm{cm}^{-1}$ to $0.021 \mathrm{~cm}^{-1}$.

Figure III-1 presents the trial and weight functions selected to bracket this transient. The amplitudes associated with the two trial functions are then separated at various times during the transient in Table III-2. These results are given for various selections of $\Delta t$, the size of the time step selected.

In Table III-2, the predictions of $\operatorname{MITIM-E}(2,0)$ and $\operatorname{MITIM-E}(1,1)$ are compared to those of two versions of MOVER. MOVER-I selects its own time step by requiring that the rate of growth of the amplitude functions be within a predetermined limit specified by the selection of: a parameter, $\epsilon$. MOVER-II, on the other hand, requires the user to select a priori the size of $\Delta t$. As a consequence of this requirement, MOVER-II provides greater utility than MOVER-I in comparing the 


\begin{tabular}{|c|c|c|c|c|c|}
\hline & & Paramet & $\begin{array}{l}\text { le III- } 1 \\
\text { s for Criticalit }\end{array}$ & & \\
\hline $\begin{array}{l}\text { Parameter } \\
\text { (units) }\end{array}$ & $\begin{array}{c}\text { Region I } \\
(0-60 \mathrm{~cm})\end{array}$ & $\begin{array}{c}\text { Region II } \\
(60-104 \mathrm{~cm})\end{array}$ & $\begin{array}{c}\text { Region III } \\
(104-136 \mathrm{~cm})\end{array}$ & $\begin{array}{c}\text { Region IV } \\
(136-180 \mathrm{~cm})\end{array}$ & $\begin{array}{c}\text { Region V } \\
(180-240 \mathrm{~cm})\end{array}$ \\
\hline$D(\mathrm{~cm})$ & 1.69531 & 1.69531 & 1.69531 & 1.69531 & 1.69531 \\
\hline$v \Sigma_{f}\left(\mathrm{~cm}^{-1}\right)$ & 0.0194962 & 0.194962 & 0.194962 & 0.194962 & 0.0194962 \\
\hline$\Sigma_{a}\left(\mathrm{~cm}^{-1}\right)$ & 0.0183343 & 0.194962 & 0.194962 & 0.194962 & 0.0183343 \\
\hline $\mathrm{v}(\mathrm{cm} / \mathrm{sec})$ & $10^{6}$ & $10^{6}$ & $10^{6}$ & $10^{6}$ & $10^{6}$ \\
\hline
\end{tabular}


Normalized Trial Function $\left(\psi_{k}(x)\right)$ and Weight Function $\left(\omega_{p}(x)\right)$

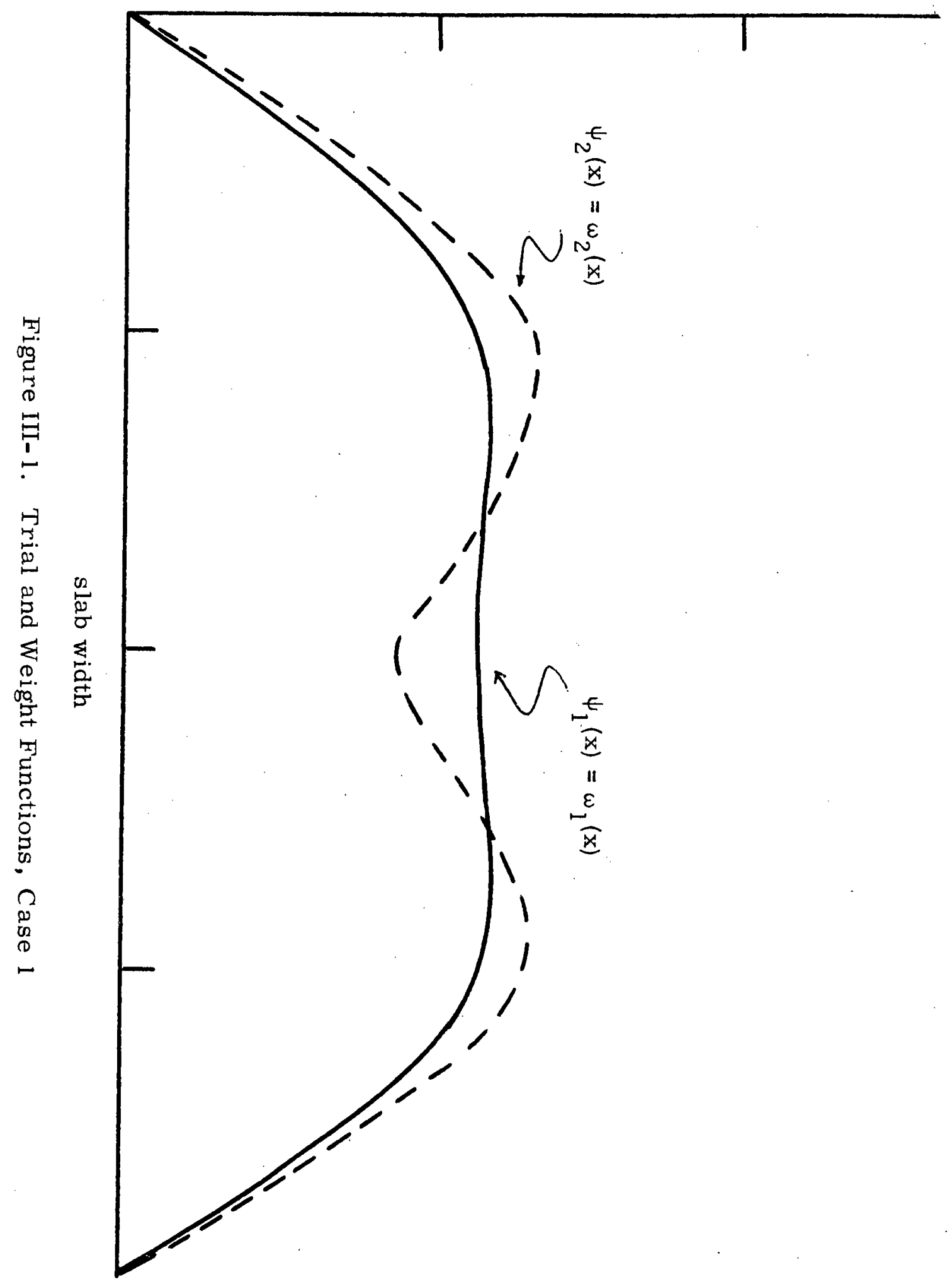




\begin{tabular}{|c|c|c|c|c|c|c|c|c|c|}
\hline \multirow[b]{2}{*}{ Time } & & \multicolumn{2}{|c|}{ MOVER I } & \multicolumn{2}{|c|}{ MOVER II } & \multicolumn{2}{|c|}{ MITIM-E $(2,0)$} & \multicolumn{2}{|c|}{ MITIM-E' $(1,1)$} \\
\hline & & $\epsilon=10^{-4}$ & $\epsilon=10^{-3}$ & $\Delta t=10^{-3}$ & $\Delta t=10^{-2}$ & $\Delta t=10^{-3}$ & $\Delta t=10^{-2}$ & $\Delta t=10^{-3}$ & $\Delta t=10^{-2}$ \\
\hline \multirow{2}{*}{0.0} & $\mathrm{~T}_{1}$ & 1.0 & 1.0 & 1.0 & 1.0 & 1.0 & 1.0 & 1.0 & 1.0 \\
\hline & $\mathrm{T}_{2}$ & 0.0 & 0.0 & 0.0 & 0.0 & 0.0 & 0.0 & 0.0 & 0.0 \\
\hline \multirow[b]{2}{*}{.003} & $\mathrm{~T}_{1}$ & $.0373^{*}$ & $.0373^{* * *}$ & .0378 & - & .0385 & - & .0263 & - \\
\hline & $\mathrm{T}_{2}$ & .5696 & .5700 & .5690 & - & .5715 & - & .5784 & - \\
\hline \multirow{2}{*}{.01} & $\mathrm{~T}_{1}$ & .0380 & .0380 & .0381 & .6976 & .0380 & .0397 & .0382 & -.811 \\
\hline & $\mathrm{T}_{2}$ & .3627 & .3627 & .3624 & -.2685 & .3637 & .4013 & .3619 & 1.03 \\
\hline \multirow{2}{*}{.03} & $\mathrm{~T}_{1}$ & .0381 & .0381 & .0381 & .3471 & .0381 & .0381 & .0381 & -.6212 \\
\hline & $\mathrm{T}_{2}$ & .3341 & .3391 & .3390 & .0334 & .3391 & .3397 & .3391 & .983 \\
\hline \multirow{2}{*}{.10} & $\mathrm{~T}_{1}$ & .0379 & .0379 & .0378 & .0607 & .0376 & .0380 & .0378 & .3094 \\
\hline & $\mathrm{T}_{2}$ & .3379 & .3379 & .3380 & .3153 & .3379 & .3379 & .3379 & .069 \\
\hline
\end{tabular}

*took 44 time steps to get to $t=.002934 \mathrm{sec}$ *** took 19 time steps to get to $t=.003098 \mathrm{sec}$ 
efficiency of the method in that it enables one to observe how the method fares under various choices of $\Delta t$, the size of the time step.

Finally, the results given in Table III-2 for MOVER-I were obtained for two values of epsilon. In order to compare these results easily with those of the other codes, the amplitude functions predicted by MOVER-I were linearly interpolated to correspond to the times shown.

When one considers the results of Table III-2, at least two observations may be made immediately. First, the rather qualitative motivation given in Chapter II for using the $E_{2,0}(\Delta t[A])$ approximation appears justified. The step insertion of a large amount of negative reactivity served in this case as a test of the ability of each method to deal with troublesome, large, negative eigenvalues of the matrix [A]. Table III-2 shows that the $\mathrm{E}_{2,0}(\Delta \mathrm{t}[\mathrm{A}])$ approximation, acting as the basis for MITIM-E(2,0), responded favorably to the test, for it shows that this approximation was more able to yield accurate predictions of the amplitude functions with larger time steps than the approximations on which MITIM-E' $(1,1)$ and MOVER-II were based.

The second observation concerns MOVER-I. For both values of epsilon, accurate results were obtained. However, as noted in Table III-2, a substantial number of time steps were required early in the transient. Since MOVER-II was able to produce adequate results with a much smaller number of time steps, it would seem that, at least in this case, the self-selection of time steps is an expensive alternate to using one's knowledge about the transient to select, a priori, the size of $\Delta t$ 。 
As a check on the accuracy of the time synthesis approximation and the multimode approach, Table III-3 compares the magnitude of the flux predicted by SPATKIN at $t=.1 \mathrm{sec}$ at various mesh points across the reactor to the magnitude of the flux predicted at $t=.1 \mathrm{sec}$ by recombining the amplitude functions .0378 and .3379 with their respective trial functions. Table III- 2 and Table III-3 show that these are the amplitude functions predicted at $t=.1$ sec by MOVER-I and, for a sufficiently small time step, by MOVER-II, MITIM-E $(2,0)$ and by $\operatorname{MITIM-E'}(1,1)$ as well.

It appears that the multimode kinetics approximation adequately describes the transient for this case.

\section{Case 2: A Super Prompt Critical Ramp Insertion of Reactivity}

The second transient considered resulted from the ramp insertion of well over a dollar of positive reactivity in the course of a second. For this perturbation, $\Sigma_{a}$ in Region III changed from $0.1949 \iota 2 \mathrm{~cm}^{-1}$ to $.0185001 \mathrm{~cm}^{-1}$ in one second.

Figure III-2 shows the two trial and weight functions selected to bracket the transient. The amplitude functions, $T_{1}(t)$ and $T_{2}(t)$ are then found at various times in Table III-4, where the predictions of MITIM$\mathrm{E}(2,0), \operatorname{MITIM}-\mathrm{E}^{\prime}(1,1)$ and of MOVER-II are presented at various times during the ramp for various choices of $\Delta t$, the size of the time step.

The results presented in Table III-4 indicate first that the $\mathrm{E}_{1,1}^{\prime}(\Delta \mathrm{t}[\mathrm{A}])$ approximation is more capable of handling this ramp reactivity insertion than the $\mathrm{E}_{2,0}(\Delta \mathrm{t}[\mathrm{A}])$ approximation. In fact, the results from MITIM-E(2,0) indicate that the virtue of the $E_{2,0}(\Delta t[A])$ 


\begin{tabular}{|c|c|c|c|c|c|c|c|}
\hline \multirow[b]{3}{*}{$\begin{array}{l}\text { Time } \\
(\mathrm{sec})\end{array}$} & \multicolumn{5}{|c|}{$\begin{array}{c}\text { Table III }-3 \\
\text { Comparison with } \theta-\text { Method }\end{array}$} & & \\
\hline & \multirow[b]{2}{*}{ Method } & \multicolumn{2}{|c|}{ Mesh Point \# 24} & \multicolumn{2}{|c|}{ Mesh Point \#32 } & \multicolumn{2}{|c|}{ Mesh Point \#41 } \\
\hline & & Magnitude & $(\% \text { error })^{*}$ & Magnitude & (\% error) & Magnitude & (\% error) \\
\hline \multirow{3}{*}{0.0} & $\mathrm{a}$ & 1.160 & $(0.0)$ & 1.137 & $(0.0)$ & 1.163 & $(0.0)$ \\
\hline & & & & & & & \\
\hline & $\mathrm{b}$ & 1.160 & $(0.0)$ & 1.157 & $(0.0)$ & 1.163 & $(0.0)$ \\
\hline \multirow{3}{*}{0.1} & $\mathrm{a}$ & .399 & $(.99)$ & .331 & $(.915)$ & .433 & $(.46)$ \\
\hline & & & & & & & \\
\hline & $\mathrm{b}$ & .403 & $(0.0)$ & .328 & $(0.0)$ & .435 & $(0.0)$ \\
\hline
\end{tabular}
* error $\equiv \frac{\text { SPATKIN-MODAL }}{\text { SPATKIN }}$
$\mathrm{a}=$ multimode kinetics
$\mathrm{b}=\theta$-method 
Normalized Trial Function $\left(\psi_{k}(x)\right)$ and Weight Function $\left(\omega_{p}(x)\right)$

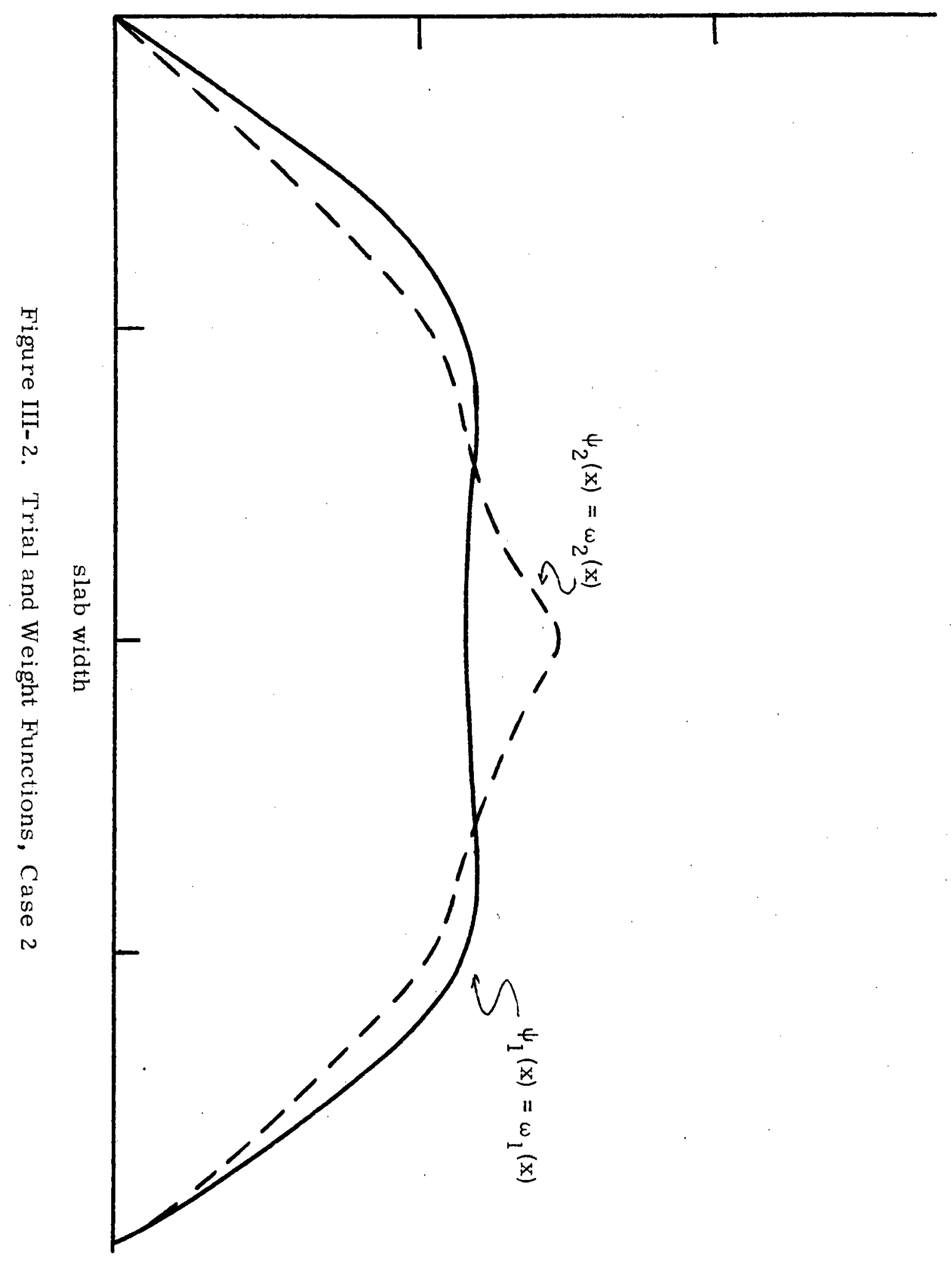




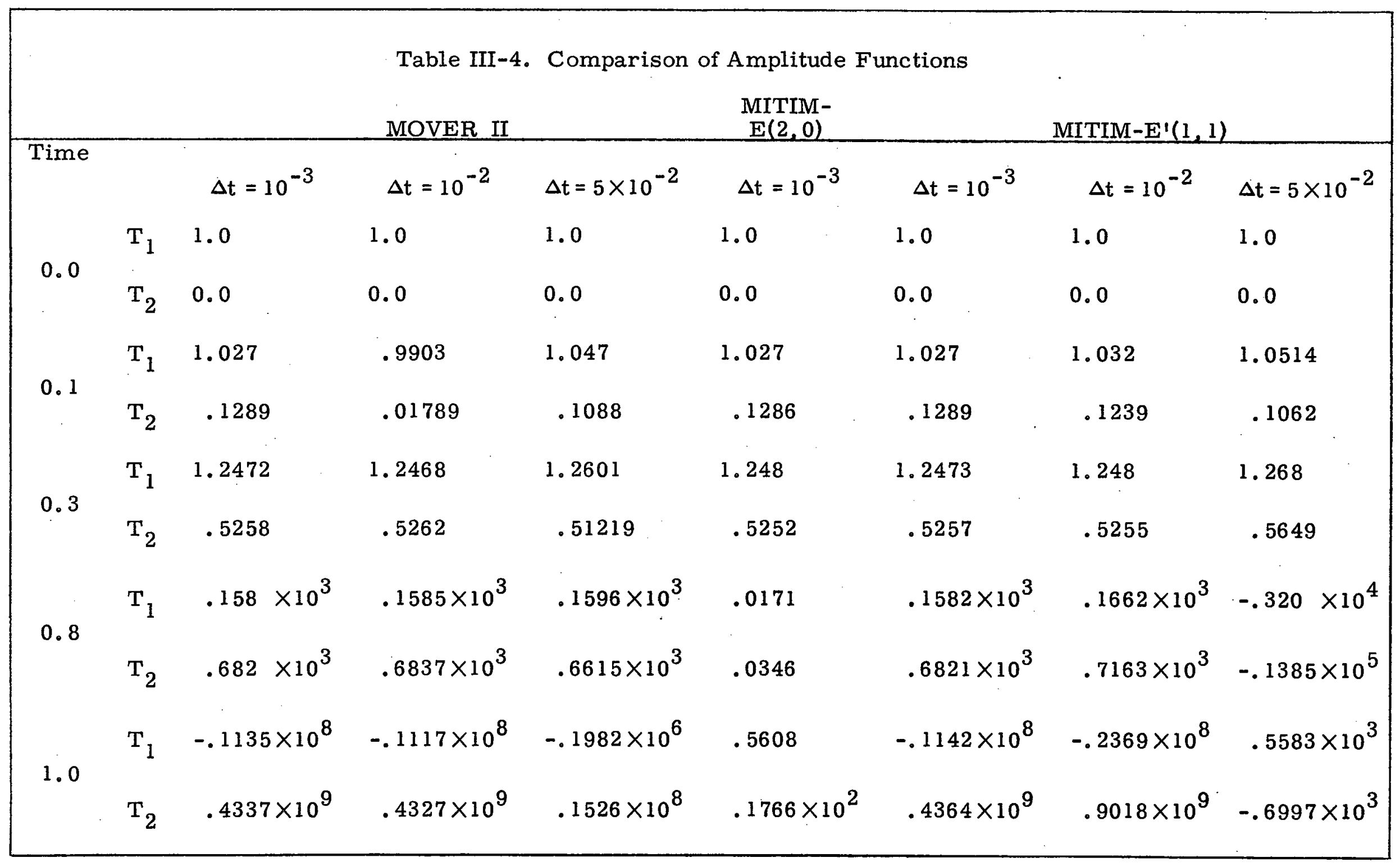


approximation, namely, its ability to reduce properly the influence on the solution of large negative eigenvalues of $[A(t)]$, becomes a vice when the reactor goes supercritical, since then it damps the physically important effect of the large positive eigenvalue equally well.

Table III-4 also seems to indicate that MOVER-II is a little better than MITIM-E' $(1,1)$ in handling this transient. Before reaching this conclusion, however, it is important to note that a comparison of running times on the IBM 370 model 155 reveals that for this problem MITIM-E' $(1,1)$ takes less than half as long per time step as MOVER-II. Although this is clearly not a conclusive argument, it does indicate that if one is concerned with comparing accuracy for the same amount of computational effort, the two approaches are roughly equal in their ability to handle this particular transient.

Again, the results were compared to those of SPATKIN as a check on the accuracy of the time synthesis approximation and the multimode approach. Table III- 5 compares the magnitude of the flux predicted by SPATKIN at $t=.3$ and $1.0 \mathrm{sec}$ at various mesh points across the slab to the magnitudes found by using $T_{1}(.3)=1.247$ and $T_{2}(.3)=.526$ at $t=0.3$ seconds, and by using $T_{1}(1.0)=.114 \times 10^{8}$ and $T_{2}(1.0)=.436 \times$ $10^{9}$ at $t=1.0$ seconds. As in the first problem considered, adequate accuracy was obtained with the multimode approximation.

\section{Case 3: A Step Insertion of Reactivity Less than Prompt Critical}

The last transient considered for this slab reactor was initiated by the step insertion of about $2 / 3$ of a dollar of positive reactivity. This perturbation was produced by changing $\Sigma_{a}$ in Region III abruptly from 


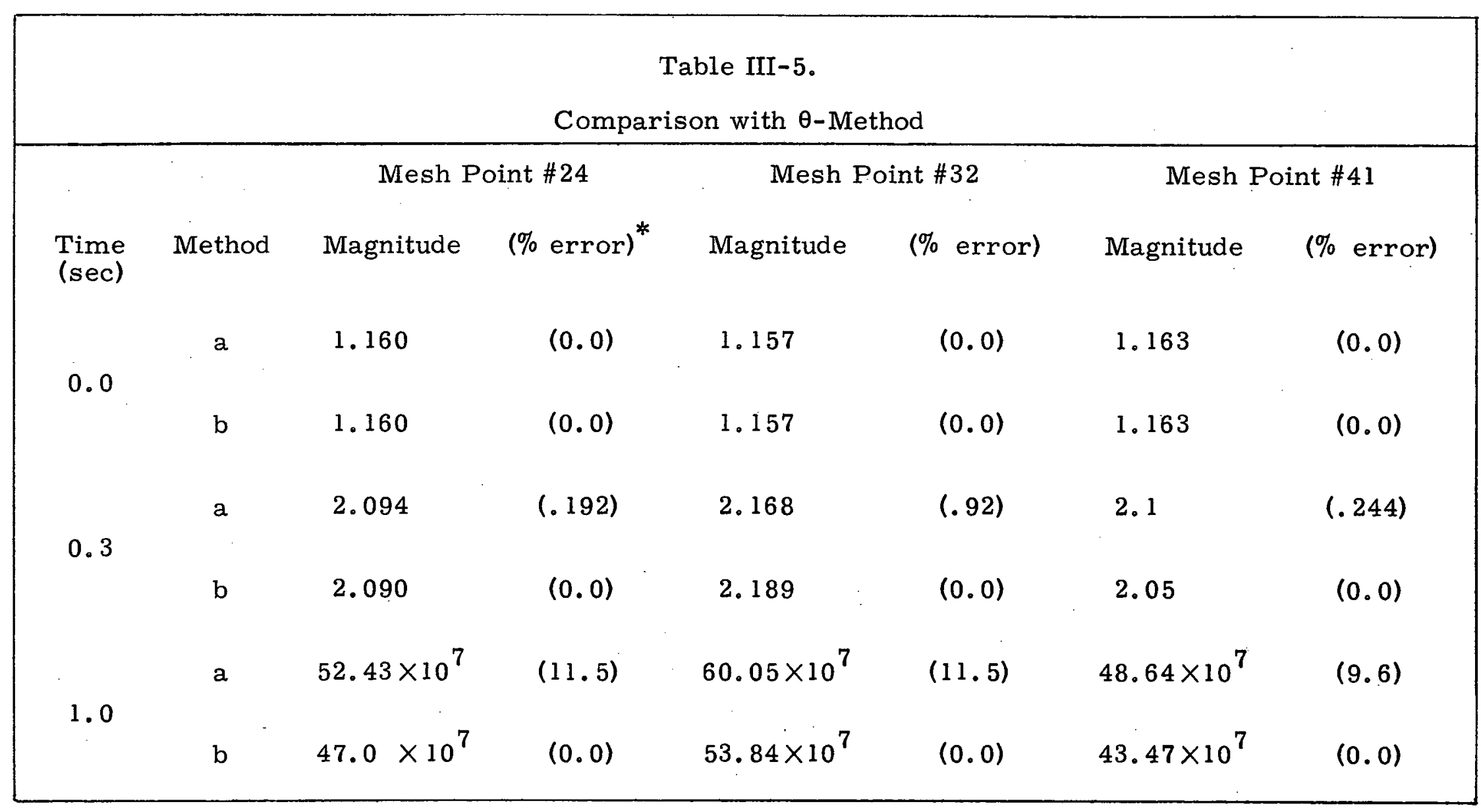

* $\%$ error = SPATKIN-MODAL SPATKIN 
0.194962 to 0.190472 at $t=0^{+}$.

Figure III-3 shows the two trial and weight functions used in equation (3.1). The amplitude functions $T_{1}(t)$ and $T_{2}(t)$ are given in Table III -6 at various times from $t=0$ to $t=1.0$ seconds. As in the preceding example, Table III-6 presents the predictions from MITIM$E(2,0), \operatorname{MITIM}-E^{\prime}(1,1)$ and MOVER-II for different choices of $\Delta t$. These results clearly show the superiority of $E_{2,0}(\Delta t[A])$ approximation in handling this step reactivity insertion. Even with $\Delta t=.5$ seconds, MITIM-E $(2,0)$ provided excellent results.

Finally, the SPATKIN results are presented at $t=.5$ and $t=1.0$ in Table III-7, along with the results produced from the amplitude functions $T_{1}(.5)=-.174$ and $T_{2}(.5)=3.796$ at $t=.5$ seconds and $T_{1}(1.0)=$ -.1999 and $\mathrm{T}_{2}(1.0)=4.173$ at $\mathrm{t}=1.0$ seconds. As in the preceding examples, the multimode approximation produced sufficiently accurate results with two trial functions.

\subsection{Reactor Number Two - Two-Group Results}

The second slab reactor studied here was another $240-\mathrm{cm}$ slab with the critical parameters given in Table III-8. The size of the mesh spacing was $2.5 \mathrm{~cm}$.

This reactor consists of six precursor groups. The relevant information about these groups is given in Table III-9.

The transient studied for this reactor was a positive ramp, induced by linearly decreasing $\Sigma_{a}^{2}$ in Region I by $1 \%$ in 1 second. Figures III-4 and III-5 show the trial and weight functions used to synthesize the flux via equation (3.1). 
Normalized Trial Function $\left(\psi_{2}(x)\right)$ and Weight Function $\left(\omega_{p}(x)\right)$

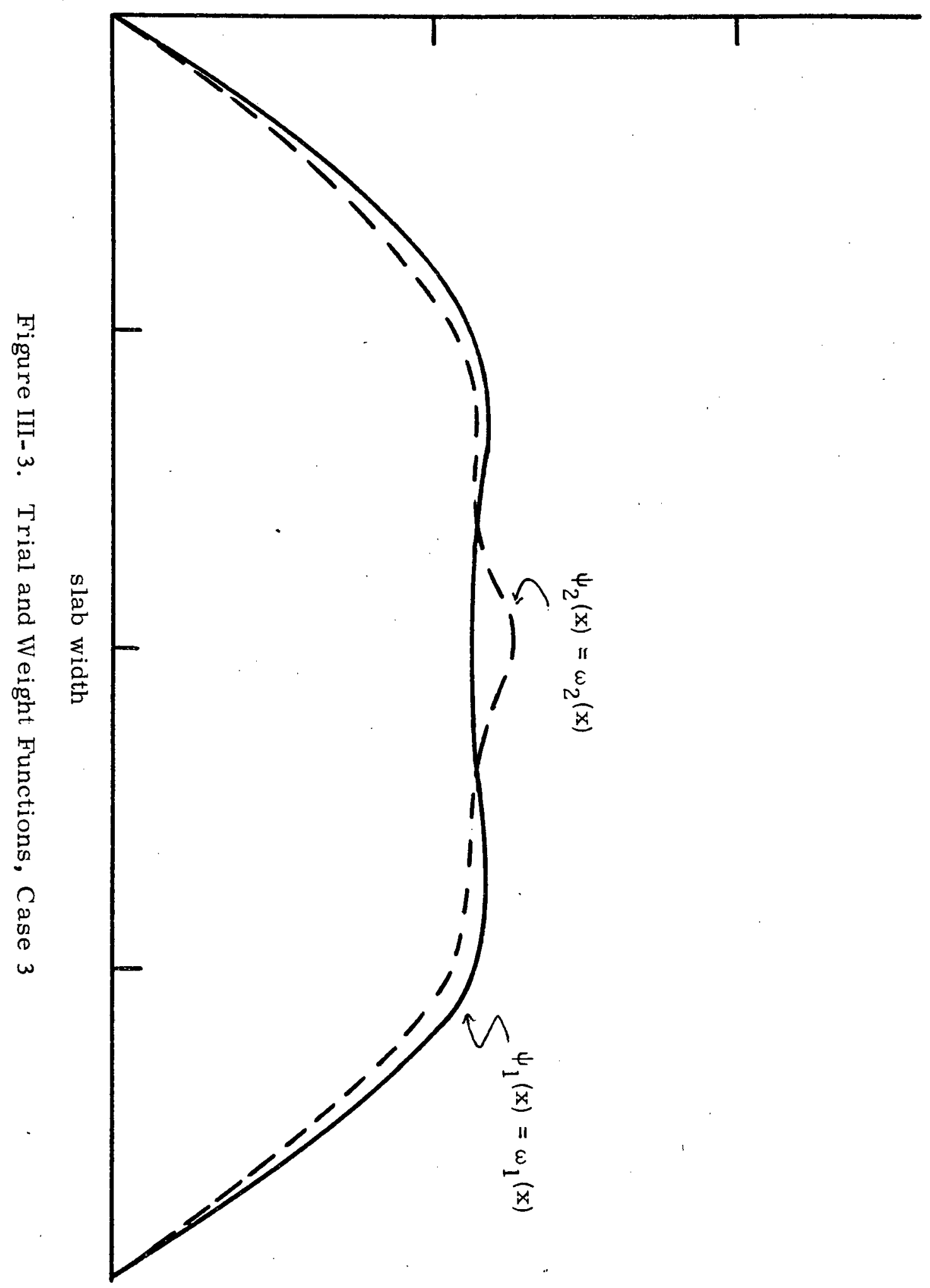


Table III-6. Comparison of Amplitude Functions

\begin{tabular}{|c|c|c|c|c|c|c|c|c|c|c|c|c|}
\hline & & \multicolumn{4}{|c|}{ MOVER II } & \multicolumn{4}{|c|}{ MITIM-E $(2,0)$} & \multicolumn{3}{|c|}{$\operatorname{MITIM-E}(1,1)$} \\
\hline Time & & $\Delta t=10^{-3}$ & $\Delta t=10^{-2}$ & $\Delta t=10^{-1}$ & $\Delta t=.25$ & $\Delta t=10^{-2}$ & $\Delta t=10^{-1}$ & $\Delta t=.25$ & $\Delta t=.5$ & $\Delta t=10^{-2}$ & $\Delta t=10^{-1}$ & $\Delta t=.25$ \\
\hline & $\mathrm{T}_{1}$ & 1.0 & 1.0 & 1.0 & 1.0 & 1.0 & 1.0 & 1.0 & 1.0 & 1.0 & 1.0 & 1.0 \\
\hline & $\mathrm{T}_{2}$ & 0.0 & 0.0 & 0.0 & 0.0 & 0.0 & 0.0 & 0.0 & 0.0 & 0.0 & 0.0 & 0.0 \\
\hline & $\mathrm{T}_{1}$ & -.1521 & -.1335 & .8082 & - & -.1535 & -.1447 & - & 一 & .0217 & -1.191 & - \\
\hline & $\mathrm{T}_{2}$ & 3.450 & 3.438 & 2.387 & - & 3.458 & 3.312 & - & - & 3.289 & 5.254 & - \\
\hline & $\mathrm{T}_{1}$ & -.1738 & -.1601 & .6055 & .8006 & -.1735 & -.1754 & -.1753 & -.1746 & -.1752 & -1.098 & .8786 \\
\hline & $\mathrm{T}_{2}$ & 3.794 & 3.777 & 3.006 & 2.639 & 3.796 & 3.796 & 3.795 & 3.783 & 3.796 & 4.723 & 1.773 \\
\hline & $\mathrm{T}_{\mathrm{I}}$ & -.1984 & -.1848 & .4140 & .7267 & -.1999 & -.1999 & -.1999 & -.1999 & -.1999 & .6453 & .8045 \\
\hline & $\mathrm{T}_{2}$ & 4.171 & 4.154 & 4.140 & 3.217 & 4.173 & 4.173 & 4.172 & 4.171 & 4.173 & 3.329 & 2.761 \\
\hline
\end{tabular}




\begin{tabular}{|c|c|c|c|c|c|c|c|}
\hline & & & Compari & \multicolumn{3}{|c|}{ Comparison with $\theta-$ Method } & \\
\hline \multirow[b]{2}{*}{$\begin{array}{l}\text { Time } \\
\text { (sec) }\end{array}$} & \multirow[b]{2}{*}{ Method } & \multicolumn{2}{|c|}{ Mesh Point \#24 } & \multicolumn{2}{|c|}{ Mesh Point \#32 } & \multicolumn{2}{|c|}{ Mesh Point \#41 } \\
\hline & & Magnitude & $(\% \text { error })^{*}$ & Magnitude & (\% error) & Magnitude & (\% error) \\
\hline & $a$ & 1.160 & $(0.0)$ & 1.157 & $(0.0)$ & 1.163 & $(0.0)$ \\
\hline \multicolumn{8}{|l|}{0.0} \\
\hline & $\mathrm{b}$ & 1.160 & $(0.0)$ & 1.157 & $(0.0)$ & 1.163 & $(0.0)$ \\
\hline & $\mathrm{a}$ & 4.33 & $(.697)$ & 4.62 & $(.435)$ & 4.19 & $(.722)$ \\
\hline \multicolumn{8}{|l|}{0.5} \\
\hline & $\mathrm{b}$ & 4.30 & $(0.0)$ & 4.60 & & 4.16 & \\
\hline & $a$ & 4.75 & $(.422)$ & 5.07 & $(.59)$ & 4.61 & $(.217)$ \\
\hline \multicolumn{8}{|l|}{1.0} \\
\hline & $\mathrm{b}$ & 4.73 & $(0.0)$ & 5.04 & $(0.0)$ & 4.62 & $(0.0)$ \\
\hline
\end{tabular}




\begin{tabular}{|c|c|c|c|}
\hline \multicolumn{4}{|c|}{$\begin{array}{c}\text { Table III-8. } \\
\text { Parameters for Criticality }\end{array}$} \\
\hline $\begin{array}{l}\text { Parameter } \\
\text { (units) }\end{array}$ & $\begin{array}{c}\text { Region I } \\
(0-40 \mathrm{~cm})\end{array}$ & $\begin{array}{c}\text { Region II } \\
(40-200 \mathrm{~cm})\end{array}$ & $\begin{array}{l}\text { Region III } \\
(200-240 \mathrm{~cm})\end{array}$ \\
\hline$D^{l}(\mathrm{~cm})$ & 1.5 & .1 .0 & 1.5 \\
\hline $\mathrm{D}^{2}(\mathrm{~cm})$ & 0.5 & 0.5 & 0.5 \\
\hline$v \Sigma_{\mathrm{f}}^{1}\left(\mathrm{~cm}^{-1}\right)$ & .01677548 & .0083774 & .01677548 \\
\hline$v \Sigma_{a}^{2}\left(\mathrm{~cm}^{-1}\right)$ & .3355096 & .166077252 & .3355096 \\
\hline$\Sigma_{a}^{1}\left(\mathrm{~cm}^{-1}\right)$ & .026 & .020 & .026 \\
\hline$\Sigma_{\mathrm{a}}^{2}\left(\mathrm{~cm}^{-1}\right)$ & .18 & .08 & .18 \\
\hline$\Sigma_{s_{2-1}}\left(\mathrm{~cm}^{-1}\right)$ & .015 & .01 & .015 \\
\hline$x_{\rho}^{1}$ & 1.0 & 1.0 & 1.0 \\
\hline$x_{\rho}^{2}$ & 0.0 & 0.0 & 0.0 \\
\hline $\mathrm{v}_{1}(\mathrm{~cm} / \mathrm{sec})$ & $1.0 \times 10^{7}$ & $1.0 \times 10^{7}$ & $1.0 \times 10^{7}$ \\
\hline $\mathrm{v}_{2}(\mathrm{~cm} / \mathrm{sec})$ & $3.0 \times 10^{5}$ & $3.0 \times 10^{5}$ & $3.0 \times 10^{5}$ \\
\hline
\end{tabular}

In the above table, $\Sigma_{a}^{g}$ is the sum of the macroscopic fission and capture cross sections. 
Table III-9.

Delayed Neutron Parameters

\begin{tabular}{|c|c|c|c|}
\hline ela & $\begin{array}{l}\text { Fractional Yield } \\
\text { into }\end{array}$ & $\begin{array}{l}\text { Fractional Yield } \\
\text { into } \\
\text { Neutron Group } 2\end{array}$ & $\begin{array}{c}\text { Decay } \\
\text { Constant }\left(\sec ^{-1}\right)\end{array}$ \\
\hline
\end{tabular}

1

$2.5 \times 10^{-4}$

0.0

$1.24 \times 10^{-2}$

2

$1.69 \times 10^{-3}$

0.0

$3.05 \times 10^{-2}$

3

$1.47 \times 10^{-3}$

0.0

$1.11 \times 10^{-1}$

4

$2.96 \times 10^{-3}$

0.0

$3.01 \times 10^{-1}$

5

$8.60 \times 10^{-4}$

0.0

1.14

6

$3.20 \times 10^{-4}$

0.0

3.01 


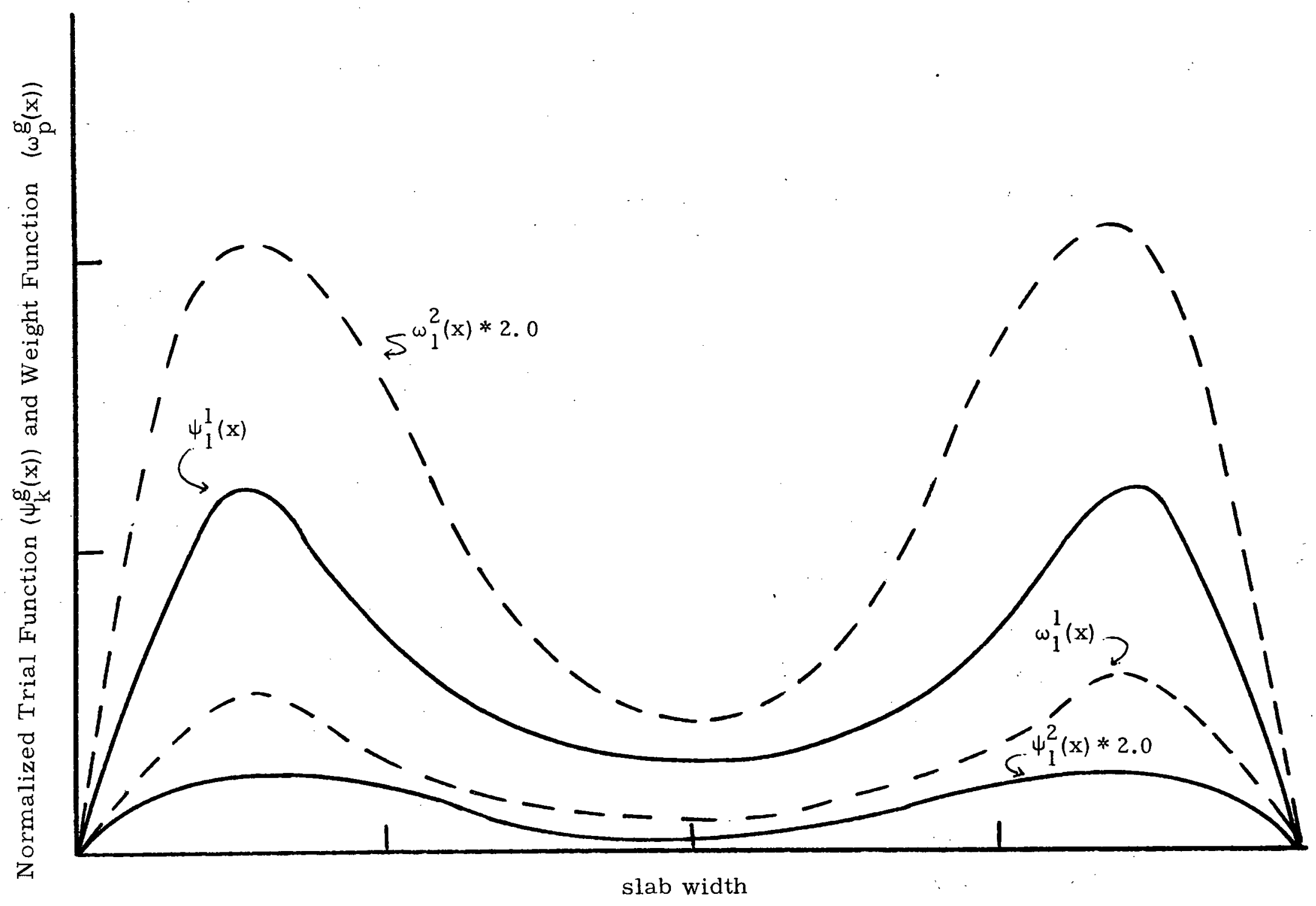

Figure III-4. Trial and Weight Function \#1, Two-Group Problem 


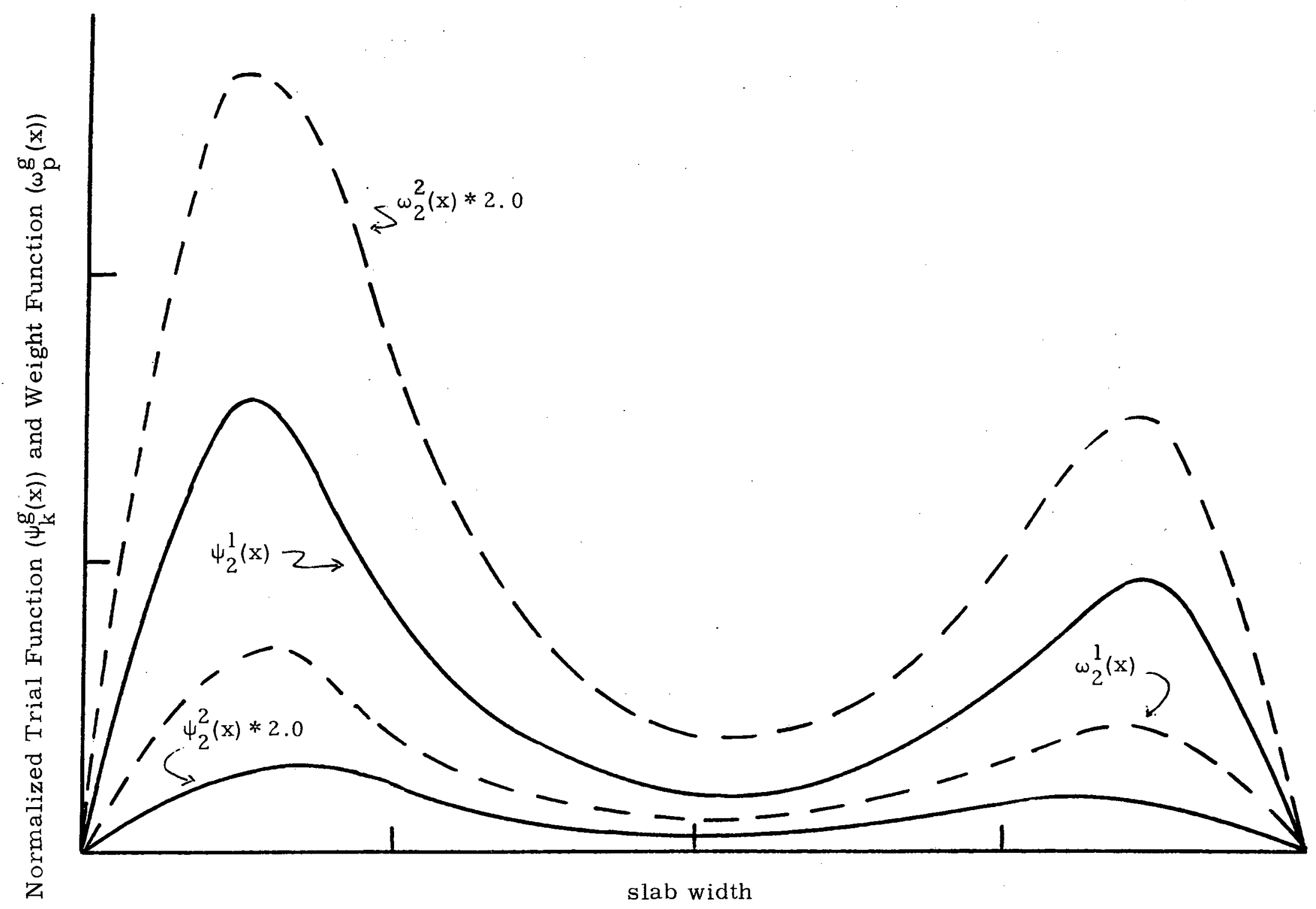

Figure III-5. Trial and Weight Function \#2, Two-Group Problem 
Table III-10 then shows the predictions of $T_{1}(t)$ and $T_{2}(t)$ made by MITIM-E $(2,0)$ and by MITIM-E' $(1,1)$ during the course of the transient for different values of $\Delta t_{\text {. }}$ Again, the $E_{1,1}^{\prime}(\Delta t[A])$ approximation proved to be better than the $\left.E_{2,0}(\Delta t \mid A]\right)$ approximation in handling ramp insertions.

The flux shapes found by recombining the trial and amplitude functions are then compared in Table III-11 to those predicted by GAKIN. These comparisons are made at $t=.5$ and $t=1.0$ seconds at various mesh points with the amplitude functions $\mathrm{T}_{1}(.5)=0.342$ and $\mathrm{T}_{2}(.5)=$ .813 at $t=.5$ seconds and $T_{1}(1.0)=-.1391$ and $T_{2}(1.0)=3.187$ at $t=$ 1.0 seconds. A comparison of these results indicates that the multimode approximation, fully collapsed with only two trial functions, yielded quite satisfactory results.

In this chapter, the results of four numerical studies with two slab reactors have been presented. The next chapter contains the important conclusions to be drawn from these results and ends with some recommendations for future work. 


\begin{tabular}{|c|c|c|c|c|c|c|c|c|c|}
\hline \multicolumn{10}{|c|}{ Table III-10. Comparison of Amplitude Functions } \\
\hline & & \multicolumn{4}{|c|}{ MITIM-E $(2,0)$} & \multicolumn{4}{|c|}{$\operatorname{MITIM}-E^{\prime}(1,1)$} \\
\hline Time & & $\Delta \mathrm{t}=.0025$ & $\Delta t=10^{-2}$ & $\Delta t=10^{-1}$ & $\Delta t=.5$ & $\Delta t=.0025$ & $\Delta t=10^{-2}$ & $\Delta t=10^{-1}$ & $\Delta t=.5$ \\
\hline \multirow{2}{*}{0.0} & $\mathrm{~T}_{1}$ & 1.0 & 1.0 & 1.0 & 1.0 & 1.0 & 1.0 & 1.0 & 1.0 \\
\hline & $\mathrm{T}_{2}$ & 0.0 & 0.0 & 0.0 & 0.0 & 0.0 & 0.0 & 0.0 & 0.0 \\
\hline \multirow{2}{*}{0.2} & $\mathrm{~T}_{1}$ & .7933 & .8083 & .8447 & & .7927 & .7927 & .7968 & \\
\hline & $\mathrm{T}_{2}$ & .2722 & .2523 & .2043 & & .2729 & .2729 & .2693 & \\
\hline \multirow{2}{*}{0.5} & $\mathrm{~T}_{1}$ & .3428 & .3479 & .4212 & .7042 & .3421 & .3421 & .3386 & .3328 \\
\hline & $\mathrm{T}_{2}$ & .8724 & .8659 & .7961 & .3937 & .8734 & .8734 & .8766 & .8840 \\
\hline \multirow{2}{*}{0.8} & $\mathrm{~T}_{1}$ & -.4482 & -.4401 & -.3134 & & -.4493 & -.4493 & -.4473 & \\
\hline & $\mathrm{T}_{2}$ & 1.929 & 1.918 & 1.751 & & 1.930 & 1.930 & 1.929 & \\
\hline \multirow{2}{*}{1.0} & $\mathrm{~T}_{1}$ & -1.374 & -1.3773 & -1.1743 & -.4292 & -1.391 & -1.391 & -1.391 & -1.437 \\
\hline & $\mathrm{T}_{2}$ & $3.064^{*}$ & 3.170 & 2.902 & 1.913 & 3.187 & 3.187 & 3.188 & 3.254 \\
\hline
\end{tabular}

${ }^{*}$ Here, MITIM-E $(2,0)$ apparently predicted $\mathrm{T}_{2}$ with more accuracy using a $\Delta t$ of $10^{-2}$ than it did using $\Delta t=.0025$. This is thought to be due to the fact that differences are being taken between small numbers which are being multiplied by $\Delta t$. Thus, if $\Delta$ t gets too small, roundoff error becomes a problem. 


\begin{tabular}{|c|c|c|c|c|c|c|c|c|c|c|}
\hline & \multicolumn{10}{|c|}{ Table III-11. Comparison with GAKIN Method } \\
\hline & & & \multicolumn{2}{|c|}{ Mesh Point \# 8} & \multicolumn{2}{|c|}{ Mesh Point \#40 } & \multicolumn{2}{|c|}{ Mesh Point \# 56} & \multicolumn{2}{|c|}{ Mesh Point \#91 } \\
\hline \multirow{7}{*}{ 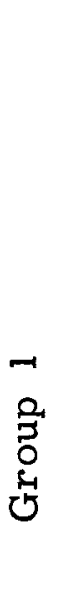 } & $\begin{array}{l}\text { Time } \\
(\mathrm{sec})\end{array}$ & Method & Magnitud & $(\% \text { error })^{*}$ & Magnitude & (\% error) & Magnitude & (\% error) & Magnitude & (\% error) \\
\hline & \multirow{2}{*}{0.0} & a & 1.8368 & $(0.0)$ & . 5760 & $(0.0)$ & .5379 & $(0.0)$ & 1.628 & $(0.0)$ \\
\hline & & $\mathrm{b}$ & 1.8368 & $(0.0)$ & .5760 & $(0.0)$ & .5379 & $(0.0)$ & 1.628 & $(0.0)$ \\
\hline & \multirow{2}{*}{0.5} & a & 2.597 & $(4.17)$ & .7121 & $(1.34)$ & .5846 & $(1.62)$ & 1.6684 & $(2.17)$ \\
\hline & & $b$ & 2.493 & $(0.0)$ & .7027 & $(0.0)$ & .5753 & $(0.0)$ & 1.6329 & $(0.0)$ \\
\hline & \multirow{2}{*}{1.0} & $a$ & 4.629 & $(3.68)$ & 1.091 & $(7.0)$ & .7139 & $(2.3)$ & 1.7919 & $(.35)$ \\
\hline & & $b$ & 4.805 & $(0.0)$ & 1.1732 & $(0.0)$ & .7287 & $(0.0)$ & 1.7314 & $(0.0)$ \\
\hline \multirow{6}{*}{ 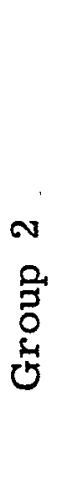 } & \multirow{2}{*}{0.0} & a & .1521 & $(0.0)$ & .07234 & $(0.0)$ & .06755 & $(0.0)$ & .1348 & $(0.0)$ \\
\hline & & $\mathrm{b}$ & .1521 & $(0.0)$ & .07234 & $(0.0)$ & .06755 & $(0.0)$ & .1348 & $(0.0)$ \\
\hline & \multirow{2}{*}{0.5} & $\mathrm{a}$ & .2166 & $(4.5)$ & .08947 & $(1.4)$ & .0734 & $(1.52)$ & .1381 & $(2.14)$ \\
\hline & & $\mathrm{b}$ & .2072 & $(0.0)$ & .0882 & $(0.0)$ & .0723 & $(0.0)$ & .1352 & $(0.0)$ \\
\hline & \multirow{2}{*}{1.0} & $\mathrm{a}$ & .3891 & $(3.1)$ & .1356 & $(7.9)$ & .0897 & $(1.97)$ & .14833 & $(3.43)$ \\
\hline & & $b$ & .4016 & $(0.0)$ & .1473 & $(0.0)$ & .0915 & $(0.0)$ & .1434 & $(0.0)$ \\
\hline
\end{tabular}

$*$ error $\equiv \frac{\text { GAKIN-MODAL }}{\text { GAKIN }}$

$\mathrm{a}=$ multimode kinetics

$\mathrm{b}=$ GAKIN method 


\section{CHAPTER IV \\ CONCLUSIONS AND RECOMMENDATIONS}

In Chapter III, numerical results were presented for four problems which were designed to test the efficiency of the $E_{2,0}(\Delta t[A])$ and $\mathrm{E}_{1,1}^{\prime}(\Delta \mathrm{t}[\mathrm{A}])$ approximations described in Chapter II. In the following section of the present chapter an attempt is made to present a few conclusions gleaned from the numerical results of the previous chapter. Section 4.2 then recommends several topics worthy of future consideration.

\subsection{Conclusions}

The first conclusion that one should make from the results of Chapter III is that the $E_{2,0}(\Delta t[A])$ and $E_{1,1}^{\prime}(\Delta t[A])$ approximations behaved in such a manner as to justify the theoretical motivation behind their selection. For the step reactivity insertions, the $\mathrm{E}_{2,0}(\Delta \mathrm{t}[\mathrm{A}])$ approximation proved more suited than the $E_{1,1}^{\prime}(\Delta t[A])$ in effectively damping out the influence of those large negative eigenvalues of [A]. (Unfortunately, this same characteristic made the approximation grossly underestimate the growth of a prompt-critical reactor.) Also, as expected, the $E_{1,1}^{1}(\Delta t[A])$ approximation proved superior in describing rampinduced transients.

In addition, one may state at least two other conclusions regarding the successful generalization of da Nobrega's work in point kinetics 34 to attack the multimode kinetics equations considered by Fuller, Meneley, and Hetrick. ${ }^{39}$ First, it should be noted that in all the problems 
considered adequate agreement was obtained between the predictions of the multimodal approximations and an independent method. More than this, however, the $E_{2,0}(\Delta t[A])$ and $E_{1,1}^{\prime}(\Delta t[A])$ approximations seemed to be at least roughly equal to, and usually better than, the temporal integration approach taken by Fuller, Meneley, and Hetrick. ${ }^{39}$ Only in the second transient considered did the temporal integration approach appear at first glance to be somewhat better than the other two approaches. In more complicated models, however, the efficiency of the $E_{2,0}(\Delta t[A])$ and $E_{1,1}^{\prime}(\Delta t[A])$ approximations vis a vis the approach taken by Fuller, Meneley, and Hetrick ${ }^{39}$ may increase since an increase in the number of precursors considered will necessitate an increase in the number of integrals and exponentials taken in the temporal integration procedure.

Secondly, the success of the new approach investigated stemmed not only from the characteristics of the Padé approximations employed, but in large part from the manner in which the $\mathrm{E}_{2,0}(\Delta \mathrm{t}[\mathrm{A}])$ and $E_{1,1}^{\prime}(\Delta t[A])$ approximations were applied to the solution of the multimode kinetics equations. As a consequence of the way in which this was done, one need only invert a relatively small matrix (at most of order $(G \times K)^{2}$, where $G \equiv$ number of groups and $K \equiv$ number of trial functions) to step out from $t_{p}$ to $t_{p+1}$. Thus the application produced, in each case, an advancement matrix with the capability of generating quite cheaply the vector $\left[\psi_{\mathrm{p}+1}\right]$ in terms of the vector $\left[\psi_{\mathrm{p}}\right]$.

\section{2 Recommendations for Future Work}

The present work suggests that there are at least two major areas which require additional consideration. The first is concerned with 
the time synthesis approximation itself; the second is concerned with the use of the $E_{p, q}(\Delta t[A])$ approximations of Chapter II to approximate accurately the exponential, $\exp (\Delta t[A])$ of equation (1.29).

The first recommendation for future work stems from uncertainties with the time synthesis approximation. At present, not much has been published to indicate that there exists a very precise idea of the "nearness" of the solution space of the synthesized equations to that of the multigroup diffusion equations. As a result, one is not able to put adequate error bounds on the solutions of the synthesized equations. Thus there is a constant danger of encountering cases for which the synthesis approximation gives unexpectedly poor results. It appears, then, that a theoretically clearer notion is needed of how to select trial functions such that the resulting solution space contains vectors which accurately approximate the solutions of the multigroup neutron diffusion equations.

The second recommendation for future work is concerned with the capacity of the Pade matrix approximations to approximate accurately $\exp (\Delta t[A])$. In this regard, it would be valuable to extend the work of Porsching 47 in order to quantify the eigenvalue spectrum of the general multimode kinetics matrix $[A(t)]$. If this were done, a precise theoretical foundation could be established for specifying the accuracy of the Padé matrix approximations. 


\section{REFERENCES}

1. S. Kaplan, "Synthesis Methods in Reactor Analysis," Adv. in Nucl. Sci. and Tech., 3, 234 (1966).

2. A. F. Henry, "Space-Time Reactor Kinetics," 22. 243 Course Notes, Massachusetts Institute of Technology (1969), p. 26 (unpublished).

3. Donald R. Ferguson, "Solution of the Space-Dependent Reactor Kinetics Equations in Three Dimensions," Ph. D. Thesis, Department of Nuclear Engineering, Massachusetts Institute of Technology, MIT-NE- 132 (August, 1971), p. 15.

4. J. Barclay Andrews II and K. F. Hansen, "Numerical Solution of the Time-Dependent Multigroup Diffusion Equations," Nucl. Sci. and Eng., 31, 304 (1968).

5. W. M. Stacy, Jr., Space-Time Nuclear Reactor Kinetics, New York: Academic Press, Inc: (1969), p.52.

6. Donald R. Ferguson, op. cit., p. 28.

7. Ibid., Chapter 3.

8. A. F. Henry, "The Application of Reactor Kinetics to the Analysis of Experiments," Nucl. Sci. and Eng. , 3, 52 (1958).

9. S. Kaplan, A. F. Henry, S. B. Margolis, and J. J. Taylor, "SpaceTime Reactor Dynamics," Geneva Conf. (A/Conf. 28/p/271).

10. A. F Henry, "Space-Time Reactor Kinetics," 22.243 Course Notes, Massachusetts Institute of Technology (1969), p. 82 (unpublished).

11. A. F. Henry, op. cit., p. 93.

12. H. L. Garabedian and C. B. Leffert, "A Time-Dependent Analysis of Spatial Flux Distributions," Nucl. Sci. and Eng., 6, 26 (1959).

13. A. F. Henry, op. cit., p. 110 .

14. Ibid.

15. S. Kaplan, O. J. Marlowe, and J. Bewick, "Application of Synthesis Techniques to Problems Involving Time Dependence," Nucl. Sci. and Eng., 18, 163 (1964).

16. R. Avery, "Theory of Coupled Reactors," Proc. 2nd U.N. Conf. on Peaceful Uses of A tomic Energy, Geneva, 12, 182 (1958), Paper No. 1858.

17. J. B. Yasinsky and S. Kaplan, "Synthesis of Three-Dimensional Flux Shapes Using Discontinuous Sets of Trial Functions," Nucl. Sci. and Eng., 28, 426 (1967).

18. E. L. Wachspress, R. D. Burgess, and S. Baron, "Multichannel Flux Synthesis," Nucl. Sci. and Eng. , 12, 381 (1962).

19. E. L. Fuller, D. A. Meneley, and D. L. Hetrick, "Weighted Residual Methods in Space-Dependent Reactor Dynamics," Nucl. Sci. and Eng., 40, 206 (1970). 
20. A. F. Henry, "The Application of Reactor Kinetics to the Analysis of Experiments," Nucl. Sci. and Eng. , 3, 52 (1958).

21. Karl Ott, "Quasistatic Treatment of Spatial Phenomena in Reactor Dynamics," Nucl. Sci. and Eng. , 26, 563 (1966).

22. Wallach, WAPD-B (1950).

23. H. B. Smets, Second Geneva Conference (A/Conf./p/107).

24. J. C. Vigil, "Solution of the Reactor Kinetics Equations by Analytic Continuation," Nucl. Sci. and Eng. , 29, 392 (1967).

25. T. Auerbach, J. Mennig, and G. Sarlos, "The Application of Lie Series to the Time Dependent Reactory Theory," Proc. Brookhaven Conf. on Ind. Needs Acad. Research in Reactor Kinetics, BNL50117 , p. 306, Brookhaven National Laboratory (1968).

26. G. R. Keepin and C.W. Cox, "General Solution of the Reactor Kinetics Equations," Nucl. Sci. and Eng. , 8, 670 (1960),

27. W. R. Rhyne and A. C. Lapsley, "Numerical Solution of the Timeand Space-dependent Multigroup Neutron Diffusion Equations," Nucl. Sci. and Eng., 40, 91 (1970).

28. J. J. Kaganove, "Numerical Solution of the One-Group, SpaceIndependent Reactor Kinetics Equations for Neutron Density Given the Excess Reactivity," ANL-6132, Argonne National Laboratory (1960).

29. E. R. Cohen, "Some Topics in Reactor Kinetics," Proc. 2nd U.N. Intern. Conf. Peaceful Uses At. Energy, 11, 302 (1958).

30. K. F. Hansen, B. V. Koen, and W. W. Little, Jr., "Stable Numerical Solution of the Reactor Kinetics Equations," Nucl. Sci. and Eng. 22, 51 (1965).

31. C. M. Kang, "Piecewise Polynomial Approximations for the Point Kinetics Equations," Trans. Am. Nucl. Soc. , 14, 201 (1971).

32. K. F. Hansen, E. R. Kleban, and I. J. Chang, "A New Method of Integrating the Point Kinetics Equations," Trans. Am. Nucl. Soc., 12, 617 (1969).

33. T. A. Porsching, "Numerical Solution of the Reactor Kinetics Equations by Approximate Exponentials," Nucl. Sci. and Eng., 25, 183 (1965).

34. J. A. W. da Nóbrega, "A New Solution of the Point Kinetics Equations," Nucl. Sci. and Eng. , 46, 366 (1971).

35. M. Izumi and T. Noda, "An Implicit Method for Solving the Lumped Parameter Reactor Kinetics Equations by Repeated Extrapolation," Nucl. Sci. and Eng., 41, 299 (1970).

36. H. D. Brown, "A General Treatment of Flux Transients," Nucl. Sci. and Eng., 2, 687 (1957). 
37. A. F. Henry, A. V. Vota, "WIGL-2 - A Program for the Solution of the One-Dimensional, Two-Group Space-Time Diffusion Equations Accounting for Temperature, Xenon, and Control Feedback," WAPD-TM-532, Bettis A tomic Power Laboratory (1965).

38. R. O. Brittan, "Some Problems in the Safety of Fast Reactors," ANL-5577, Argonne National Laboratory (1956).

39. E. L. Fuller, D. A. Meneley, and D. L. Hetrick, op. cit.

40. E. L. Fuller, "Weighted-Residual Methods in Space-Dependent Reactor Dynamics," ANL-7565, Argonne National Laboratory (1969).

41. H. Padé, "Sur la représentation approcheé d'une fonction par des fractions rationnelles," thése, Ann. de l'Ec. Nor. (3) 9, 93 (1892).

42. Richard S. Varga, Matrix Iterative Analysis, Sec. 8. 3, New Jersey: Prentice-Hall, Inc. (1962).

43. P. M. Hummel and C. L. Seebeck, "A Generalization of Taylor's Theorem," Amer. Math. Monthly, 56, 243 (1949).

44. P. D. Lax and R. D. Richtmyer, "Survey of the Stability of Linear Finite Difference Equations," Comm. Pure Appl. Math. , 9, 267 (1956).

45. R. D. Richtmyer and K. W. Morton, Difference Methods for InitialValue Problems, 2nd Ed., Chap. 3, New York, London, and Sidney: Interscience Publishers (Wiley) (1967).

46. Ibid., Chap. 4.

47. T. A. Porsching, "On the Spectrum of a Matrix Arising from a Problem in Reactor Kinetics," SIAM J..Appl. Math., 16, 301 (1968).

48. J. A. W. da Nobrega, op. cit.

49. J. Crank and P. Nicholson, "A Practical Method for Numerical Evaluation of Solutions of Partial Differential Equations of the Heat-Conduction Type," Proc. Cambridge Philos. Soc. , 43, 50 (1947).

50. William H. Reed, Diffuse (A one-dimensional static program), available from the code library, Department of Nuclear Engineering, Massachusetts Institute of Technology.

51. E. L. Fuller, Wierd (A program to solve the multimode kinetics equations - obtained via personal communication between $T$. Yarman (MIT) and E. L. Fuller).

52. J. A. W. da Nóbrega, Personal communication (August, 1971).

53. K. F. Hansen and S. R. Johnson, "GAKIN - A One-Dimensional Multigroup Kinetics Code," GA-7543, General Atomic Division of General Dynamics (1967). 
APPENDIX I

$$
\underline{\text { Proof of }[e]=\left[a b^{-1} a+b\right]^{-1} \text { and }[z\rfloor=\left\lfloor b^{-1} a e\right]=\left[b a^{-1} b+a\right]^{-1}}
$$

\section{Proof:}

\section{Given that}

$$
[\gamma]^{-1}=[a-b i]^{-1}=\lfloor z+e i]
$$

prove that

$$
[e]=\left[a b^{-1} a+b\right]^{-1}
$$

and that

$$
\lfloor z\rfloor=\left\lfloor b^{-1} a e\right]=\left\lfloor b a^{-1} b+a\right\rfloor^{-1}
$$

If

$$
[a-b i]^{-1}=[z+e i]
$$

then

$$
[\mathrm{a}-\mathrm{b} \mathrm{i}][\mathrm{z}+\mathrm{ei}]=\lfloor\mathrm{I}]
$$

and

$$
[a z+b e+(-b z+a e) i]=[I]
$$

This, in turn, implies that

$$
[\mathrm{az}]=\lfloor\mathrm{I}-\mathrm{be}\rfloor
$$


and that

$$
[\mathrm{bz}]=[\mathrm{ae}] \text {. }
$$

Therefore, one may write

$$
\lfloor z]=\left\lfloor b^{-1} a e\right]
$$

Substituting this expression for $[z]$ into (I. 1) yields:

$$
\left[a b^{-1} a e\right]=[I-b e]
$$

which may be rearranged as

$$
\left[a b^{-1} a+b\right][e]=[I] \text {. }
$$

Thus one may write

$$
[e]=\left[a b^{-1} a+b\right]^{-1}
$$

and, from (I. 3)

$$
[z]=\left[a+b a^{-1} b\right]^{-1}
$$

Q.E.D. 


\section{APPENDIX II}

\section{Proof of Identities}

Given that

$$
\begin{aligned}
& {[z]=\left[b^{-1} a e\right]} \\
& {[e]=\left[b+a b^{-1} a\right]^{-1}} \\
& {[a]=\left[I-\frac{\Delta t}{2}[\Lambda]^{-1}\lfloor\rho]+\sum_{i}^{I} \Delta t P_{i}\left(1+\Delta t \lambda_{i}\right)\left[\mu_{i}\right]\right]} \\
& {[b]=\left[\frac{\Delta t}{2}[\Lambda]^{-1}[\rho]-\sum_{i}^{I} \Delta t P_{i}\left[\mu_{i}\right]\right]}
\end{aligned}
$$

prove that

$$
[z][e]-[e][z]-\sum_{i=1}^{I} \Delta t^{2} P_{i} \lambda_{i}\left[[e]\left[\mu_{i}\right][z]-[z]\left[\mu_{i}\right][e j]=0\right.
$$

and that

$$
[e]^{2}+[z]^{2}+\sum_{i=1}^{I} \Delta t^{2} P_{i} \lambda_{i}\left[[e]\left[\mu_{i}\right][e]+[z]\left[\mu_{i}\right][z]\right]=[e]+[z]
$$

From (II. 3) and (II. 4) one may write

$$
[a]=-[b]+\left[I+\sum_{i}^{I} \Delta t^{2} P_{i} \lambda_{i}\left[\mu_{i}\right]\right] .
$$


Also, since $[a-b]^{-1}=\left[z+e_{i}\right]$

$$
\left[z+e_{i}\right]\left[a-b_{i}\right]=[I]=[z a+e b+(e a-z b) i]
$$

which implies that

$$
[z]=\left[e a b^{-1}\right]
$$

Next, substituting (II. 7) into (II. 1) yields

$$
[z]=-[e]+\left[b^{-1} e\right]+\sum_{i}^{I} \Delta t^{2} P_{i} \lambda_{i}[b]^{-1}\left[\mu_{i}\right][e]
$$

and substituting (II. 7) into (II.8) yields

$$
[z]=-[e]+\left[e b^{-1}\right]+\sum_{i}^{I} \Delta t^{2} P_{i} \lambda_{i}[e]\left[\mu_{i}\right][b]^{-1}
$$

Applying (II. 9) and (II. 10) with I = 2 to equation (II. 5) completes the proof of the first identity, since this yields

$$
\begin{aligned}
& +[e]^{2}-\left[e b^{-1} e\right]-\sum_{i} \Delta t^{2} P_{i} \lambda_{i}\left[e b^{-1} \mu_{i} e\right] \\
& -[e]^{2}+\left[e b^{-1} e\right]+\sum_{i} \Delta t^{2} P_{i} \lambda_{i}\left[e \mu_{i} b^{-1} e\right] \\
& +\sum_{i} \Delta t^{2} P_{i} \lambda_{i}\left[e \mu_{i} e\right]-\sum_{i} \Delta t^{2} P_{i} \lambda_{i}\left[e \mu_{i} b^{-1} e\right] \\
& -\left(\sum_{i} \Delta t^{2} P_{i} \lambda_{i}\right)\left(\sum_{i} \Delta t^{2} P_{i} \lambda_{i}\right)\left[e \mu_{i} b^{-1} \mu_{i} e\right] \\
& -\sum_{i} \Delta t^{2} P_{i} \lambda_{i}\left[e \mu_{i} e\right]+\sum_{i} \Delta t^{2} P_{i} \lambda_{i}\left[e b^{-1} \mu_{i} e\right] \\
& +\left(\sum_{i} \Delta t^{2} P_{i} \lambda_{i}\right)\left(\sum_{i} \Delta t^{2} P_{i} \lambda_{i}\right)\left[e \mu_{i} b^{-1} \mu_{i} e\right]=0 .
\end{aligned}
$$


To prove identity (II.6), one first premultiplies (II.9) by [b]. Thus

$$
\left.-[b][z]-[b][e]+[e]+\sum_{i=1}^{I} \Delta t^{2} P_{i} \lambda_{i} L \mu_{i}\right][e]=0
$$

Substituting (II. 1) yields

$$
[-a-b][e]+[e]+\sum_{i=1}^{I} \Delta t^{2} P_{i} \lambda_{i}\left[\mu_{i}\right][e]=0
$$

Then, premultiplying by $[\mathrm{e}]$, one obtains:

$$
[e][-a-b][e]+[e]^{2}-\sum_{i=1}^{I} \Delta t^{2} P_{i} \lambda_{i}\left[\mu_{i}\right][e]=0
$$

Also, from equation (II. 8), one may write

$$
[e]=[z][b][a]^{-1}
$$

and, from (II. 1)

$$
[\mathrm{e}]=[\mathrm{a}]^{-1}[\mathrm{~b}][\mathrm{z}]
$$

Using (II. 12) and (II. 13) one may show that

$$
[z][a][b]^{-1}=[b]^{-1}[a][z]
$$

Substituting (II. 11) into (II. 9) yields

$$
-[e]-[z]+[b]^{-1}[z][b][a]^{-1}+\sum_{i=1}^{I} \Delta t^{2} P_{i} \lambda_{i}[b]^{-1}\left[\mu_{i}\right\rfloor[z\rfloor[b]\left[a^{-1}\right\rfloor .
$$


Postmultiplying by $[a][b]^{-1}$, premultiplying by [e][a], and using (II. 14) then yields

$$
\left.[z][-a-b][z]+[z]^{2}+\sum_{i=1}^{I} \Delta t^{2} P_{i} \lambda_{i}[z]_{\mu_{i}}\right][z]=0
$$

Adding (II. 15) and (II. 11) then reveals that

$$
\begin{aligned}
& {[z]^{2}+[e]^{2}+\sum_{i=1}^{I} \Delta t^{2} P_{i} \lambda_{i}\left[[z]\left[\mu_{i}\right][z]+[e]\left[\mu_{i}\right][e]\right]} \\
& \quad=[e][a+b][e]+[z][a+b][z] .
\end{aligned}
$$

Substituting (II.13) into the right hand side of (II. 16) and making use of the fact (proven in Appendix I) that $[\mathrm{b}][\mathrm{e}]=[\mathrm{I}-[\mathrm{a}][\mathrm{z}]]$, one may finally show that

$$
[e][a+b][e]+[z][a+b][z]=[e]+[z] .
$$

This completes the proof of the identity given by equation (II. 6). 
APPENDIX III

An Example of How [REAL] May Be Structured

Using the Identities (2.34) and (2.35)

The following example is given to show how one might go about using the identities considered in Appendix II to restructure the matrix [REAL] of equation (2.33). In performing the calculation two precursor groups are considered.

With two groups of delayed neutron emitters [REAL] may be written as

$$
[R E A L]=\left[\begin{array}{lll}
{\left[R_{11}\right]} & {\left[R_{12}\right]} & {\left[R_{13}\right]} \\
{\left[R_{21}\right]} & {\left[R_{22}\right]} & {\left[R_{23}\right]} \\
{\left[R_{31}\right]} & {\left[R_{32}\right]} & {\left[R_{33}\right]}
\end{array}\right]+[L]
$$

where the matrix elements $\left[R_{i j}\right]$ are found by expanding the right-hand side of equation (2.33).

The matrix elements defined by this expansion, however, can be quite complicated. The procedure discloses, for example, that

$$
\begin{aligned}
{\left[R_{11}\right]=} & {[z]^{2}+[e]^{2}+\Delta t^{2} P_{1} \lambda_{1}[z]\left[\mu_{1}\right][z]+\Delta t^{2} P_{1} \lambda_{1}[e]\left[\mu_{1}\right][e] } \\
& +\Delta t^{2} P_{2} \lambda_{2}[z]\left[\mu_{2}\right][z]+\Delta t^{2} P_{2} \lambda_{2}[e]\left[\mu_{2}\right][e]
\end{aligned}
$$

Fortunately, the identities of equations (2.34) and (2.35) may be used to simplify considerably the derived expressions for the matrices $\left[R_{i j}\right]$. Thus $\left[R_{11}\right]$ becomes, via the application of (2.35), equal to [e]+ $[z]$.

In a similar way the remaining matrix elements of [REAL] may be 
found by expanding the right-hand side of (2.33) and then simplified using (2.34) and (2.35).

If this is done, one finds that

$$
\begin{aligned}
& {\left[\mathrm{R}_{11}\right]=[\mathrm{z}]+[\mathrm{e}]} \\
& {\left[\mathrm{R}_{12}\right]=\Delta \mathrm{tP}{ }_{1} \lambda_{1}\left(2+\Delta \mathrm{t} \lambda_{1}\right)[\mathrm{z}]+\Delta \mathrm{t}^{2} \mathrm{P}_{1} \lambda_{1}^{2}[\mathrm{e}]} \\
& {\left[R_{13}\right]=\Delta \mathrm{tP}_{2} \lambda_{2}\left(2+\Delta t \lambda_{2}\right)[\mathrm{z}]+\Delta \mathrm{t}^{2} \mathrm{P}_{2} \lambda_{2}^{2}[\mathrm{e}]} \\
& {\left[R_{21}\right]=\Delta t^{2} P_{1} \lambda_{1}\left[\mu_{1}\right][e]+\Delta t P_{1}\left(2+\Delta t \lambda_{1}\right)\left[\mu_{1}\right][z]} \\
& {\left[R_{22}\right]=\Delta t^{2} P_{1} \lambda_{1}\left(1-4 P_{1}-2 \Delta t P_{1} \lambda_{1}\right)\left[\mu_{1}\right][e]} \\
& +\Delta t^{2} P_{1} \lambda_{1}\left(1+2 \Delta t P_{1} \lambda_{1}\right)\left[\mu_{1}\right][z] \\
& {\left[R_{31}\right]=\Delta t^{2} P_{2} \lambda_{2}\left[\mu_{2}\right][e]+\Delta t P_{2}\left(2+\Delta t \lambda_{2}\right)\left[\mu_{2}\right][z]} \\
& {\left[R_{32}\right]=\Delta t^{2} P_{1} P_{2} \lambda_{1}\left(\left(2+\Delta t \lambda_{1}\right)\left(2+\Delta t \lambda_{2}\right)-2\right)\left[\mu_{2}\right][z]} \\
& +\Delta t^{2} P_{1} P_{2} \lambda_{1}\left(\Delta t^{2} \lambda_{1} \lambda_{2}-2\right)\left[\mu_{2}\right][e]
\end{aligned}
$$

and

$$
\begin{aligned}
{\left[\mathrm{R}_{33}\right]=} & \Delta t^{2} \mathrm{P}_{2} \lambda_{2}\left(1-4 \mathrm{P}_{2}-2 \Delta t \mathrm{P}_{2} \lambda_{2}\right]\left[\mu_{2}\right][\mathrm{e}] \\
& +\Delta t^{2} \mathrm{P}_{2} \lambda_{2}\left(1+2 \Delta t \mathrm{P}_{2} \lambda_{2}\right)\left[\mu_{2}\right][\mathrm{z}]
\end{aligned}
$$

It can be verified by substitution that equations (III. 2) may be written in the form of (2.36). 


\section{APPENDIX IV \\ Description of Computer Codes}

4. 1 Input Specifications for $\operatorname{MITIM}-\mathrm{E}(2,0)$ and $\operatorname{MITIM}-E(1,1)$

CARD I (5D12.5)

HT - Size of time step (sec.)

HX - Mesh spacing (cm.)

BETOT - Sum of effective delayed neutron fractions

TPRINT - Time at which flux is to be reconstructed and printed

TSTOP - Time at which calculation is terminated

CARD 2 (1015)

NTF - Number of trial functions

NG - Number of neutron groups

NDG - Number of delayed neutron groups

NMP - Number of mesh points

NR - Number of regions

$(\operatorname{NMPR}(\mathrm{I}), \mathrm{I}=1, \mathrm{NR})$ - Number of mesh points in each region

CARD 3 (4D20.10)

$((\operatorname{VSIGF}(\mathrm{J}, \mathrm{K}), \mathrm{K}=1, \mathrm{NR}), \mathrm{J}=1, \mathrm{NG})-v \Sigma_{\mathrm{f}}$ for each group in each region 
CARD 4 (4D20.10)

$(((\operatorname{SIGSR}(J, K, I), I=1, N R), K=1, N G), J=1, N G)-\Sigma_{S_{J}-K}$ for all groups in each region

CARD 5 (4D20.10)

$((\operatorname{SIGTR}(J, K), K=1, N R), J=1, N G)-\Sigma_{T}$, the total absorption cross section, for each group in each region

CARD $6 \quad(4 D 20.10)$

$((D 1(J, K), K=1, N R), J=1, N G)-D$, the diffusion constant, for each group in each region

CARD 7 (4D20.10)

$(\operatorname{BET}(I), I=1, N D G)-\beta_{i}$ for each delayed group

CARD 8 (4D20.10)

$(\operatorname{CHIP}(I), I=1, N G)-\chi_{p}$ for each neutron group

CARD 9 (4D20.10)

( $(\mathrm{CHID}(\mathrm{I}, \mathrm{JJ}, \mathrm{J}=1, \mathrm{NG}), \mathrm{I}=1, \mathrm{NDG})-\chi_{\mathrm{d}}$, the fraction of neuirons produced in each group from decay in each delayed group

CARD $10 \quad(4 D 20.10)$

$(V(I), I=1, N G)-v_{g}$, the neutron velocity for each group 
CARD 11 (4D20,10)

( $\operatorname{LAM}(\mathrm{I}), I=1, N D G)-\lambda_{i}$, the decay constant, for each delayed group Repeat Cards 12 and 13 for each trial function

Repeat Cards 12 and 13 for each neutron group

CARD 12 (6D12.5)

$(\mathrm{PHI}(\mathrm{I}, \mathrm{J}), \mathrm{J}=1, \mathrm{NMP})-\mathrm{V}$ alue of $\mathrm{I}^{\text {th }}$ trial function of each mesh point

CARD 13 (6D12.5)

(WPHI(I, J), J = 1, NMP) - Value of $I^{\text {th }}$ weighting function at each mesh point

CARD $14 \quad(15,2 \mathrm{D} 12.5)$

ITP - index to perturbation

$1=$ step insertion

2 = ramp insertion

Templ - time at which reactivity insertion changes and new time zone begins

Temp2 - time oven which ramp insertion is added

CARD 15 (4D20. 10)

((ALPHA(J,K), K=I, NR), J = 1, NG)) - for ramp insertion, the total change in $\Sigma_{t}$ for each group in each region (leave blank if $\operatorname{ITP}=1$ ) 
CARD $16 \quad$ (4D20.10)

(( $\operatorname{SIGTR}(J, K), K=1, N R), J=1, N G)$ - for step insertion, the new. values of $\Sigma_{T}$ after insertion for each group in each region (omit if $\operatorname{ITP}=2$ ) 
4.2 Code listings for MITIM-E $(2,0)$ and MITIM-E' $(1,1)$ (provided only in first six copies) 